\title{
Cultural Evolution of Genetic Heritability
}

\author{
Ryutaro Uchiyama ${ }^{1 *}$, Rachel Spicer ${ }^{1}$, Michael Muthukrishna ${ }^{1}$
}

1. Department of Psychological and Behavioural Science, London School of Economics and Political Science, Houghton Street, London WC2A 2AE, UK.

* Corresponding author RU: r.uchiyama@1se.ac.uk; https://www.uchiyamaryutaro.com

RS: $\underline{\text { r.a.spicer@lse.ac.uk; } \text { http://www.lse.ac.uk/PBS/People/Rachel-Spicer }}$

MM: m.muthukrishna@1se.ac.uk; https://michael.muthukrishna.com/

\section{SHORT ABSTRACT}

We reconcile behavioral genetics and cultural evolution under a dual inheritance framework. This approach predicts how social factors-such as rates of cultural innovation and diffusion-affect estimates of heritability. Cultural evolution enriches our understanding of the causal relationship between genes and phenotypes; genetic effects are often confounded with cumulative culture. Modeling the effects of cultural dynamics on genetic effects moves us toward generalizability across societies and across time, predicting how heritability should differ between societies, within societies, and over the life course. A cultural evolutionary behavioral genetic approach cuts through the nature-nurture debate and helps resolve controversies such as IQ. 


\section{LONG ABSTRACT}

Behavioral genetics and cultural evolution have both revolutionized our understanding of human behavior-largely independent of each other. Here we reconcile these two fields under a dual inheritance framework, offering a more nuanced understanding of the interaction between genes and culture. Going beyond typical analyses of gene-environment interactions, we describe the cultural dynamics that shape these interactions by shaping the environment and population structure. A cultural evolutionary approach can explain, for example, how factors such as rates of innovation and diffusion, density of cultural sub-groups, and tolerance for behavioral diversity impact heritability estimates, thus yielding predictions for different social contexts. Moreover, when cumulative culture functionally overlaps with genes, genetic effects become masked, unmasked, or even reversed, and the causal effects of an identified gene become confounded with features of the cultural environment. The manner of confounding is specific to a particular society at a particular time, but a WEIRD (Western, educated, industrialized, rich, democratic) sampling problem obscures this boundedness. Cultural evolutionary dynamics are typically missing from models of gene-to-phenotype causality, hindering generalizability of genetic effects across societies and across time. We lay out a reconciled framework and use it to predict the ways in which heritability should differ between societies, between socioeconomic levels and other groupings within some societies but not others, and over the life course. An integrated cultural evolutionary behavioral genetic approach cuts through the nature-nurture debate and helps resolve controversies in topics such as IQ.

\section{INTRODUCTION}

Business is booming in behavioral genetics. We're in the midst of a genome-wide association gold rush (Visscher et al., 2017). The availability of powerful computers and sequenced DNA of millions of people has led to an industrious search for single nucleotide polymorphisms (SNPs) that correlate with a variety of psychological and behavioral traits (Horwitz et al. 2019; Harden and Koellinger 2020; Mills and Tropf 2020). These range from memory capacity 
(Papassotiropoulos et al. 2011), cognitive ability (Coleman et al. 2019) and educational attainment (Lee et al. 2018) to moral attitudes (Brandt and Wetherell 2012), political orientation (Hatemi et al. 2011), temporal discounting (Sanchez-Roige et al. 2018), socio-economic status (Hill et al. 2016), temperament (Zwir et al. 2018), and happiness (Wingo et al. 2017). The significance threshold for discovering correlations is high (a typical threshold being $p<5 \times 10^{-8}$; Fadista et al., 2016) and there are claims that the curse of reverse causality has been lifted. As Plomin and von Stumm (2018) put it, genome-wide polygenic scores "are an exception to the rule that correlations do not imply causation in the sense that there can be no backward causation... nothing in our brains, behavior or environment changes inherited differences in our DNA sequence.”

The last two decades have also seen a parallel revolution in cultural psychology and cultural evolution that has identified significant cultural variation in our psychology and behavior (Henrich, Heine, and Norenzayan 2010; Nisbett 2003; Muthukrishna et al. 2020; Henrich 2016; Muthukrishna and Henrich 2019; Gelfand 2018). These range from fairness and prosocial norms (Henrich, Heine, and Norenzayan 2010; Schulz et al. 2019) and attribution of blame (Barrett et al. 2016) to perceptual style (Kitayama et al. 2003), susceptibility to visual illusions (Henrich, Heine, and Norenzayan 2010), visual perception more broadly (Lupyan et al. 2020), numeric chunking (Domahs et al. 2010), interpretation of linear and logarithmic numeric scales (Dehaene et al. 2008), neural correlates of reading (Bolger, Perfetti, and Schneider 2005; Tan et al. 2005), event segmentation (Swallow and Wang 2020), memory (Amici et al. 2019; Guida et al. 2018; Wang 2021), spatial cognition (Majid et al. 2004), motor development (Karasik et al. 2015), folkbiology (Medin and Atran 2004; Waxman, Medin, and Ross 2007), and personality (Smaldino et al. 2019; Gurven et al. 2013). Cultural evolution is part of a broader theoretical frameworkdual inheritance theory (Cavalli-Sforza and Feldman 1981; Boyd and Richerson 1985) ${ }^{1}$ — that incorporates genes, environment, culture, and learning to offer an explanatory and predictive framework for human psychology and behavior (Muthukrishna and Henrich 2019). This body of research suggests that humans not only have a genetic inheritance from their parents, as do all

\footnotetext{
${ }^{1}$ Research within this framework also falls under culture-gene coevolutionary theory and the extended evolutionary synthesis (Laland et al. 2015).
} 
animals, but also a substantial cumulative cultural inheritance from their societies, well beyond any culture found in other primates, birds, and whales (Dean et al. 2014; although also see Whiten 2019). Genes, culture, and the environment have often co-evolved, shaping our species (Henrich 2016; Laland 2018).

The revolutions in behavioral genetics and cultural evolution have occurred largely independently of each other. Some attempts have been made to integrate the fields (e.g., Laland, Odling-Smee, and Myles 2010; Creanza, Kolodny, and Feldman 2017; Feldman and Ramachandran 2018; Cavalli-Sforza and Feldman 1973; Cavalli-Sforza et al. 1982), with these efforts typically being launched by researchers in cultural evolution. As a result, cultural evolution has incorporated some aspects of behavioral genetics. Behavioral genetics in turn has been largely agnostic with respect to cultural evolution, which is perhaps understandable given the focus, size, and historical trajectory of the field. However, given the extensiveness of the cultural and culturally-shaped environment, cultural evolution offers an important but typically missing complement to otherwise insightful methodological and empirical analyses within behavioral genetics (e.g., Brumpton et al. 2020; Young et al. 2019; Turkheimer, Pettersson, and Horn 2014).

The effect of culture in behavioral genetics is typically incorporated into a broad environmental term that is partitioned into between- and within-family variance components. While behavioral genetic research implicitly or explicitly incorporates an understanding of the way in which genetic evolution shapes genotypes, the environment - cultural or otherwise-is assumed to be exogenous or at best shaped by genes (Plomin, DeFries, and Loehlin 1977; Rutter 2007). The environment is treated as given, while the genome is at least implicitly understood through the lens of selection and function. But just as genetic evolution offers a systematic framework for understanding how distributions of alleles change over time, cultural evolution offers a framework for understanding how distributions of cultural traits change over time; both evolving in response to ecological, demographic, and social factors. Thus, an understanding of cultural change can provide insights into the structure and dynamics of the environmental component of phenotypic variation as well as their interaction with genes. Statistical and theoretical models of gene-environment interactions and correlations are well-trod territory (Purcell 2002; Plomin, DeFries, and Loehlin 1977; Rutter 
2007), but these models do not capture the cultural evolutionary dynamics of environmental change nor the coevolutionary dynamics of gene-culture interactions and correlations.

Humans have long been evolving in ways that deeply intertwine genes and culture: a prominent example is that we have jaws too weak and guts too short for a world without controlled fire and cooked food (Wrangham 2017; Aiello and Wheeler 1995); we lack genes for fire-making or cooking, instead relying on culture to compensate. This kind of coevolutionary history renders some aspects of gene-culture or gene-environment interactions puzzling when taken outside of a dual inheritance framework. Such insights help with interpretation of data. For example, it is well understood and also statistically obvious that reducing environmental variation will increase heritability scores (Stoolmiller 1999). What's less obvious is the way in which culture can either mask or unmask genetic variation, and the way in which cultural diffusion and innovation can increase or decrease heritability. It's similarly not obvious how to define a single society for the purposes of measuring heritability, without being able to identify cultural cleavages that can lead to Scarr-Rowe type effects (see Section 4.1; Turkheimer et al. 2003; Tucker-Drob and Bates 2016). Our goal is to offer a path to reconciliation between behavioral genetics and cultural evolution with new or complementary interpretations for various puzzles, such as differences in heritability between and within populations, differences in heritability across development, and the Flynn effect. In doing so, we hope to instigate a discussion that nuances common interpretations of the nature and nurture of human behavior.

\section{INTERPRETING HERITABILITY}

Heritability is an important metric in behavioral genetics. In its standard formulation, heritability refers to the proportion of phenotypic variance for some trait that is explained by genetic variance. Much has been written about the misunderstandings and pitfalls that commonly occur when interpreting heritability (e.g., Lewontin 1974; Vitzthum 2003; Visscher, Hill, and Wray 2008; Haworth and Davis 2014), and we will not recapitulate these arguments here except when necessary. Suffice to say, heritability is sometimes incorrectly treated as an index of the genetic basis of a trait, and hence as a measure of the relative contribution of nature vis-à-vis nurture. 
Cultural evolutionary theory can contribute to our understanding of the interpretation of heritability by describing the processes of cultural transmission and cultural change. In standard treatments of heritability, phenotypic variance is partitioned into a component explained by genetic variance and a component explained by environmental variance. We will further conceptually partition this environmental component into a sub-component whose distribution is shaped by cultural transmission (the cultural environment or culture) and another whose distribution is not (the ecological environment or ecology). For the purposes of exposition, we will treat these as separable, though of course this dichotomization is merely an approximation as any sharp separation between the two is in reality implausible (Laland et al. 2015).

\subsection{An example: skin pigmentation and UV}

Before we unfold the complexities of interpreting the cultural evolution of genetic heritability, let's begin with a simple illustration that introduces some basic concepts in both behavioral genetics and cultural evolution: the heritability of cancers associated with skin pigmentation. Genes affect the level of skin pigmentation and propensity for tanning instead of burning (Crawford et al. 2017). These are ancestral adaptations to levels of UV radiation at different latitudes (Barsh 2003; Sturm and Duffy 2012). Darker pigmentation protects against high levels of UV radiation, such as near the equator. Lighter pigmentation enables vitamin D synthesis in low levels of $U V$ radiation, such as at Northern latitudes (Jablonski and Chaplin 2010; 2017). It is important to get the correct amount of UV radiation - too much causes skin cancer, but too little causes vitamin D deficiency, which is associated with other health problems (Garland et al. 2006; Edlich et al. 2009).

Worldwide migration has led to people with skin pigmentation mismatched to the level of UV radiation: Australians with European ancestry have higher rates of skin cancer than Australian Aboriginals and other non-European populations (Australian Institute of Health and Welfare 2016), and conversely, Europeans with African and South Asian ancestry have higher rates of vitamin D deficiency and associated afflictions (Cashman et al. 2016; Spiro and Buttriss 2014). A gene by environment interaction $(\mathrm{G} \times \mathrm{E})$ approach could measure how these mismatches affect the 
heritability of skin cancer or Vitamin D concentrations, but not how we should predict these heritability estimates to change over time through cultural evolution, specifically through diffusion and innovation. Through cultural evolution, non-genetic adaptations evolve to compensate for genetic mismatches: fairer Australians wear sunscreen, a hat, and covered clothing (Montague, Borland, and Sinclair 2001) ${ }^{2}$, while darker Europeans consume vitamin D supplements and vitamin D-rich or fortified foods (Spiro and Buttriss 2014).

In this example, the challenges to measuring and interpreting heritability and understanding GWAS results are perhaps more obvious than for many psychological traits. The heritability of skin cancer, for example, should be highest when there is more diversity of skin pigments (genes), more homogeneity of cultural practices (culture), and high UV radiation (ecology) ${ }^{3}$; see Figure 1. While ecology and genes may change to some degree (e.g. smaller hole in the ozone layer and immigration, respectively), cultural change can be particularly fast and potent-greater uptake of anti-skin cancer practices and technologies or new medical interventions for treating cancer. In some cases, cultural evolution is broadly predictable due to directionality - few are working on ways to increase rates of skin cancer. Of course, there may be other forces that work in the opposite direction, such as a tan becoming associated with attractiveness ${ }^{4}$. Here it is easier to see that heritability is a function not only of genes, traits, and ecology, but also of a cultural environment that is evolving according to dynamics that can be understood. The environment of the genome is therefore not an inert backdrop against which genes should be evaluated, but rather, a moving reference frame that rapidly evolves in relation to both genes and ecology.

\footnotetext{
${ }^{2}$ Australia's Slip! Slop! Slap! campaign encourages practices to reduce UV radiation exposure: "slip on a shirt, slop on sunscreen and slap on a hat". More recently, it has been followed by the SunSmart program, which expanded upon the original message to further decrease exposure: "seek shade or shelter, and slide on sunglasses".

${ }^{3}$ An analogous case can be made for Vitamin D deficiency, which should be highest under the same circumstances, except that instead of high UV, it should be low UV levels that most reveal the genetic effect.

${ }^{4}$ Many potential forces may contribute to this: prestige bias creates trends—historically, prior to the 1920s, tanning was associated with working in a field, but after Coco Chanel was sunburnt whilst holidaying in the French Riviera, her fans are said to have copied her tan; success bias as tanning is associated with more time for outdoor leisure; or simply some version of runaway cultural selection (Boyd and Richerson 1985).
} 


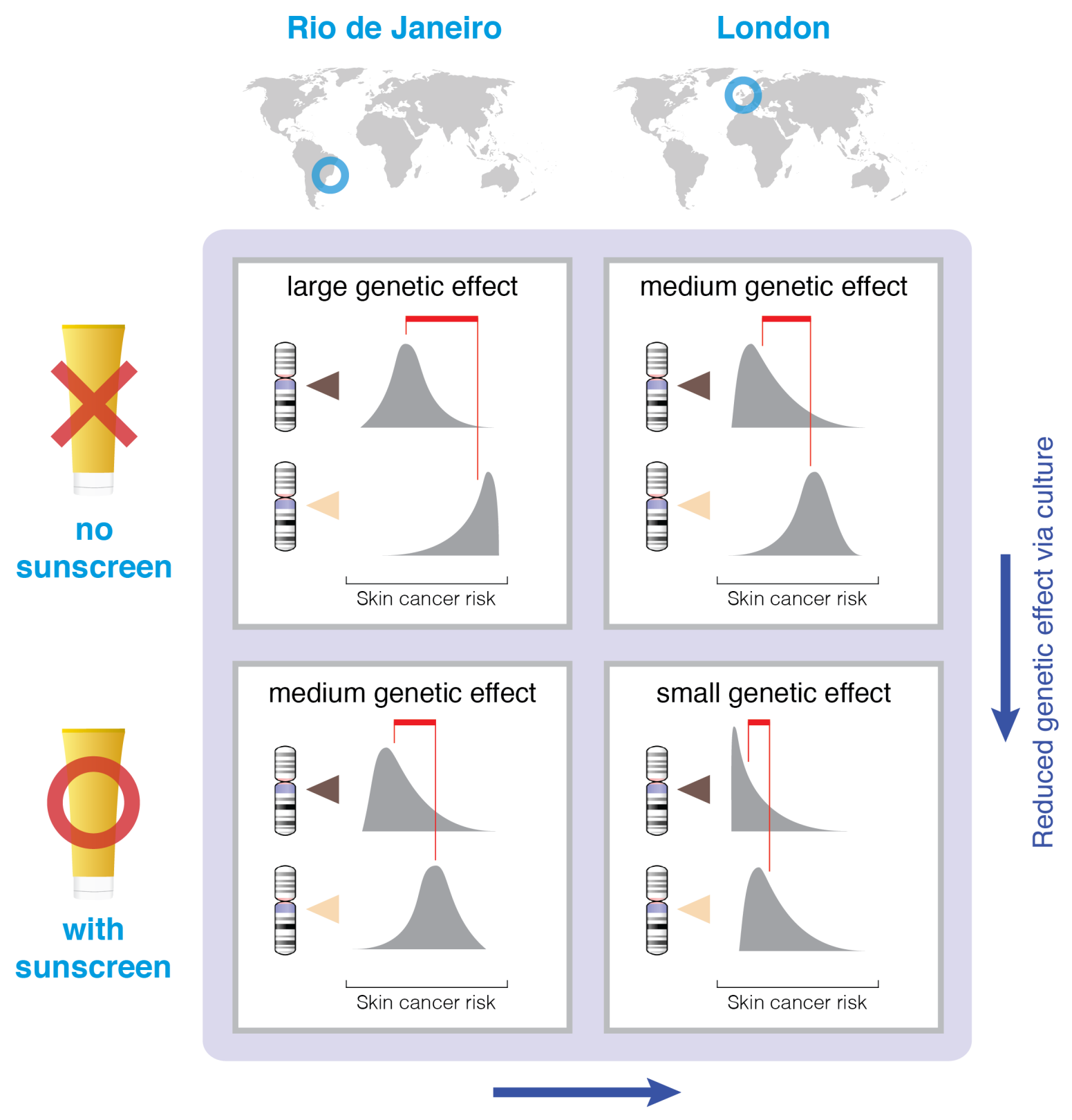

Reduced genetic effect via ecology

Figure 1: An illustration of the effect of sunscreen and geographic location on the effect size of a skin pigmentation gene with respect to skin cancer risk. The largest genetic effect should be found in societies that lack sunscreen and reside in locations with high levels of UV radiation (top-left square). Genetic effects should be reduced with either the introduction of sunscreen or residence in a lower-UV environment, both factors that mask the effect of skin pigmentation (bottom-left and top-right squares, respectively). The smallest effect should be found in societies that have both low UV and sunscreen (bottom-right square). Each cell represents a hypothetical scenario - if Rio and London did or did not have sunscreen. Chromosomes with dark indicators represent genes for strong pigmentation, and those with light indicators represent genes for light pigmentation. Gray distribution represents population distributions for skin cancer risk, and red lines point to the mean of each distribution. 
This example helps us understand four key points. The first of these is well understood by behavioral geneticists and the second is sometimes noted, but the third and fourth points are typically absent from these discussions due to the disconnect between behavioral genetics and cultural evolution. First, there is no overarching, one-quantity heritability of a trait to be discovered. There is no fixed answer to the question, "What is the heritability of skin cancer?". Second, this answer will depend not only on ecology, but also on culture and specifically on cultural diffusion and innovation-both of which can rapidly change and therefore rapidly change heritability estimates. Third, the diffusion and innovation are broadly directional ${ }^{5}$. Cultural diffusion of sunscreen, clothing, shade and sunglasses, and cultural innovation toward more effective screening and treatment of melanomas all work to reduce heritability estimates. In these cases, we expect a reduction in heritability due to the masking effect of the cultural trait. Were any of these an example of culture unmasking genetic effects, such as tanning salons that induce differential risk according to skin pigmentation level, we would have predicted an increase in heritability. Insofar as a preference for avoiding some outcome outweighs other forces that tend to bring it forth, there will be a directional trend over time, similarly to directional selection in genetic evolution (Byars et al. 2010; Sanjak et al. 2018). Fourth, we might also expect the cultural response to be stronger where the ecological and cultural selection pressures are stronger-skin cancer mitigation in Australia but Vitamin D supplementation in northern Europe. Heritability or changes in heritability of these cases have not been tested to our knowledge, but the predictions are clear.

Like heritability, the question "Which SNPs are associated with skin cancer?" is similarly culturally dependent. In societies where sunscreen use is common, we expect SNPs associated with skin pigmentation to be less predictive of skin cancer compared to societies where this is not the case. Similarly, we would expect SNPs associated with antioxidant metabolism (Oskina et al. 2014) to

\footnotetext{
${ }^{5}$ When the cultural forces are well understood, this directionality may be analyzable. And of course, not all cultural forces are adaptive. As in genetic evolution, some may be maladaptive (e.g., female genital mutilation; Efferson, Vogt, and Fehr 2020; Howard and Gibson 2017), mismatched (the Western diet causing disease; Cordain et al. 2005), neutral, or somewhere between these.
} 
be less predictive of skin cancer in societies whose foods are rich in antioxidants-such as in traditional Mediterranean cuisine (Visioli and Galli 2001).

That heritability is affected by the environment is widely understood (Feldman and Ramachandran 2018; Hamer and Sirota 2000; Moore and Shenk 2016; Turkheimer, Pettersson, and Horn 2014; Vitzthum 2003; Tenesa and Haley 2013; Charmantier and Garant 2005; Haworth and Davis 2014). And researchers like Lewontin and Feldman (Lewontin 1970; 1974; Feldman and Lewontin 1975) long ago described the fallacy of extrapolating heritability scores from one population to another. Their argument was made from the standpoint of gene-environment interactions: genetic effects must be understood in the environmental conditions under which the genes are expressed. In this target article we build on this rich body of research to launch a discussion of how the cultural environment changes over time and affects heritability - that is, the cultural evolution of genetic heritability (we schematically capture some of these key ideas in Figure 2). 


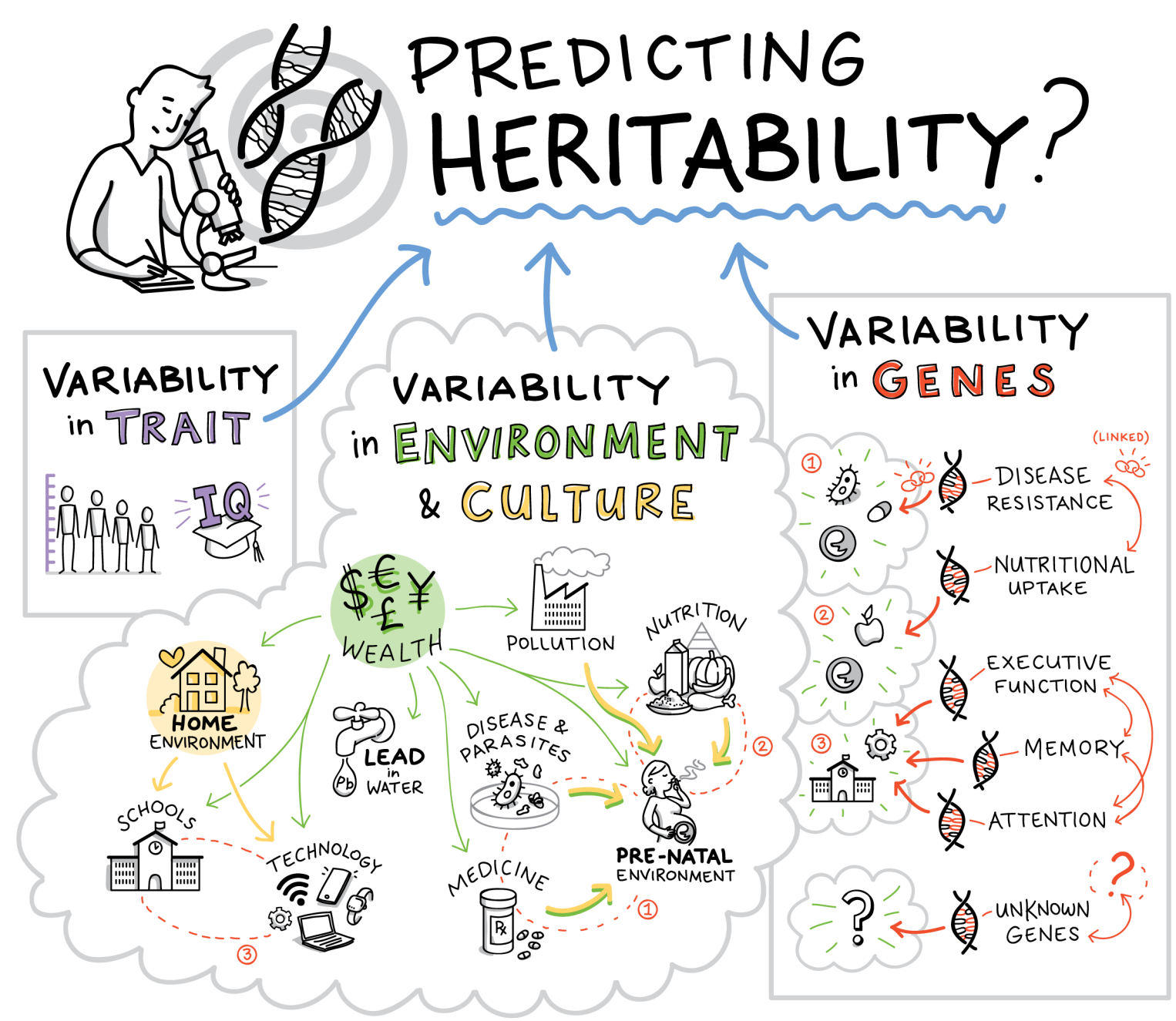

Figure 2: Genetic heritability is a function of variability in the phenotypic trait, variability in the environment, including the cultural environment, and variability in genes. Although heritability is often interpreted as a genetic effect, cultural evolution and diffusion can also systematically shape the variability of environmental variables, and thus heritability. Psychological and behavioral phenotypes are typically the outcome of a complex network of interactions that involve all these factors.

\subsection{Cultural evolution shapes heritability}

Assume that for a given society we were able to collect comprehensive data on genetic effects across all relevant environmental variables that contribute to some trait. This would allow us to exhaustively map out the reaction norms (pattern of phenotypic expression across a range of environments) that specify expected phenotypic outcomes over the full range of extant genetic and environmental variation, thus setting up the conditions for a $\mathrm{G} \times \mathrm{E}$ analysis. But in a species like 
ours, extant environments are not necessarily a meaningful backdrop against which to judge genetic effects. Human environments have already been shaped over deep historical timescales by cumulative cultural evolution-functionally overlapping with genetic evolution (Richerson, Boyd, and Henrich 2010) and can therefore obscure our interpretation of genetic evolution, unless properly accounted for. For humans, the environmental axes of a reaction norm analysis do not simply map out the space of environmental parameters that impact phenotypic outcomes; they rather map out the local and global peaks that have already been climbed by cultural evolution, which correspond to the many solutions to problems that have been discovered and refined over human history. Because cultural evolution enables faster adaptation than genetic evolution, a substantial portion of our adaptations are built into our cultures rather than our genomes.

The human environment is deeply shaped by culture. As heritability is a function of both genetic and environmental variance, cultural evolution carries significant implications for the interpretation of heritability. This idea goes back to the beginnings of the cultural evolutionary paradigm. For example, two founders of the field of cultural evolution, Cavalli-Sforza and Feldman (1972그), modeled the effect of parent-to-child ("vertical") cultural transmission on standard behavioral genetic estimates of genetic effects, showing that vertical transmission should inflate heritability estimates relative to a gene-only model. In contrast, here we focus on a different aspect of the interplay between culture and heritability, namely how oblique and horizontal transmission can impact heritability through processes of broad diffusion across a society that systematically shape phenotypic distributions. We begin this discussion by describing the effects of cultural diffusion and innovation upon heritability, as well as the predictions we can make for differences in heritability between societies.

\subsubsection{Cultural diffusion}

In human societies, mechanisms such as conformist-biased learning (learning from the majority or plurality at a rate above population frequency; Muthukrishna, Morgan, and Henrich 2016; CavalliSforza and Feldman 1981; Boyd and Richerson 1985), payoff biases (e.g. learning from successful others; Cavalli-Sforza and Feldman 1981; Boyd and Richerson 1985), and norm enforcement 
(identifying norms and punishing norm violators; Chudek and Henrich 2011) result in particular behaviors, beliefs, and norms disseminating widely across a society. To the degree that these cultural traits mask the effects of genes (such as in the skin cancer and Vitamin D examples), heritability is reduced. In contrast, to the degree to which these cultural traits unmask or interact positively with genes without masking, heritability is increased.

One example that can illustrate both masking and unmasking through diffusion are genes that support the perception of lexical tone in tonal languages like Cantonese and Yoruba (Dediu and Ladd 2007; Wong et al. 2020). To the degree that tonal languages like Cantonese or Yoruba diffuse in the population (and holding constant other genetic contributions), heritability of language ability would increase in a manner proportional to variation in these genes; to the degree that non-tonal languages like Norwegian or Russian diffuse in the same population, heritability of language ability would decrease. As another example: it is known that fertility is predicted by genes (Zietsch et al. 2014), but Briley, Harden, and Tucker-Drob (2015) report a large rise in the heritability of fertility in the US over mid-20th century, likely due to the increase in the variety of acceptable reproductive choices that was brought about by the diffusion of new social values. In this case, an increase in cultural variance unmasked the effect of genes associated with reproductive behaviors and preferences. Conversely, cultural or policy changes that reduce variation in reproductive practices—such as rigid childbearing norms or a one-child policy—would be expected to mask the genetic effect.

The diffusion of cultural traits is not random and is in fact well studied within cultural evolution and elsewhere (Rogers 2003; Henrich 2001; Muthukrishna and Henrich 2016). One mechanism for cultural diffusion that is important in industrialized societies is formal education. We use this example, because the effect of this particular cultural institution on heritability has been studied. Samuelsson et al. (2008) measured the heritability of reading and spelling test scores. Australian twins demonstrated a narrow-sense heritability of 0.84 in kindergarten and a similar score of 0.80 in Grade 1. In contrast, Swedish and Norwegian twins demonstrated a heritability of only 0.33 in kindergarten, rising to 0.79 in Grade 1. Heritability was at the same level in both the Australian and Scandinavian children in Grade 1, but not in kindergarten. Why? Cultural diffusion of literacy. 
Australian children begin receiving compulsory literacy instruction in kindergarten, while in Scandinavia the kindergarten curriculum emphasizes social, emotional, and aesthetic development-literacy instruction only begins in Grade 1 . Here we see the effect of national curriculum policy differences affecting cultural diffusion of literacy and thus heritability, in a case where the cultural trait interacts with genes. Australian kindergarteners are exposed to standardized environmental input and much of the remaining variation in reading ability is explained by genetic differences, whereas for the Swedish and Norwegian kindergarteners, variation in the amount of reading instruction received at home is much larger than any genetic differences. In line with this interpretation, Samuelsson et al. (2008) show that the boost in heritability among the Scandinavian children was also accompanied by an almost equivalent decrease in phenotypic variance attributed to the common (home) environment, which would include home instruction.

If we were to assess the genetic basis of literacy skill in schoolchildren without accounting for the impact of their particular educational curricula on cultural diffusion and environmental variation, we would be subjecting ourselves to a selection bias, with no idea of the magnitude of this bias. This would distort our understanding of the generalizability of our finding to samples that have undergone different educational curricula, and even more so to those with different levels of educational attainment. Note that even the literacy instruction provided in the home environment is already shaped by cultural evolution, both in terms of the content being transmitted (reading and spelling), and the structures that are transmitting (family organization in Western countries; Schulz et al. 2019; Henrich 2020). In societies that produce literate children, culture impacts the heritability of literacy from the moment that variation in this trait emerges in development, virtually sealing off the possibility of assessing 'baseline' heritability without cultural interference, even at the very start of life (e.g., children born in literate societies are typically surrounded by writing and literate adults). Heritability is a composite measure that captures both genetic and cultural effects, and without knowledge of the cultural context, it is difficult or impossible to judge what is being measured. When we say that the heritability of reading among Scandinavian children jumps up to 0.79 when they enter Grade 1 , this measurement reveals just as much if not more about the disseminative power of modern schooling than it does about the genetic basis of literacy. 
Looking toward a broader social context, several insightful studies have shown that the heritability of educational attainment increases with equality of opportunity. We see evidence for this within countries over time (Heath et al. 1985), within and between countries over time (Engzell and Tropf 2019; de Zeeuw, de Geus, and Boomsma 2015), and within countries following a major policy change (Ujma et al. 2020; Rimfeld et al. 2018; Colodro-Conde et al. 2015). Although there is also some evidence going in the other direction (Silventoinen et al. 2020), the overall pattern appears to be that diffusion of educational opportunity results in genes explaining a relatively greater proportion of variance in educational attainment. These studies serve as robust demonstrations of how heritability can be boosted by changes in social context broadly, and diffusion of particular forms of environmental exposure more specifically. Although these patterns are broadly consistent with our thesis, both the consistent and inconsistent results may obscure cultural structuring, such as the hidden cluster problem and cultural Simpson's paradox, as we discuss in Section 3.

\subsubsection{Cultural innovation}

The human capacity for cumulative cultural evolution (Henrich 2004a; Dean et al. 2014) ensures that in our societies, the diffusion of extant cultural traits goes hand-in-hand with the continual generation of new cultural traits. Whether emerging through serendipitous discovery, iterated trial-and-error, or recombination of ideas, some portion of new technologies and behaviors manages to spread across a society, displacing other competing variants. The dynamics of innovation are a significant component of the cultural evolutionary framework (Muthukrishna and Henrich 2016; Henrich 2004a).

Cultural evolution predicts that societies will vary in their rate and type of cultural innovation (e.g. number of patents and incremental vs. revolutionary invention) due to differences in, for example, the size and interconnectedness of their social networks (sociality), effectiveness of cultural transmission (e.g. education), and tolerance for diversity; for review see Muthukrishna and Henrich (2016). Tolerance for diversity can drive differences in heterogeneity. Some societies such 
as Pakistan and Indonesia maintain relatively low levels of cultural heterogeneity, whereas others such as Brazil and Australia maintain relatively high levels of heterogeneity. One metric that can serve as a proxy for this tolerance is cultural tightness/looseness, which represents the degree to which societies tolerate deviation from social norms, and is expected to correspond to their rate of innovation (Gelfand, Nishii, and Raver 2006; Gelfand et al. 2011; Gelfand 2018). Societies that are more tolerant of cultural variation allow for more individual-level exploration, and if the best of these cultural mutants can be selectively incorporated into the mainstream, those societies will tend to undergo higher rates of increase in cultural complexity (Henrich 2004a). Cultural variation is the engine of cultural change. Aside from variables like cultural looseness, policies such as social safety nets and forgiving bankruptcy laws can also create the incentive structure for promoting exploration and innovation (Muthukrishna and Henrich 2016).

The initial spread of an innovation will be tied to an increase in environmental variance if that innovation is disrupting an earlier, relatively homogenous state. To the degree that innovation is masking genes, heritability will begin to decrease. To the degree that innovation is unmasking genes, heritability will begin to increase.

\subsubsection{Predicting differences in heritability across societies}

Over long historical durations, cultural evolution tends toward a compressive environmental effect, as ecological problems are solved and more favorable environmental conditions spread. But on shorter timescales, diffusion and innovation have opposing effects on environmental variance. Although indoor plumbing, antibiotics, and formal education have diffused to the point of being ubiquitous in the Western world, new innovations are also constantly emerging. During their early spread, such innovations increase environmental variance and between-group differences, thereby

bringing forth new forms of diversity and inequality. But as these new traits diffuse further, some of them become gradually established within the cultural corpus, reducing environmental variation through homogenization. The dynamics of diffusion and innovation thus differ in their effects, although the magnitude and direction of these effects also depend upon whether the trait in 
question masks or unmasks genetic variation. Moreover, across societies, the balance between diffusion and innovation differs as well.

If we imagine a society in which innovations are rapidly diffused across the whole society shortly after they emerge, the society will have a low level of cultural variation at most times. This is a society in which a high rate of diffusion quickly overpowers the environmental variance-increasing potential of cultural innovation. Now imagine a contrasting society in which innovations take a much longer time to diffuse. Perhaps the society is more culturally clustered with less interaction between individuals in different subgroups, or perhaps there is a subset of individuals who are wellconnected with the inventor and become early adopters but the novel trait diffuses only gradually beyond this inner circle. This would be a culturally unequal society that is characterized by high environmental variability at any given point in time. This kind of stunted diffusion may suggest cultural clustering, with relatively dense connections within subgroups and relatively sparse connections between subgroups. In such societies, even highly useful forms of cultural knowledge may not easily permeate social barriers, and the waves of environmental change are correspondingly 'loose'. These barriers are not necessarily ethnic boundaries: they could be organized around class, wealth, occupation, political alignment, religion, or incidental geographic layout. A society may be clustered for reasons that stem from within the society itself, or it could simply be a matter of nominal mismatch between the political boundaries that we use to refer to a society and the actual organization of cultural groups. Greater differential clustering can lead to a cultural Simpson's paradox (discussed in Section 3.4).

We can derive testable hypotheses from these dynamics: culturally homogeneous societies will have higher heritability for culturally transmissible phenotypic traits ${ }^{6}$ compared to culturally diverse or clustered societies. We model this prediction in the Appendix. One way to measure cultural diversity would be to use cultural tightness/looseness as a proxy (Gelfand, Nishii, and Raver 2006; Gelfand et al. 2011). One way to measure cultural clustering would be to use the cultural fixation

\footnotetext{
${ }^{6}$ Culturally transmissible traits are those whose distribution can be shifted by cultural learning (e.g. many psychological and behavioral traits).
} 
index $\left(C F_{S T}\right)$ as a measure of cultural distance between groups within a society (Muthukrishna et al. 2020)—we discuss this in more detail in Section 3.2. Whatever the measure, homogeneity of cultural traits should be associated with higher heritability on average, but with respect to specific traits, the relationship between homogeneity and heritability will also depend upon the genetic masking or unmasking effect of the trait.

For cultural traits that neither mask nor unmask genetic effects, heritability will generally be higher in culturally homogeneous societies than in culturally diverse societies due to the reduced environmental variance that is a consequence of behavioral uniformity, as described above. For cultural traits that unmask genes, heritability will be higher overall compared to traits that neither mask nor unmask, and on average higher in homogeneous societies than in diverse societies. For cultural traits that mask genes, heritability will be lower overall compared to traits that neither mask nor unmask, but whether it is higher in homogeneous or diverse societies will depend upon which has the stronger effect: genetic masking or the reduction in environmental variance accompanying homogeneity. If the effect of genetic masking is stronger, heritability will be lower in culturally homogeneous societies than in culturally diverse societies; if the reduction in environmental variance is stronger, heritability will be higher in homogeneous societies than in diverse societies.

Thus heritability estimates reflect cultural evolutionary dynamics. Researchers such as Harden (2021) have highlighted how heritability can be used as a tool for measuring useful characteristics across environments such as cross-national differences in social opportunity. These interpretations are consilient with our approach, but are limited by treating the environment as exogenous rather than modeling its dynamics and interactions. Such complexities in the role of the cultural environment have historically been obscured, due in part to a number of methodological and epistemological problems that encumber standard behavioral genetic analysis. 


\section{PROBLEMS THAT OBSCURE THE EFFECT OF CULTURE}

The effects of cultural evolution on our understanding of heritability are complex, but we hope that at least the significance of this relationship has now become more obvious. At the very least, we hope this target article will spark a vibrant discussion of the role of cultural evolution in behavioral genetics. There are several features of behavioral genetic methodology that tend to obscure the effect of culture and cultural evolution. Here we will discuss three of these problemsthe WEIRD sampling problem, the hidden cluster problem, and the causal locus problem-and then describe a cultural Simpson's paradox that emerges at their junction. Various aspects of these problems have been discussed in prior literature, but we will focus on how the problems specifically interact with the detection and interpretation of effects stemming from cultural evolution. Clarity regarding these issues will be a first step toward integrating the cultural evolutionary framework with behavioral genetics.

\subsection{WEIRD sampling problem}

Behavioral genetics suffers from its own variant of the Western, educated, industrialized, rich, democratic (WEIRD) people problem, which was originally raised in the field of experimental psychology (Henrich, Heine, and Norenzayan 2010; Apicella, Norenzayan, and Henrich 2020). The WEIRD people problem refers to the vast over-representation in published studies of individuals from developed Western countries, who are similar in their cultural history, social values, and standards of living. Just as behavioral experimental samples are psychologically WEIRD, behavioral genetic samples are both genetically and culturally WEIRD. This results in a WEIRD sampling problem that limits the variation required to make sense of genetic effects. This problem involves both genetic and cultural restriction of range, and we will discuss each in turn.

\subsubsection{WEIRD genetics}

A comprehensive meta-analysis that claims to contain essentially all twin studies published between 1958 to 2012 (Polderman et al. 2015) reveals that 94\% of sampled twin pairs were from Western populations. The United States, United Kingdom, and Australia alone accounted for 
almost 60\%, and Nordic countries accounted for another 25\%. Of the non-Western countries (6\%), two thirds (4\%) are from northeast Asia-specifically, China, Japan, South Korea, and Taiwan, countries that are not Western, but have most of the remaining letters of the WEIRD acronym. The remainder of the world, representing the vast majority of the human population, accounts for only $2 \%$ of the dataset.

GWAS too suffers from a myopic focus on WEIRD genomes (Need and Goldstein 2009; Popejoy and Fullerton 2016; Sirugo, Williams, and Tishkoff 2019). As of 2017, 88\% of samples in GWAS were of European ancestry (Mills and Rahal 2019)7. Paralleling the twin studies data, 72\% of participants were recruited from just three countries-US, UK and Iceland-with nearly $20 \%$ of the remainder being recruited from Japan, China and South Korea.

Polygenic scores do not translate well across ancestry groups (Bitarello and Mathieson 2020; Guo et al. 2021; Curtis 2018; Kim et al. 2018; Martin et al. 2017; 2019). For example, European ancestry-derived polygenic scores have only $42 \%$ of the effect size in African ancestry samples (Duncan et al. 2019). From a cultural evolutionary perspective, this is not unexpected given the cultural environment, coevolution between culture and genes, and cultural differences between populations.

Polygenic scores are also highly sensitive to inadequately controlled population stratification (Berg et al. 2019; Sohail et al. 2019; Morris et al. 2020). Even within a single ancestry group, the predictive accuracy of polygenic scores can be dependent on age, sex and socio-economic status (Mostafavi et al. 2020) — this too, from a cultural evolutionary perspective, is not unexpected given the cultural variation that exists within a population (Muthukrishna et al. 2020; Muthukrishna and Henrich 2019). Similarly, the SNPs that contribute to the variance of a trait are different in different populations (Pemberton et al. 2018; Gurdasani et al. 2019; Akiyama et al. 2019; Rotimi et al. 2017) and it is difficult to disentangle the genetic, environmental and cultural contribution

\footnotetext{
${ }^{7}$ An improvement from 2009 when 96\% of GWAS participants were of European ancestry (Need and Goldstein 2009)!
} 
to differing polygenic scores between populations (Rosenberg et al. 2019). Recent projects have aimed to capture a greater degree of global human genetic diversity (e.g. Simons Genome Diversity Project, Mallick et al. 2016; the exome analysis of Lek et al. 2016; and the GenomeAsia project, Wall et al. 2019), but we are far from proportionately representing the genetic diversity of the global population.

\subsubsection{WEIRD culture}

When we restrict the scope of genetic samples, the cultural environment against which genetic effects are evaluated also becomes skewed, and this greatly reduces the interpretability of genetic effects. Due to a combination of cultural group dynamics (Chudek and Henrich 2011; Henrich 2004b; Richerson et al. 2016) and cumulative cultural evolution (Henrich 2004a; Dean et al. 2014), the human species is characterized by large amounts of cultural and hence environmental variation between societies, which exceeds genetic variation by orders of magnitude (Bell, Richerson, and McElreath 2009). The WEIRD countries that are over-represented in genetic samples are clustered together along multiple cultural dimensions (Hofstede 2001; Inglehart and Welzel 2005; Muthukrishna et al. 2020), and are perhaps an extreme unrepresentative outlier on many psychological and behavioral measures, with these countries registering the highest scores for traits like individualism, analytical thinking, and prosociality toward strangers, and the lowest scores on opposite constructs such as collectivism, holistic thinking, and prosociality toward relatives but not strangers (Henrich, Heine, and Norenzayan 2010; Muthukrishna et al. 2020; Schulz et al. 2019; Henrich 2020).

WEIRD societies are also roughly aligned on basic environmental factors like technology, civil infrastructure, healthcare, schooling, and transportation, with typically advanced levels in each of these. Within each of these countries as well, inequality is low compared to non-WEIRD countries, as can be inferred from the comparatively low Gini coefficients of Western countries, with the US being somewhat of an unequal outlier (Selita and Kovas 2019). 
Just as this restricted cultural range limits our understanding of human psychology (Henrich, Heine, and Norenzayan 2010), it also limits the inferences that can be made about human genetics. Behavioral geneticists understand that genes can have different effects under different environmental conditions (Assary et al. 2018; Moffitt, Caspi, and Rutter 2005), but there is less appreciation of how those environmental conditions and interactions change through cultural evolution. Cultural evolution can mask or unmask genetic effects, such that gene function becomes confounded with the effect of the cultural environment in a manner that is dependent on the specifics of the underlying dynamics. Cutting through this confound requires a theoretical understanding of the cultural dynamics as well as empirical data on genetic effects across a wide variety of cultural environments. The WEIRD sampling problem therefore harms accurate inference of genetic effects.

\subsection{Hidden cluster problem}

Despite this severe restriction of range among behavioral genetic samples, these samples may also paradoxically be too inclusive. This is due to the presence of cultural clusters that introduce population structure into genetic samples, but which remain hidden to standard behavioral genetic methods. A cultural evolutionary approach can help reveal environmental structure that does not necessarily map onto conventional demographic groupings.

\subsubsection{Cultural clustering}

Whether the method is a twin study or GWAS, behavioral genetic studies typically draw their data from databases such as twin registries or biobanks, whose coverage spans some circumscribed geographic range such as a country or a subnational region. Because these samples trace over political or administrative boundaries, they may not match up with the actual structure of environmental variation, which will often be dependent upon the organization of cultural clustering. Culture generally tends to agglomerate, such that groups of individuals who are bound together by dense links of cultural influence share a substantially greater number of features of their cultural environment than do individuals who belong to different groups (Richerson et al. 2016). 
As has been discussed extensively in the cultural evolutionary literature, this in-group similarity is generated and maintained by processes such as conformist learning (Boyd and Richerson 1985), norm enforcement (Fehr and Fischbacher 2004; Chudek and Henrich 2011), symbolic markers of in-group membership (Boyd and Richerson 1987), and cultural group selection (Henrich 2004b; Richerson et al. 2016). The structure of environmental variation is thus shaped by networks of cultural interaction, and the topology of these networks is assembled over time by cultural group dynamics and cultural evolution.

A highly clustered population is one that can be easily partitioned into multiple subgroups whose members share among each other cultural traits that are substantially different from those found in other subgroups. When we know the underlying social network, network clustering algorithms (Emmons et al. 2016) can help identify these clusters. When we have measures of a range of cultural traits, we can see the effects of clustered cultural influence by measuring the cultural distance between subgroups, for example by using $C F_{S T}$ (Muthukrishna et al. 2020). $C F_{S T}$ applies the 'fixation index' (Cavalli-Sforza, Menozzi, and Piazza 1994) to the World Values Survey of cultural beliefs and behaviors (Inglehart et al. 2014), and can quantify the cultural differentiation between any two groups. When applied to sub-groups within societies, this measure can tell us, for example, the degree to which Singaporean Catholics differ from Singaporean Protestants, thus identifying the degree to which a nation state or other political grouping contains large clustering (note that this is different to diversity—a country may be diverse, but uniformly so, showing no large clustering).

Cultural clusters are typically created by barriers that impede cultural interaction, such as topography (e.g., mountain ranges or bodies of water separating populations) or cultural conflict (e.g., conflicting religious beliefs). Mutually unintelligible languages are an example of a social barrier that impedes cultural and even genetic mixing. Thus, the density of languages within a population will also predict clustering. A topographically fractured country like Papua New Guinea with its 839 often mutually unintelligible languages is likely to be a much more clustered population than an equally populated but less multilingual society like Austria. Populations can also become clustered due to more extrinsic factors. Countries that grew through recent 
immigration, like Canada, are likely to have more clustering than countries that are characterized by relative cultural homogeneity, like Japan (Fearon 2003). Countries whose borders are drawn arbitrarily with respect to the geographic arrangement of cultural groups, for example as a product of past colonial administration (many countries in Africa), are also likely to have high clustering (Michalopoulos and Papaioannou 2020). Note the parallel between the present argument and assortative mating (Schwartz 2013): whereas assortative mating results in phenotypic clustering due to reproduction, cultural transmission results in phenotypic clustering due to segregated learning.

Moreover, cultural clusters may cut through divisions of society that we don't always think of as being 'cultural', such as stratification by social class or wealth. The strength of this stratification regulates the amount of within-stratum versus between-stratum cultural interaction. Although this picture may be complicated by factors such as prestige bias engendering asymmetric influence from upper to lower classes (Henrich and Gil-White 2001), such forms of social hierarchy can create clusters. These examples show that cultural clustering is not necessarily bound by geographic contiguity. Although spatial proximity is a good predictor of cultural influence, cultural influence may also be decoupled from geography, particularly with modern forms of mass media and information technology (Martin and Yurukoglu 2017; Anderson 2006). In section 4.1, we discuss in more detail how cultural clustering may contribute to the phenomenon of differential heritability across socio-economic levels.

In sum, the scope of a genetic sample is often defined by political and administrative boundaries, but these boundaries may be mismatched to the organization of cultural clusters within a society, and the extent of this mismatch will vary greatly across populations. So in addition to measuring cultural variation (diversity) using indices such as cultural looseness, we need to also measure cultural clustering ("segregated diversity") to fully understand the environment. Once we are able to do this, the next intellectual step would be to achieve an understanding of the superimposition of cultural and genetic clusters as well as their interaction. 


\subsubsection{Contrasts between cultural and genetic clustering}

Behavioral geneticists have devoted much effort to the study of genotypic clustering, usually referred to as population stratification or population structure. Populations can become stratified in the presence of genetic variation arising from systematic differences in subpopulation ancestry or from other forms of structured, non-random mating (Brumpton et al. 2020). This kind of genetic clustering can introduce spurious associations between genotypes and traits. As such, researchers continue to develop an array of methods for dealing with this problem (Hellwege et al. 2017; Price et al. 2010).

Genetic clustering and cultural clustering are linked-a well-known example is the close alignment of genes and languages in pre-modern populations (Pagani et al. 2012; Tishkoff et al. 2009; Cavalli-Sforza et al. 1988; Cavalli-Sforza 2001). This gene-language alignment occurs because languages, like genes, have traditionally been transmitted vertically-that is, from parents to children or within the family. This is due to critical periods for language learning that close as early as 6 months for phonology (Kuhl et al. 1992) and age 7 for grammar (Johnson and Newport 1989), due to plasticity in the relevant cortical networks being progressively staunched by "molecular brakes" (Werker and Hensch 2015). Early learning of this kind is primarily dispensed by parents or other immediate caretakers (Kline, Boyd, and Henrich 2013; Hewlett et al. 2011), and it is unsurprising that genes and languages should be largely aligned in reconstructions of population history ${ }^{8}$.

However, beyond early childhood, children become increasingly exposed to other children from different families as well as to non-kin adults, and the range of sources for cultural learning widens accordingly: a two-stage model of cultural learning (Kline, Boyd, and Henrich 2013). Due to transmission through these horizontal (i.e., age-peers) and oblique (i.e., non-parent adults) channels, cultural learning becomes untethered from strict vertical transmission and extends laterally across genetic lineages, similar in structure to horizontal gene transfer that is prevalent in

\footnotetext{
${ }^{8}$ Note that this is not the case for modern multicultural populations that can expose children to languages not spoken by their parents or family during this critical period.
} 
bacteria and archaea (Soucy, Huang, and Gogarten 2015). Like horizontal gene transfer, horizontal cultural transmission is rapid, due to it being unconstrained by the generational cycle of vertical transmission. Oblique and horizontal transmission play a large role in cultural diffusion both within and across extant small-scale societies (Kline, Boyd, and Henrich 2013; Hewlett et al. 2011; Henrich and Henrich 2010), just as they do in large-scale industrialized societies where innovation, knowledge, and practices across domains such as technology, art, education, political systems, and supernatural beliefs commonly diffuse horizontally (Rogers 2003). The importance of oblique and horizontal transmission in human cultural evolution may have been amplified by adaptations such as the timing of weaning relative to brain maturation, which is substantially earlier in our species than would be expected on the basis of our close primate relatives, thereby expediting the child's exposure to cultural models other than parents (Hawkes and Finlay 2018; Finlay and Uchiyama 2020). Indeed, with only vertical transmission, cultural evolution as it is currently understood would not work (Enquist et al. 2010).

Due in part to horizontal transmission, the diffusion of culture can occur much more rapidly than the diffusion of genes, or "demic" diffusion. In historical populations, the speed of demic diffusion has been limited by rates of reproduction and migration (Ammerman and Cavalli-Sforza 1984; Fort 2012). Horizontal transmission is the diffusion of ideas rather than people and has no such limits; cultural clusters are therefore more fluid and malleable than genetic clusters. For example, if archaeological data show cultural commonality between two contemporaneous populations but genomic analyses suggest disjunction, we can often infer that the shared cultural traits between the two groups was due to cultural transmission rather than migration or admixture (e.g., Olalde et al. 2018; Fu et al. 2016).

Cultural clusters are not only more fluid than genetic clusters, they also explain intergroup differences better than genes do. Cultural distances between neighboring countries is an order of magnitude larger than genetic distances (Bell, Richerson, and McElreath 2009). Among smallscale societies in Kenya, pastoral clans are differentiated by cultural traits considerably more than they are by genes, and cooperation among these clans is predicted by their cultural-but not geographic-distance to each other (Handley and Mathew 2020). These findings are consistent 
with the predictions of cultural-group selection (Henrich 2004b; Richerson et al. 2016), particularly in how competition between cultural trait-groups helps explain the evolution of human cooperation (Henrich and Muthukrishna 2021). Cultural-group selection requires large differences between groups of cultural traits relative to differences within groups (Fehr and Fischbacher 2003; Apicella et al. 2012).

Reconciliation between cultural evolution and behavioral genetics requires an update in the way we think about culture. An ethnolinguistic conception of culture that revolves around vertical transmission roughly in alignment with genes is insufficient. For example, linguistic, ethnic, religious, or caste boundaries can create genetic population structure through endogamous marriage practices, whereby individuals marry others who are culturally similar. In the modern world, such assortative mating may be exaggerated as people become more mobile and better connected, making it easier to mate with partners who share cultural traits across many dimensions. Efficiency of assortment may be boosted by cultural matching through dating apps, stretching the tails of the genetic distribution. These are all examples of human culture but they represent only one aspect of it, namely cultural effects that channel mating and thereby regulate genetic clusters. This aspect of culture is what appears as signal in population genetic data and is thus often the focus of attention for geneticists insofar as culture is concerned. This ethnolinguistic conception of culture that reduces culture to its effects on genetic clustering is inadequate for comparing genetic effects to environmental effects, given the many non-reproductive cultural effects that shape human environments and phenotypes. Indeed, an understanding of cultural dynamics and clustering may elucidate novel aspects of genetic population stratification.

Abdellaoui and colleagues (Abdellaoui, Verweij, and Nivard 2021; Abdellaoui et al. 2019) offer evidence for geographic clustering of polygenic scores in the United Kingdom that is statistically independent of genetic ancestry, and explained instead by recent migration. For example, coal mining regions experienced "brain drain" and other forms of trait-conditional departure due to changes in the UK economy over the 20th century. The outcome is detectable in genetic assortment at both the source and destination regions. This is an example of gene-environment correlation, where the environmental factor is a macroeconomic variable with a relatively clear 
geographic distribution. But this kind of genetic sorting is likely to occur also with respect to environmental factors that are shaped by cultural transmission yet not as readily localizable along geography or other conventional social scientific dimensions. Just as we can use genomic methods to reconstruct aspects of cultural history (e.g., Peter, Petkova, and Novembre 2020; Petkova, Novembre, and Stephens 2016; Dai et al. 2020), a research strategy that builds upon cultural transmission may help us reconstruct and even predict the emergence of genetic clusters that coalesce around — and perhaps feed back onto—cultural clustering of human behavior, psychology, and environments.

\subsection{Causal locus problem}

The hidden cluster problem described the implicit complexity that exists within social groupings, which can hinder robust inference unless parsed properly. Below, we discuss the cultural locus problem, which refers to a source of complexity that exists within the space of functional organization in which genes and culture interact. Inference is hindered here when we view culture as an unstructured exogenous variable, when in fact it is a constructive system that accumulates functional adaptations in a directed manner over time.

\subsubsection{Genes that break and genes that make}

The more complex a system, the more ways it can fail. Take the history of lighting: compared to the two ways in which a wood-fueled fire can be extinguished (smothering and exhaustion of fuel), there are 7 known failure modes for a fluorescent bulb and more than 30 for the newer LED bulb (de Groot et al. 2013). A faulty rubber O-ring caused the space shuttle Challenger to explode, and a severed fiber-optic cable knocked out internet access for a large swath of people across India and the Middle East. There is a fundamental asymmetry between the identification of elements that support a system and those that undermine it. A well-functioning system is the product of a design process that has solved many problems and closed many paths that do not work. For such a system, 'something going wrong' can be caused by singular aberrations, but 'going right' or 'going well' are properties of the integrated system as a whole. The notions of success and failure in this context 
therefore point to very different things. For complex functional systems such as machines and organisms, it is easier to identify ways to break the system than ways to explain or improve it.

Gene function can be viewed through this lens. Organisms are the outcomes of complex, emergent interactions involving many genes and their surroundings (Davies 2014), but there are many ways these interactions can go wrong. It is easier to identify deleterious genetic mutations than beneficial mutations, as deleterious mutations are more common. The space of failure is larger than the space of success, making genes that break more detectable than genes that make. For example, a single mutation can cause Mendelian disorders such as cystic fibrosis and Huntington's disease, but no single mutation creates genius. Over 1000 genes have been linked to intelligence (Savage et al. 2018; G. Davies et al. 2018; Lee et al. 2018). Each gene only explains a miniscule fraction of variation in intelligence, and the causal mechanisms are unlikely to be straightforward (Gottlieb 2003; Turkheimer 2000). In contrast to these genes that make, the causal mechanisms behind single gene mutations that cause intellectual disability-e.g. BCL11A (Dias et al. 2016), PHF8 (Bathelt et al. 2016), ZDHHC9 (Schirwani et al. 2018)—are relatively well understood.

This spectrum of localizability ranging from Mendelian to polygenic to "omnigenic" traits (Boyle, Li, and Pritchard 2017) has been discussed extensively, but its interaction with cumulative culture has not sufficiently been appreciated. We have known for a long time that increasing nutrition (Lynn 1990; Stoch et al. 1982), improving schooling (Ceci 1991; Davis 2014; Ritchie and TuckerDrob 2018), and removing parasites (Jardim-Botelho et al. 2008) have positive effects on IQ. None of this is surprising, but it means that in a society where parasite infection is kept under control, we would not notice that parasite status correlates with intelligence, due to a lack of sufficient variation in parasite load. For the same reason, a correlation between lead exposure and IQ (Needleman and Gatsonis 1990; Wasserman et al. 1997) will not be revealed in a society where lead is not a problem. And by corollary, genes that provide protection against malnutrition, parasites, or pollution would only be positively associated with intelligence in environments where these insults occur. In environments where these insults have been removed, the same genes would not be associated with intelligence, and can even be deleterious, as in the well-known example of sickle cell trait (Elguero et al. 2015). Similarly, alleles that protect against parasite infection (Carter 
2013) or lead poisoning (Onalaja and Claudio 2000) will be predictive of IQ only if the environmental risk factors are present in sufficient quantities.

\subsubsection{Cumulative culture masking genes}

Our living conditions have not always been the way they are today. Just two hundred years ago, 89\% of humanity lived in extreme poverty (Ravallion 2016), 88\% were illiterate (van Zanden et al. 2014), and $43 \%$ of children died before they were five years old (Gapminder 2020). Conditions have rapidly improved: rates of extreme poverty are now $10 \%$, illiteracy is down to $14 \%$, and deaths before five years of age are now 4\% (World Bank Group - International Development, Poverty, \& Sustainability 2020; UNESCO Institute for Statistics 2013). Of course, even if most are now better off, our world still suffers from immense global inequality. Given this restriction in historical range combined with the WEIRD sampling problem, it is not clear how well the genetic effects that have been catalogued thus far can be generalized beyond the particular cultural and temporal contexts in which they were studied. And even if this generalizability issue is acknowledged in principle, the bounds on generalizability are unknown.

Genes can be functionally masked by cumulative cultural evolution, and we expect that this masking is extensive and systematic. Cultural masking may help explain the limited portability of polygenic scores across populations (Martin et al. 2019; 2017; Kim et al. 2018). There is nothing too mysterious about this phenomenon: it's what happens when there are multiple evolutionary systems operating within a shared space of biological function. To build upon an example discussed by Deacon (2003): vitamin C is an essential nutrient and its acquisition is thereby an essential biological function. Endogenous synthesis of vitamin $\mathrm{C}$ requires a gene called GLO, and GLO is present across most of the animal kingdom. But because vitamin $\mathrm{C}$ synthesis is metabolically costly, the gene is inactive in some species that have access to sufficient quantities of the nutrient in their diets (Drouin, Godin, and Page 2011). These include taxa such as teleost fishes, guinea pigs, many bats, some passerine birds and anthropoid primates, i.e. monkeys and apes (Chatterjee 1973). Anthropoids for instance occupy a frugivorous niche, and fruits often contain sufficient vitamin C. Here gene function is offloaded onto environmental resources. In turn, this offloading has 
behavioral implications. If a species becomes dependent on its environment ("auxotrophic") for vitamin $\mathrm{C}$, both its behavioral range and evolutionary trajectory become constrained by the availability of the nutrient. Humans are a nice example of this. As our species migrated across the planet, we found ourselves in environments where vitamin $\mathrm{C}$ was in short supply. A deficiency of vitamin $\mathrm{C}$ causes scurvy — the bane of seafarers until the trial-and-error discovery that certain food items like sauerkraut and citrus could prevent ships from being packed with tired, bleeding, toothless, and eventually dead sailors (Lamb, May, and Harrison 2017).

Other species have other ways of obtaining vitamin $\mathrm{C}$ from their environments, and once these alternative pathways are established, the function of $G L O$ becomes masked. A functionally masked gene is removed from the selection pressures that brought it about in the first place, and can therefore be culled from the genome, as evolution tends to do to unused elements (Albalat and Cañestro 2016; Wolf and Koonin 2013). Masking does not necessarily need to be in the direction from culture to genes: we can think of genetic assimilation (Crispo 2007; Waddington 1953) as the same process working in the opposite direction, where a trait that is regularly acquired through learning gradually transfers its locus to the genome (see Baldwin effect; Morgan, Suchow, and Griffiths 2020). Genetic assimilation can eliminate the cost of learning, but only by sacrificing flexibility. The masking of genes by cumulative culture can eliminate the metabolic cost of endogenous synthesis in the case of vitamin $\mathrm{C}$, and perhaps other costs in other cases, but only by sacrificing reliability of outcome.

Culture and genes are tightly intertwined in this manner, but this kind of functional interaction occurs across various levels of biological organization: between genes within the same genomeintragenomic (Phillips 2008), between nuclear and organellar (mitochondria and plastid) genomes-cytonuclear (Sloan et al. 2018), and between host and microbial symbiont genomesbolobiontic (Bordenstein and Theis 2015). Mitochondria, for example, are believed to have undergone extensive reductive evolution, transferring nearly all of their genes to the nuclear genome (Wolf and Koonin 2013; Sloan et al. 2018). Indeed, the residual mitochondrial and nuclear genomes collaboratively assemble "chimeric" proteins (Osada and Akashi 2012). This kind 
of coevolution is not uncommon in the history of life (Laland et al. 2015; Jablonka and Lamb 2005).

Similar to these cross-level interactions, culture and genes are interwoven in the construction of many behavioral traits, making separation effectively impossible. Because these two systems interact within a shared space of phenotypic variation, a focus on only one or the other leaves significant ambiguity in the causal underpinnings of these phenotypes. For example, is language primarily the result of culture or genes? Such a question can be answered only by recourse to both sides of our dual inheritance (Dediu 2011; Christiansen and Chater 2008; Chater, Reali, and Christiansen 2009; Wong et al. 2020; Dediu and Ladd 2007; Deacon 1997). Epistemological biases in the interpretation of the causal loci of phenotypic traits can misinform policy decisions and impede progress in the research and development of beneficial interventions. Given the inherent ambiguity that arises because of gene-culture interaction, it is important to adopt a sufficiently integrative framework to interpret findings.

\subsection{Cultural Simpson's Paradox}

The WEIRD sampling problem, the hidden cluster problem, and the causal locus problem are fairly general issues. But when we consider them jointly, the three problems contribute to a more specific problem that is perhaps not so obvious without a cultural evolutionary perspective. In particular: cultural adaptations that mask genetic effects (causal locus problem) can confound the measurement of genetic effects when the researcher lacks information about the fine-grained distribution of cultural adaptations (hidden cluster problem) and simultaneously lacks access to a sufficiently broad range of samples that could otherwise cut through the cultural confound (WEIRD sampling problem). This set of problems can create a Simpson's paradox (Simpson 1951; Kievit et al. 2013): the association between two variables qualitatively changing after controlling for subgroup structuring.

As an example, consider UV once again. In section 2.1, we discussed how mismatch between genes (skin pigmentation) and ecology (UV levels) can be masked by the cultural diffusion of sunscreen, 
especially in regions with more exposure to sunlight. In other parts of the world, the more important health issue is the set of problems associated with under-exposure to the sun, which causes vitamin $\mathrm{D}$ deficiency. Low vitamin $\mathrm{D}$ is associated with a broad range of risk factors, most significantly for bone integrity but also for muscle strength, autoimmune disease, cardiovascular disease, cancer (Holick 2007), and perhaps COVID-19 (Meltzer et al. 2020; Rhodes et al. 2020). Controlling for skin pigmentation, vitamin D deficiency is correlated with latitude: one US study compared a sample in Erie, Pennsylvania $\left(42^{\circ} \mathrm{N}\right)$ to one in Bradenton, Florida $\left(27^{\circ} \mathrm{N}\right)$ that was matched along many key variables, and found that the northern group had lower levels of serum vitamin $\mathrm{D}$ and were at much greater risk of vitamin $\mathrm{D}$ deficiency than the southern group (Leary et al. 2017). A study in France similarly found that people in more northern regions had lower levels of vitamin $\mathrm{D}$ and a much higher prevalence of vitamin $\mathrm{D}$ deficiency compared to people in more southern regions (Chapuy et al. 1997).

Despite this intuitive pattern within countries, several studies have shown that when we compare across countries within Europe, we see the opposite pattern where people in northern countries have higher levels of serum vitamin D than people in southern countries (Van der Wielen et al. 1995; Lips et al. 2001; Lips 2001). This is the case even when data collection is conducted during the winter months when sunlight is scarce, and even when the data are processed by a central laboratory facility, avoiding confounding by variation in laboratory procedures. What's going on? This inverted pattern may be partly due to genetic factors, e.g., people in southern European countries having more pigmented skin, but a substantial part of it is likely due to culture. As an explanation, various researchers have pointed to the high consumption of fatty fish and cod liver oil in Northern Europe, as well as greater sun-seeking behavior in these countries compared to Mediterranean Europe; policies for vitamin D fortification of foods may also give the northern countries an advantage (Brustad et al. 2004; Mithal et al. 2009; Lips 2007; Pilz et al. 2018). These are potent cultural adaptations: Brustad et al. (2004) fed participants the traditional northern Norwegian fish dish molje three times over a span of two days, and discovered that it had supplied 54 times the recommended daily dosage of vitamin D. Therefore, the relationship between latitude and Vitamin D levels goes one way within a country, and the other way between the countries of Europe. 
Another example of a cultural Simpson's paradox from Europe is excess mortality in winter months compared to non-winter months across countries: excess winter mortality is highest in warmer countries like Portugal and Malta, and lowest in colder countries like Finland and Iceland (Healy 2003; Fowler et al. 2015; McKee 1989). Portugal has the highest excess winter mortality among the countries estimated by Healy (2003) at 28\% while Finland has the lowest at 10\%, despite a much sharper temperature differential between summer and winter months (as well as lower absolute temperature) in Helsinki and Tampere compared to Lisbon and Porto. This has been called the "paradox of excess winter mortality" (Healy 2003). The likely cause is that houses in warmer climate regions tend to be poorly insulated, which causes lower indoor temperatures. Populations in these regions also do not wear appropriate clothing when outdoors in cold weather (Healy 2003; The Eurowinter Group 1997). In south Finland 72\% of people wore hats whilst outdoors at $7^{\circ} \mathrm{C}$, but only $13 \%$ of people in Athens did (The Eurowinter Group 1997). A number of studies show that within countries, regions with colder winters experience higher excess winter mortality (Davie et al. 2007; Aylin et al. 2001): here too there appears to be an inversion when comparing the effect between countries and within countries.

If we had been Martian anthropologists who did not know that the populated landmass known as "Europe" can in fact be broken down into sub-units called "countries", these examples would be standard examples of a Simpson's paradox (Simpson 1951; Kievit et al. 2013). In these cases, the paradox occurs when we do not know how to partition the higher-order population (Europe) into lower-order units. Fortunately, we do know how to partition continents into countries, but in other cases, the relevant units may not always be as easily identifiable as countries or administrative regions.

Countries are territorially exclusive political entities, but we can also see them as cultural groupsclusters of cultural traits that can identified using tools such as $C F_{S T}$. People within a cultural group share more cultural traits than do people between separate cultural groups. All else being equal, there is more mutual cultural influence among individuals within a country than there is among people between countries. Even when we take a powerful social organizing force like religion into 
account, co-residents of a country who belong to different religions are more psychologically similar to each other than to co-religionists who live in different countries, although religion nonetheless also explains a sizable amount of similarity (White, Muthukrishna, and Norenzayan 2020). This suggests that ideas and practices spread more easily within countries than between countries, and that countries constitute cultural clusters that can be used to partition higher-order levels of organization, such as continents. Countries too may in turn be decomposed into relevant cultural clusters-more acutely in places like Africa, where colonial borders were often drawn arbitrarily without respect for ethnolinguistic groups (Michalopoulos and Papaioannou 2020), but clustering is prevalent across many countries as revealed by subnational $C F_{S T}$ analyses (Muthukrishna et al. 2020). To avoid a cultural Simpson's paradox, we must be able to measure cultural clustering.

Hidden clustering creates inferential problems for behavioral genetics precisely because cultural clusters are the most salient unit of organization upon which cultural group dynamics and cultural evolution act (Handley and Mathew 2020). Cultural evolution is fast and potent but bound within cultural clusters to varying degrees. Because different clusters are each independently able to incorporate cultural adaptations, genetic effects can get differentially masked in a cluster-wise fashion. The extent of this masking varies across clusters, and those that are exposed to greater ecological challenges (e.g., higher latitudes) may build up stronger cultural adaptations and undergo deeper masking. When these cultural adaptations not only compensate for the ecological challenge but overcompensate, and do so proportionally to the magnitude of the ecological challenge, the outcome is an inversion of the natural relationship between ecologies and phenotypes - as we saw above in the inverted correlation between latitude and vitamin D (Brustad et al. 2004; Mithal et al. 2009; Lips 2007; Pilz et al. 2018) and between latitude and winter mortality (Healy 2003; Fowler et al. 2015). The cultural Simpson's paradox thus arises when cluster-wise cultural adaptation across an ecological gradient changes the slope of the ecological effect: attenuating, neutralizing, or inverting its directionality depending on the strength of the cultural adaptation relative to the ecological challenge. 
Therefore, the relationship between genes, ecology, and phenotype will often be qualitatively different for humans than it will be for other animals. We should be careful of inferential errors that may derive from neglecting the cluster-wise cultural evolution of environments. Even if the gradient of cultural adaptation does not go as far as inverting the ecology-phenotype relationship and instead takes a more moderate form, it can nonetheless confound measurement of genetic or ecological effects. If the arrangement of the cultural clusters within a population is unknown, such gradients may be difficult to detect in the first place.

To summarize the problems that we have discussed thus far: the WEIRD sampling problem and the hidden cluster problem each obscure the variance structure of a sample-the former by concealing the range of total variation that exists outside of a restricted (possibly unrepresentative) segment, the latter by concealing the heterogeneity within that segment. The causal locus problem allows for differential masking among the heterogeneous sub-groups, and poses a challenge to interpretation of gene function, for example in the form of a cultural Simpson's paradox. Collectively, these problems have long obscured the effect of culture within behavioral genetics. Cultural evolution can help us understand phenotypic distributions in human societies as well as more accurately represent the structure of our nature and nurture.

\section{BEHAVIORAL GENETIC PUZZLES IN LIGHT OF CULTURAL EVOLUTION}

A dual inheritance and cultural evolutionary theoretical framework can help make sense of various puzzles in behavioral genetics. Here we discuss three: differences in heritability across socioeconomic levels, differences in heritability across development, and the Flynn effect.

\subsection{Heritability across socioeconomic levels}

The heritability of IQ is higher among affluent, high socioeconomic status (SES) households than among poorer, low-SES households in some societies (sometimes referred to as the Scarr-Rowe effect; Scarr-Salapatek 1971; Rowe, Jacobson, and Van den Oord 1999), but the relationship between SES and heritability is mixed in other societies (Nisbett et al. 2012; Hanscombe et al. 
2012; van der Sluis et al. 2008; Turkheimer et al. 2003; Giangrande et al. 2019; Platt et al. 2019). A cultural evolutionary perspective can shed light on these findings.

\subsubsection{Discrepancies in cultural transmission across societies}

A meta-analysis (Tucker-Drob and Bates 2016) found the gene $\times$ SES interaction on IQ in a subset of US samples, but not in samples from Europe and Australia. Pooling the US studies, the authors found an effect size that corresponds to a heritability estimate of 0.61 at 2 standard deviations above the mean SES but only 0.26 at 2 standard deviations below the mean. In western Europe and Australia, heritability is more uniform. The cause of this interaction is still debated.

Several researchers (e.g., Bates, Lewis, and Weiss 2013; Beam et al. 2015; Tucker-Drob, Briley, and Harden 2013) have suggested that gene-environment correlation via phenotype-toenvironment transmission, otherwise referred to as 'reciprocal causation', is the most likely explanation. By this explanation, those with genes well suited to a task can better nurture their skills in a wealthier environment than in a poorer environment. That is, initially small differences in genetic potential become gradually amplified over time due to the iterative matching of environments to abilities: an increase in expressed ability brings forth new environmental conditions that enable further growth along that dimension (Dickens and Flynn 2001; Bronfenbrenner and Ceci 1994; Scarr 1992). Such processes can increase genetic heritability, but through reciprocal shaping between genetic potential and environment, rather than through innately specified ability levels. The reasoning is that high-SES households are able to provide environments that do this more effectively and are thereby able to let genetic potential be more reliably associated with corresponding outcomes, lifting heritability as a result. While such reciprocal causation may indeed be occurring, reconciling this explanation with the findings from Europe and Australia seems more challenging or at least incomplete.

Heritability is a function of the variability in culture, which is shaped by cultural group dynamics and cultural evolutionary forces. In the United States, the differences between, for example, school and home environments among high-SES households is likely to be small relative to differences 
between school and home environments among low-SES households, where factors such as school lotteries can dramatically affect the cultural input. In contrast, the cultural environment is less unequal in western Europe and Australia, where, for example, high quality schools are available across SES. Where these two explanations make different predictions is for poorer countries. The reciprocal causation explanation would predict low heritability in poorer countries. The cultural evolution of genetic heritability explanation would instead predict high heritability where there is equal access to similarly poor schools and household conditions, but low heritability if inequality is high.

Although environmental variability is commonly construed through the framework of economic inequality, we can also look at it through the lens of cultural variability. For example, even if a society maintains economic equality, it may still contain cultural sub-divisions that lead to multiple clusters each with different traditions and behaviors. When this cultural clustering creates differences in the level of some given trait between clusters, the heritability of this trait decreases in accordance with the strength of the clustering.

We predict that networks of cultural transmission are more fractured (i.e., contain a higher density of cultural clusters) in low-SES households than they are in high-SES households comparing within a country, at least for (but not limited to) transmission of cultural information and influence that impacts traits in domains like cognitive ability. We also predict that these cultural networks are more fractured in the US than in Australia and Europe, with the greatest fracturing expected in low-SES US communities. Moreover, we predict that these differences across groups will explain at least some portion of the interaction between SES and heritability in the measurement of IQ. These effects could be tested through causal identification techniques, including randomized controlled trials or natural experiments such as school lotteries.

A number of studies have examined unstandardized variance components to make sense of the forces that are driving the gene $\times$ SES interaction effects for cognitive ability, but the results have been mixed. In some studies, the interaction is apparently due to an increase in variance explained by the shared environment (the $\mathrm{C}$ component of the ACE model) in lower-SES households 
compared to higher-SES households (e.g., Hanscombe et al. 2012; Kremen et al. 2005), while other studies suggest that it is instead due to a decrease in variance explained additively by genes (the A component of the ACE model) (e.g., Bates, Lewis, and Weiss 2013; Kirkpatrick, McGue, and Iacono 2015). The shared environment explanation is more consistent with cultural dynamics, although reality may be more complicated with other processes such as reciprocal causation (Dickens and Flynn 2001; Bronfenbrenner and Ceci 1994; Scarr 1992) contributing to the same effect through other pathways, such as via moderation of the additive genetic component. Moreover, we would predict that this environmental variance would be reduced if cultural opportunities and transmission networks among low-SES households became more broadly connected, supporting greater cultural homogeneity. This would be true, even if the level of poverty or other indicators of well-being remained just as low. That is, this effect is not about poverty or deprivation per se-heritability can be high even among lower-SES groups-but more about cultural clustering. Simply moving neighborhoods can vastly improve life outcomes for low-SES Americans (Chetty and Hendren 2018b; 2018a), but we would not predict such large effects for high-SES Americans.

In general, we predict that Scarr-Rowe-like discrepancies in the heritability of IQ will be found when comparing groupings that vary in their degree of within-group cultural clustering (or homogeneity) whenever this clustering imposes barriers upon the cultural transmission of cognitive ability. The more clustered society will be associated with a lower heritability for the trait; this effect would be expected across many culturally transmissible traits beyond IQ as well. When disaggregated by cultural cluster (e.g., SES), we should expect lower heritability among the more culturally diverse and/or more culturally clustered subgroup.

\subsubsection{Discrepancies in environmental variation between humans and other animals}

Comparing these SES effects in humans with similar experiments in rodents offers additional insight. Sauce et al. (2018) found that mice reared in an enriched environment exhibited lower heritability of a rodent analog of general intelligence (for standard rodent learning tasks such as odor discrimination and navigation) than did mice reared in a control environment, with a 
heritability of 0.15 in the enriched group versus 0.55 in the controls. The directionality of this difference is opposite of what we have described above for the human literature, and the opposite of what the reciprocal causation explanation would suggest (Dickens and Flynn 2001; Bronfenbrenner and Ceci 1994; Scarr 1992). If we assume that this type of environmental enrichment paradigm can be mapped onto differences between high- and low-SES environments in humans, as has been argued (Rosenzweig and Bennett 1996; Hackman, Farah, and Meaney 2010), the reported discrepancy between rodent and human studies might appear surprising.

Non-genetic phenotypic variation in mice appears to be generated in part by initially small, random differences in experience being amplified over the course of development (Kempermann 2019) complex environments enable greater amplification of these initial differences and hence 'individualization', whether the environmental complexity is of a physical (Freund et al. 2013) or social nature (Shemesh et al. 2013). In these studies, both genes (due to inbreeding) and the shared environment are controlled. It is therefore the non-shared environment, or individually unique forms of experience, that is driving this differentiation. The enriched environment enables a greater range of variation in experience and as a consequence, genetic effects are proportionally reduced.

Compare this to humans, where cultural transmission gradually homogenizes the environment within a cultural cluster (though not necessarily between cultural clusters), and to varying degrees across clusters as well. We predict that high-SES US households as well as households within Australia and European countries are less culturally fragmented than low-SES US households, leading to higher environmental similarity within Australians, Europeans, and high-SES Americans respectively (but not necessarily high similarity between the groups). In other words, relatively enriched environments (high-SES or Australia/Europe) are also those that are more culturally homogenous. This in turn may explain why enriched environments would be negatively associated with heritability in mice (Sauce et al. 2018) but positively associated with heritability in humans at least under certain conditions (Tucker-Drob and Bates 2016). Our prediction is that the opposite effect between the two species arises because in humans, the amplification of initial differences in experience by complex environments (individualization) is overwhelmed by the homogenizing effect of cultural learning and group dynamics (for example, through oblique 
transmission from favored models in the previous generation and horizontal transmission from peers). The more variation is generated by enriched environments and individualization, the more incentive there is to learn from successful individuals or from those who are likely to have learned from successful individuals themselves. Cultural diffusion systematically squashes the variation created by innovation (see Appendix for a model of such dynamics).

A cultural evolutionary approach to heritability distinguishes between humans and other animals in ways that a standard behavioral genetics approach does not. Whereas behavioral genetics provides no clear reason to differentiate analysis of genetic effects in humans from genetic effects in non-human animals, cultural evolutionary theory explicitly formalizes the difference between humans and other animals: our extensive dual inheritance (Boyd and Richerson 1985). The human-specific predictions that arise from a cultural evolutionary behavioral genetics offer clear tests for our hypotheses. The approach may thus also be validated by comparative behavioral genetic analyses between humans and non-human animals. This research direction may help make sense of some of the limitations of non-human animal models, in cases where cultural adaptations create unexpected gaps in generalizability despite sufficient phylogenetic conservation of relevant phenotypes.

\subsection{Heritability across development}

Cultural heterogeneity may also vary across development. Because culture typically acts as a phenotypic homogenizer within cultural clusters, we should be able to detect the influence of culture across development in the form of changes in genetic heritability. How we learn and who we learn from changes over the lifespan. One especially important transition is the shift from learning primarily from parents and other family members to learning from more distant models who are selected from a broader swath of society (Cavalli-Sforza and Feldman 1981). In the first of these two phases, there is less choice in what to learn, and much of the acquired knowledge is passed down through the same route as genetic information-from parent to child-by vertical transmission. In the second phase, the child is more independent, and has the opportunity to update what they have learned from a broader range of models, using learning strategies to decide whom 
to learn from, by oblique transmission. This expansion in learning models is essential for cumulative cultural evolution (Enquist et al. 2010), and may be facilitated by adaptations such as early weaning (Finlay and Uchiyama 2020; Hawkes and Finlay 2018). Building on Cavalli-Sforza and Feldman (19273), who show that vertical transmission inflates heritability estimates by increasing similarity between caretakers and children, our argument focuses on how broad, community-level oblique and horizontal transmission can either inflate or deflate heritability by unmasking or masking genetic effects. Both processes are likely to be operating simultaneously, although if oblique transmission is broad enough, it may weaken the confounding effect of vertical transmission.

This transition from vertical to oblique learning moves the child from the idiosyncrasies of their parents and household to the larger environment they now have in common with other adolescents and young adults. When the child is primarily relying on vertical transmission, the characteristics of their household plays a larger role in explaining variation in cultural input, in which case we should expect a high proportion of phenotypic variance to be explained by the shared (home) environment in twin studies. When the child switches to oblique learning, they now share more common influences with other children. Insofar as these traits unmask genetic differences, this would be expected to reduce environmental variation across the population and increase heritability.

This reasoning implies that for phenotypic traits that are molded in real-time by the current shared environment instead of by the persisting effects of earlier parental influence, heritability should increase at this later life stage. Indeed, this is precisely what Hatemi et al. (2009) find in the case of political orientation, or where one lies on a progressive-conservative spectrum, measured in a US sample by questionnaire (Figure 3). Monozygotic (MZ) and dizygotic (DZ) twin pairs are both equally similar from middle childhood up to early adulthood, although the degree of twin similarity increases over time for both. Right around the age at which American children leave home, this pattern is broken, and the phenotypic correlations drop precipitously in DZ twins while remaining steady in $\mathrm{MZ}$ twins, and this discrepancy persists for the rest of the lifespan. The drop in $\mathrm{DZ}$ but not $\mathrm{MZ}$ correlation at this age suggests that the shared home environment exerts a convergent influence for both twin types early in life, but that once this influence is removed, genetic effects become unmasked and able to guide political attitudes independently from the 
shared environment. More phenotypic variance is explained by genes from this point onward, thus boosting heritability. In countries like Italy and Croatia, where the mean age of leaving the parental household is past 30 (European Statistical Office 2020), we would predict the developmental time course of heritability to reflect this later independence relative to American samples. Note that the present example has the same overall structure as the literacy example discussed earlier (Samuelsson et al. 2008), with heritability increasing as cultural influences from outside of the home environment kick in. Both examples indicate that heritability can be an index of shared life history and communal structure.

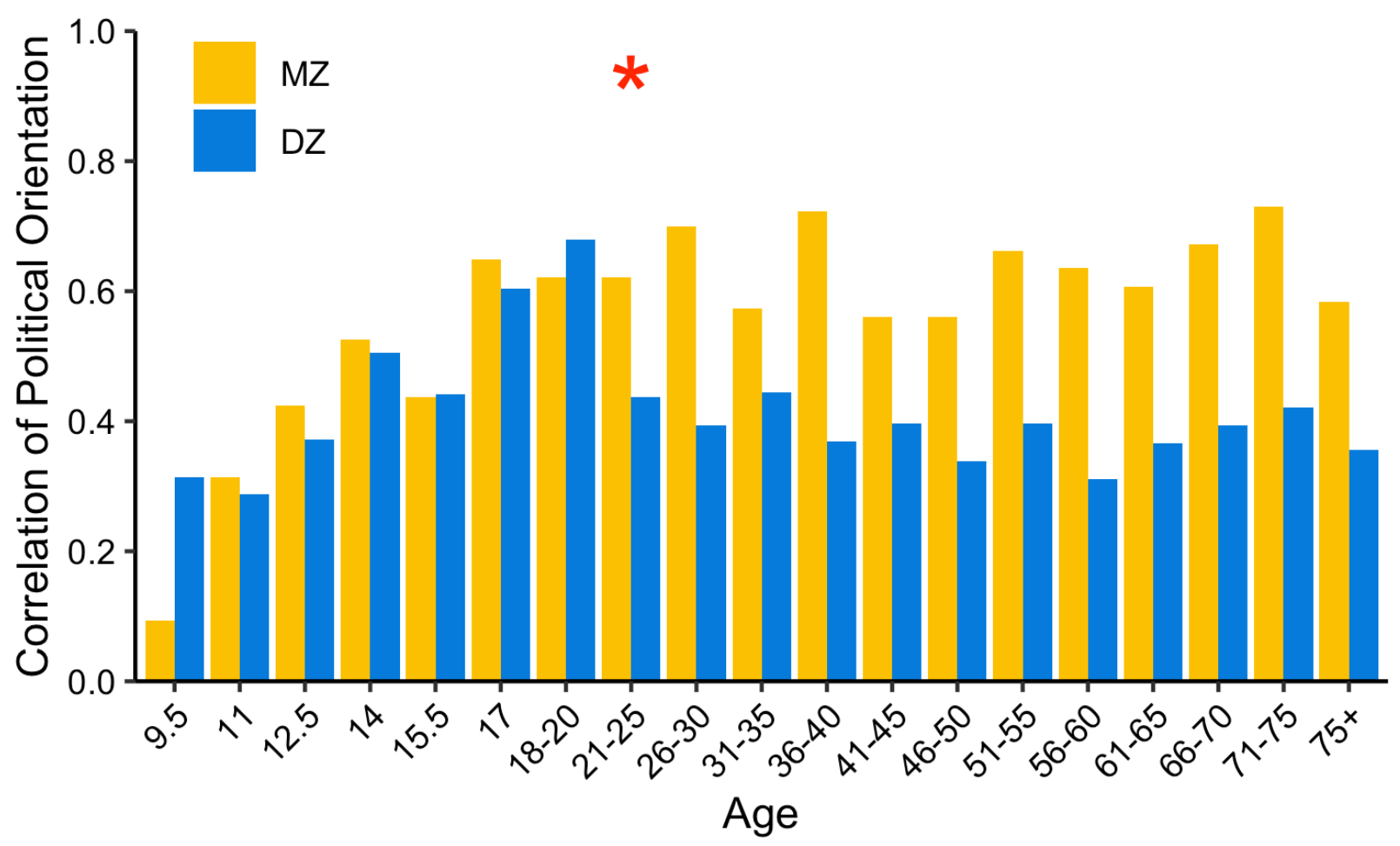

Figure 3: Twin concordances in political orientation. In middle to late childhood, within-twin correlations for reported political orientation are roughly the same between Monozygotic (MZ) and dizygotic (DZ) twin pairs in a US sample. In the early 20s, shortly after many US youth leave home for the first time to attend university, the correlation drops for the DZ twins but not for the $\mathrm{MZ}$ twins (identified with red asterisk). This shift corresponds to a sudden rise in heritability, as genetic similarity now predicts similarity in political orientation. When the effect of the home environment is weakened and replaced with more diverse cultural input, the effect of genes becomes unmasked and separates the phenotypic concordances between the two twin types. Horizontal axis indicates age, vertical axis indicates percentage twin concordances in political orientation. Figure reproduced from Hatemi et al. (2009).

Although the use of shared household environment to analyze twin data is a standard methodological convention, the household is in fact just one among many groupings of cultural 
organization that generate environmental convergence (Harris 1995). Households may be the most potent cultural grouping for some phenotypic traits, but other groupings may have significant impact as well for specific kinds of traits. These may include schools, peer-groups, sports teams, religious communities; society-wide groupings such as different mass media and popular culture; more diffuse groupings that are organized around particular sets of values such as political ideology or professional values; and possibly new kinds of groupings such as online communities.

Separating out the effect of household from the effect of genes is typically considered to be an explanatory goal, but there may be further phenotypic variance that could be meaningfully explained if we were able to match phenotypes to other relevant cultural groupings and therefore to other sources of cultural influence. Twins share 'common environments' across multiple scales of social organization in this manner, but when phenotypic similarity is engendered by cultural groupings that extend beyond the household, the resulting correlations will usually be relegated to the broad category of 'nonshared environment', unless membership for example happens to align with household structure (Plomin and Daniels 1987; Plomin, Asbury, and Dunn 2001). Although the nonshared environment is typically discussed as environmental exposure that is specific to the individual, it remains possible that there are multiple layers of communal structure embedded within this variance component.

This point becomes somewhat obvious when we consider systems outside of the European Marriage Pattern (Henrich 2020), such as the Israeli kibbutzim (Lieblich 2010), the "walking marriages" of the Mosuo of southwest China (Ji et al. 2013), or the communal child rearing of the Ache of eastern Paraguay or of the Hiwi of Venezuela (Hill and Hurtado 2009). In such societies, similarity of developmental environments may not be adequately parsed at the unit of the household (Kăĝitçibaşi 1984). The Western notion that socialization takes place primarily in the household may not apply even to Western societies (Harris 1995). If this is the case, then the strategy of looking for phenotypic correlations within the household that are unexplained by genes may be appropriate for answering questions about the specific influence of the household, but is insufficient for answering questions about nature-nurture more broadly. 
One potential way to overcome this limitation is to map out clusters of cultural similarity within a society using a tool like Cultural $F_{S T}$ (Muthukrishna et al. 2020), explained above, and match this finer-grained population structure with genetic data. If assignment to a cluster explains phenotypic similarity over and above that of the shared household, for instance, such methods could be leveraged to capture a broader picture of the environmental factors that guide phenotypic development. If we were able to track the changing organization of these cultural clusters over time, it would allow us to follow the dynamic trajectory of environmental structure and partition this out from measurements of genetic effects longitudinally. We expect social phenomena like urbanization and demographic change to be associated with change in these cultural clusters over time.

General intelligence is another trait whose heritability is known to change over the course of development (Haworth et al. 2010; Briley and Tucker-Drob 2013; for a number of other traits see Bergen, Gardner, and Kendler 2007). This takes the form of a steady increase from childhood through adolescence all the way to early adulthood, after which it remains more or less steady over the lifespan. Although estimates vary, one meta-analysis (Haworth et al. 2010) put the heritability of general intelligence at 0.41 in childhood and 0.66 in adulthood. Explanations for this pattern typically invoke a combination of (1) gradual activation of relevant genes over the course of brain development and (2) active gene-environment correlation or 'reciprocal causation' (Bouchard 2013; Haworth et al. 2010; Plomin et al. 2016; Tucker-Drob, Briley, and Harden 2013).

In contrast, a cultural evolutionary perspective would attribute the rise in the heritability of IQ to the developmental time course of cultural influence. One clear testable prediction is that in a society with different constraints on the development of cultural learning, as in the above example of political orientation, the developmental trajectory of heritability would also differ. A society that engages with communal childrearing immediately from infancy will impose a different set of developmental constraints (and opportunities) compared to a typical Western society. Even in Western societies, cultural development may look very different from now just a few decades into the future, if information technology continues to transform children's networks of social interaction and influence. A cultural evolutionary explanation can help make sense of the relevant 
data. For example, we predict that sharp changes in heritability will map onto sharp changes in an individual's cultural environment (e.g. the start of school, university, or military service). These milestones may shift due to policy changes, allowing for causal tests of this hypothesis. If, for example, children start higher education later, then large increases in heritability should also occur later.

Whether or not one agrees with our construal of these particular examples, it is logical that genetic effects for many traits become confounded with communal structure unless the developmental time course of cultural influence is properly accounted for. Cultural evolutionary theory predicts that over the life course, cultural influence begins from a primarily parental setting and progressively expands its scope into the greater community, diversifying the models from which an individual can learn (Fogarty, Creanza, and Feldman 2019; Muthukrishna et al. 2018). Insofar as this occurs, we predict a general increase in heritability over the lifespan for culturally malleable traits. The slope of this increase would depend upon cultural parameters and vary by society. Conversely, if there is a correlation between communal structure and the heritability of some trait, this may serve as an index of the cultural malleability of that trait. Our goal here is to bring attention to the conceptual and analytic power that cultural evolution can contribute to developmental behavioral genetics.

\subsection{The Flynn effect}

The Flynn effect describes the rise in IQ test scores over time (Flynn 1984; 1987)—roughly 2 to 3 IQ points per decade on average around the world (Trahan et al. 2014; Pietschnig and Voracek 2015; Flynn 2009). The rate of increase differs between countries, being largest in countries that have recently started modernizing, and smallest in countries that had attained modernization by the beginning of the 20th century (for review, see Nisbett et al. 2012). In some countries in Northern and Western Europe including Denmark, the Netherlands, and the United Kingdom, there is evidence that the Flynn effect has been slowing down and even reversing in recent decades (Dutton, van der Linden, and Lynn 2016). This negative Flynn effect is even less well understood than the positive Flynn effect. Bratsberg and Rogeberg (2018) find that in Norway, the negative 
Flynn Effect is found within families (between siblings), thereby making it unlikely to be explained by demographic changes or immigration, and instead supporting an environmental explanation.

There is no consensus regarding the cause of the Flynn effect, but given the recent and rapid increase, genetic explanations are unlikely. Various hypotheses include increases in test familiarity, improvements in education, sophistication of the technological and media environment, better nutrition, decreasing family size, and slowing life history, and increased out-breeding or 'hybrid vigor' (Bratsberg and Rogeberg 2018; Clark, Lawlor-Savage, and Goghari 2016; Nisbett et al. 2012; Pietschnig and Voracek 2015; Trahan et al. 2014; Johnson 2006; Joshi et al. 2015; Woodley 2012).

Flynn (2007) and Greenfield (1998; 2009) suggest that the effect is caused by a rapid worldwide increase of cultural practices, technologies, and environments that promote abstract cognitive processing as opposed to more traditional forms of concrete, pragmatic thinking. Some examples explored by these authors included urbanization, mass media, video games, education style, counterfactual thinking, and white-collar occupations. This account is mostly consistent with a cultural evolutionary explanation, which would suggest that intelligence is not just about hardware-genes, parasites, pathogens, pollution, and nutrition affecting health and brain development, but also software - the increasingly complex cultural package we acquire from our societies (Mesoudi 2011; Gordon 2018; Bloom 2020; Jones 2020). By this account, not only is the idea of a culture-free IQ test implausible, but so too is the idea of culture-free IQ(for discussion, see Muthukrishna and Henrich 2016). Indeed, the largest Flynn effect can be seen on the supposedly culture-free Raven's matrices (Nisbett et al. 2012; Flynn 2007), and on tests for fluid IQ rather than crystallized IQ (Pietschnig and Voracek 2015). When it comes to heritability, subtests of IQ that are more culturally influenced are more heritable (Kan et al. 2013).

Beyond the diffusion of specific traits and abilities, a cultural evolutionary explanation also highlights how the Flynn effect is driven by the reorganization of cultural transmission pathways themselves. The introduction and improvement of formal schooling is one major instance of reorganization in cultural transmission that is also known to positively impact IQ (Ceci 1991; 
Brinch and Galloway 2012; Davis 2014; Ritchie and Tucker-Drob 2018). Greenfield (1998) describes how IQ scores in some rural US towns in the early 20th century increased rapidly at the same time as a number of coordinated changes in infrastructure, including better access to urban areas and new, high-quality road systems. Such enhancements in social connectivity directly translate into cultural connectivity, allowing for the influx and diffusion of psychological and behavioral traits that are considered valuable within the broader society. In much of the modern world, the kind of abstract information-processing ability measured by IQ tests is considered valuable, as it is useful in various white-collar professions that are typical of WEIRD societies. The Flynn effect therefore captures the progressive enhancements in cultural connectivity that have been occurring around the world due to improvements in various domains of infrastructure and technology including transportation, urbanization, education, and media. Global IQ rises in response to both the invention of relevant cultural traits and the enrichment of cultural transmission networks that carry those traits.

One clear test of this cumulative culture explanation for the Flynn effect would be to randomly assign children to attend or avoid formal schooling. Such a test would be highly unethical and differences in school attendance and quality are typically associated with various kinds of deprivation. Where policy changes have changed years of schooling in ways that lend themselves to causal identification through a natural experiment, an increase in formal education has been shown to cause an increase in IQ (Brinch and Galloway 2012; Ritchie and Tucker-Drob 2018). A recent test by Davis et al. (2020) offers even clearer data. Children, but not adult IQperformance is compared within age groups, because we assume children perform better as they get older. Exploiting a natural experiment where access to education was uncorrelated with SES, nutritional status and acculturation, Davis and colleagues show that without access to education, there is no correlation between age and IQ test performance. With moderate access, we see a moderate slope and with more access, a slope similar to the West.

\section{CULTURAL EVOLUTIONARY BEHAVIORAL GENETICS}

Behavioral genetics offers a powerful empirical approach to understanding human behavior, but 
since the advent of whole-genome methods, its strategy appears to lean toward the notion that with enough data, ground truths about human nature and nurture, if only for some traits, will be revealed. Data alone is not enough (Muthukrishna and Henrich 2019); the thrust of our theoretical case is that human psychology and behavior have a large cultural component that has been changing over history (Muthukrishna, Henrich, and Slingerland 2021; Nunn 2020; Henrich 2020; 2016; M Chudek, Muthukrishna, and Henrich 2015; Laland 2018; Wilson 2019; Boyd and Richerson 1985; Boyd 2018). Most recently our psychology has been shaped by the advent of writing, numeracy, different types of agriculture, the Industrial Revolution, the Internet, and smart phones (Firth et al. 2019; Wilmer, Sherman, and Chein 2017; Ong 1982; Talhelm et al. 2014; Uskul, Kitayama, and Nisbett 2008; Domahs et al. 2010). As new adaptive traits emerge (Muthukrishna and Henrich 2016), initially those who possess these traits will have an advantage, as in the case of access to new food sources, better healthcare, more efficient technologies, or easier methods of learning. But eventually the traits will reach fixation in the population through the processes of cultural diffusion (Henrich and Broesch 2011; Muthukrishna, Morgan, and Henrich 2016), at least until they are unseated by subsequent innovations (Kolodny, Creanza, and Feldman 2015; Muthukrishna and Henrich 2016). We predict that these cultural dynamics are reflected in heritability estimates.

As any geneticist knows, genetic heritability is a function of the variability in the environment, variability in genes, and variability in the phenotype. There is little to predict if the phenotype is homogenous, as in the number of fingers or kidneys. There is little to predict with if the environment or genes are homogenous. But what is factored into the environment includes not only the physical ecology, but also the cultural environment. While variance in genes and ecology may be relatively stable, the variance in the cultural environment is continually changing through the processes of cultural evolution. Genetic heritability estimates are highly affected by not only cultural diversity and cultural clustering, but also by factors that affect cultural transmission such as sociality (Muthukrishna et al. 2014), transmission fidelity (Morgan et al. 2015), tolerance for variation (Muthukrishna and Henrich 2016), population structure (Henrich 2004a), and social network topology (Derex and Mesoudi 2020; Muthukrishna and Schaller 2020). Under most empirical conditions, behavioral genetics underestimates the contribution of culture, including in 
estimates of heritability. We don't disagree with the findings in these fields or the data used, but instead argue that more nuance is required in how they are interpreted. Our dual inheritance demands that a genetic account of human psychology and behavior must also account for culture and cultural evolution.

\subsection{Toward a dynamic model of environment}

We are surrounded by the products of culture yet are generally unaware of the generative processes that bring such complex objects and conditions into existence. Cultural transmission spans broad networks of interconnected individuals, as well as deep time scales of inheritance. Each individual experiences just a snapshot, leaving the global mechanics opaque. Thus, each of us is left with an intuition that our world is largely the world, which perhaps explains why the extent of the WEIRD people problem went unappreciated for so long, even a decade after publication of Henrich et al. (2010) (Nielsen et al. 2017; Pollet and Saxton 2019; Tiokhin et al. 2019; Barrett 2020; Apicella, Norenzayan, and Henrich 2020). From this limited vantage point, we evaluate questions such as the relative contributions of nature versus nurture. But our understanding of 'nurture' remains fundamentally anchored in our restricted experience of being enculturated into a particular environment, which leads us to implicitly see environmental features shared by members of our community as factors to be held constant, while our variables of interest-be they the absence of a parent, a childhood illness, birth into nobility, or a polygenic score-become matched to outcomes in our predictive models. Such models may be informal or formal, either encountered in community gossip (“children raised by single parents usually become...”) or in scientific journals ("growth mindset interventions predict..."; Sisk et al. 2018 ). Our need for causal explanations (Penn and Povinelli 2007; Gopnik et al. 2004) meets our tendency to essentialize people and groups, where genes offer a better essentialist vehicle than the environment (Heine 2017; DarNimrod and Heine 2011).

\footnotetext{
${ }^{9}$ As an aside, that growth mindset might only replicate among low-SES or at-risk students (if it replicates at all) fits with the general point that prediction is a function of variability. In this case, where there is a deficit, interventions may work, but where there is not, the potential gains are marginal or non-existent.
} 
The importance of limiting behavioral genetic findings to the reference population was famously argued for by Lewontin (1970), and remains a caveat for the analysis of genetic effects. But it is far less appreciated that the reason why a multitude of phenotypic factors can be successfully held constant (or controlled for) in the first place is, in large part, due to the convergent force of cultural learning. Lewontin (1970), in his counterargument to Jensen's (1969) controversial article that argued for the innateness of IQ, used inbred corn and a uniformly acting nutrient solution as his rhetorical props for explaining the environmental sensitivity of genetic effects. Domesticated crops experience a more homogenous environment not by accident, but as a product of human cumulative culture. Lewontin's famous example is thus an unintentional illustration of how culture can generate at times extreme phenotypic convergence in significant features of the environmenteither our own or of our domesticated flora and fauna.

We are all aware of gene-environment interactions (Hunter 2005; Moffitt, Caspi, and Rutter 2005; Lewontin 1970), but we still tend to focus on what is predictive in our statistical models, which are constructed in a particular population, environment, and period of time but whose apparent lessons are commonly generalized beyond these contexts (e.g. the effects of an educational intervention). These models typically do not capture how the relevant environments are distributed within and between populations or how (or why) one type of environment transitions into another-'environment' is simply given as an exogenous variable. The cultural evolutionary approach forces us to explicitly recognize that human environments do not just happen to fall into place; they are rather the outcome of a dynamic, adaptive process that responds to both environmental and genetic factors. The literature on gene-environment interaction already recognizes genes and environments as non-orthogonal, but dependencies between the two are likely to be tighter and more prevalent than would be expected in a culture-free framework. This dynamic view of the environment also suggests that the problem of limited portability of polygenic scores across populations (Martin et al. 2019; 2017; Kim et al. 2018) is also likely to be a problem across historical time in a single population, as the issue is not just about differences in nucleotides across groups but also about the build-up of the cultural environment. 
If we are to accommodate culture, the environment can no longer be treated as a static projection plane over which active elements (i.e., genes and $\mathrm{G} \times \mathrm{E}$ interactions) drop their shadows. Instead, both genes and environment - the latter animated by cultural dynamics-are in motion with respect to each other (as an example, see language-brain coevolution; Christiansen 1994; Christiansen and Chater 2008; Deacon 1997; and cultural niche construction; Laland, OdlingSmee, and Feldman 2001; Laland and O'Brien 2011). An environment can be used as a reference frame against which to judge the effect of genes, but this is done for pragmatic purposes and not because environments are intrinsically fixed (Haworth and Davis 2014). We might take our cue from James Gibson's contribution to the study of vision, which he summarizes in the following manner:

The standard approach to vision begins with the eye fixed and exposed to a momentary pattern of stimuli... The ecological approach to visual perception works from the opposite end. It begins with the flowing array of the observer who walks from one vista to another, moves around an object of interest, and can approach it for scrutiny, thus extracting the invariants that underlie the changing perspective structure (Gibson 1979, p.303).

Gibson recognized that environmental change is not noise, but rather the very medium through which the scientist obtains knowledge about visual function. Our argument presents an analogous approach to the study of genes.

\subsection{Toward a culturally situated understanding of intelligence}

The genetic underpinnings of intelligence have roots going back to the beginning of behavioral genetics (Galton 1869; 1874) and have been fiercely debated since at least Jensen (1969) and Lewontin (1970). The topic remains contentious, but a dual inheritance perspective cuts through some of this debate. Here we summarize some key points.

IQ appears heritable—often measured at around 0.4 in toddlers and increasing up to 0.7 or 0.8 in adults (Bouchard 2009; Bergen, Gardner, and Kendler 2007). But as we and others have discussed, 
a high heritability score does not necessarily tell us whether a trait is primarily genetic; high heritability can also be an indicator of environmental homogeneity. Intelligence is a function of both our hardware (brain) and our software (culture) (Heyes 2018; Hutchins 1995; Vygotsky 1980), and the software has been changing far more and far more rapidly than has the hardware (Uchiyama and Muthukrishna in press). Genes certainly contribute to the size and organization of our brains-indeed, the Cultural Brain Hypothesis predicts a strong selection pressure for larger brains (Muthukrishna et al. 2018), still evident in the rapid increase in emergency birth interventions as a function of head size (Lipschuetz et al. 2015). But those genes are explaining residual phenotypic variation only after accounting for environmental factors that also affect the quality of neural hardware, such as nutrition (Lynn 1990; Stoch et al. 1982), parasites (JardimBotelho et al. 2008), air pollution (Zhang, Chen, and Zhang 2018), and lead exposure (Needleman and Gatsonis 1990; Wasserman et al. 1997). All are known to influence intelligence, but in societies that have been able to minimize variation on such factors through cultural diffusion, the environmental effect is also minimized. And it is not only such physical and physiological variables: changes in and diffusion of the cultural package delivered by schooling (Ceci 1991; Davis 2014; Ritchie and Tucker-Drob 2018; Davis et al. 2020) and our ever more complex entertainment media (Johnson 2006; Greenfield 2009) also reduce the variation to be explained. All these processes unfold outside of any genetic changes to our neural hardware.

Recent, high-powered GWAS have found that genes associated with intelligence are expressed predominantly in the central nervous system (Sniekers et al. 2017; Savage et al. 2018; Davies et al. 2018), but these findings too only explain the residual variation that remains after cumulative culture has reduced variation across many other variables—such as pathogens, parasites, and nutrition - that would otherwise account for huge portions of variation on IQ test performance. The expression of "intelligence genes" may cluster inside the head (Savage et al. 2018; Davies et al. 2018; Sniekers et al. 2017), but this expression profile cannot be meaningfully evaluated without first considering the prior contributions of cumulative culture, which are invisible to standard methods in behavioral genetics. We predict that in general, the set of identified genes that explain human traits such as intelligence is liable to change as a result of cumulative cultural evolution, both across cultures and across time within a single culture. Gene-phenotype mappings for 
culturally modifiable traits in humans are typically more transient than they are in other species, whose gene-phenotype mappings will typically (but not exclusively; see niche construction Laland, Matthews, and Feldman 2016) only change at the slower timescales of genetic evolution or of passive ecological change. Just as limited sampling across historical time has obscured this difference in the temporal horizon of genetic effects across humans and nonhuman animals, the WEIRD sampling problem has obscured differences between humans and nonhumans with respect to within-species generalizability as well.

Even if intelligence is highly heritable in humans, this does not indicate that its genomic substrate works independently of the environment. The cultural environment can amplify heritability, and the degree of this amplification covaries with the extent to which a society has been able to reduce variation in physical, physiological, and informational factors that impact the phenotype. Developed countries, almost by definition, have been most successful in reducing this variation. This flattening of variation is an outcome brought about by expanded networks of cultural influence and greater cultural connectivity, both due in part to technological innovation and in part to culturally evolving social norms and institutions. High heritability of intelligence is therefore most likely to reflect the effect of the cultural environment in these societies. As we argue in our discussion of the Flynn Effect, cultural traits associated with high IQ are themselves transmitted through these cultural networks.

If a society demonstrates high heritability of some trait together with a high relative level of the trait, this combination may give the strong impression of genetic advantage, but the argument that we have developed in this target article points to a different kind of explanation. Comparisons of mean IQ across countries, or 'National IQ' (Lynn and Vanhanen 2012), have been under scrutiny recently, but apart from the various methodological flaws that have been pointed out (e.g., Ebbesen 2020), the non-obvious role of the cultural environment in shaping both the trait and its measurement should be seriously considered in this discussion as well. Epistemic issues like the cultural Simpson's paradox should also be considered in cross-national analysis, as it can create associations between groups and traits that are a product of the cultural environment even when it appears otherwise. The examples that we use to illustrate the paradox - vitamin D deficiency and 
excess winter mortality (Section 3.4) - are easy to grasp, but actual instantiations of the cultural Simpson's paradox are likely to be less straightforward.

The cultural evolutionary framework possesses unique strength in providing these kinds of explanatory strategies, as well as a general theoretical basis for understanding the formation and distribution of psychological traits such as intelligence. The approach that we develop here builds upon earlier, culturally situated approaches to intelligence (Berry 1972; Patricia M Greenfield 1998; Cole 1998; R. E. Nisbett 2009), but goes further in incorporating the population dynamics of cumulative culture, which offers greater clarity in how traits like IQ are shaped by non-genetic processes that span generational time.

\section{CONCLUSION}

Genetics is indeed in a peculiarly favoured condition in that Providence has shielded the geneticist from many of the difficulties of a reliably controlled comparison. The different genotypes possible from the same mating have been beautifully randomised by the meiotic process. A more perfect control of conditions is scarcely possible, than that of different genotypes appearing in the same litter. (Fisher 1952)

Above, Sir Ronald Fisher exalts the inferential purity that is afforded by the powerful pairing of sexual recombination with simultaneous multiple birth, which conveniently flattens environmental variation. But of course, this purity becomes progressively degraded with age, as environmental effects channel offspring through different developmental trajectories. Even among inbred, genetically identical mice who cohabit an experimentally controlled space, self-organizing trajectories of environmental experience result in clear differentiation in phenotypes like exploration, sociality, play behavior, and postnatal neurogenesis (Freund et al. 2013; 2015; Shemesh et al. 2013).

Humans trajectories differentiate so much more. We inhabit almost every ecosystem on Earth, not by speciating as many animals do, but through cultural adaptation, opening different developmental pathways in different societies. But even within a single society, our massive specialization leads to high levels of differentiation. Our genetic variation explains some of this, 
but we are the least genetically diverse great ape-two groups of chimpanzees in the Congo are more genetically different from each other than two groups of humans plucked from Berlin and Beijing (Prado-Martinez et al. 2013). Most of our diversity is cultural rather than genetic (Bell, Richerson, and McElreath 2009; Muthukrishna et al. 2020); culture drives much of our withinspecies phenotypic variation. At best, genetic effects can only be specified within the ambit of a specific cultural context, but even the boundaries of that context cannot be specified without cultural evolutionary tools. And because culture is also evolving over time, these cultural contexts also require a timestamp. Heritability is not a property of a trait in itself, because in the absence of a reference culture it is necessarily unstable.

Cultural evolution yields cultural clusters. Within each society, environments are relatively homogenous, and the homogeneity that we find within societies is coupled with pronounced heterogeneity between societies (Bell, Richerson, and McElreath 2009; Richerson et al. 2016). Extrapolating genetic effects beyond a species is obviously mistaken, but so too is extrapolating a genetic effect beyond a culture. But this is what researchers have been doing since Galton, and it is ingrained in both our methodology and our thinking, culminating in the recent triumphalist discourse surrounding behavioral genetics and $\mathrm{GWAS}^{10}$. The movement toward more diverse genomic data ought to make some of these problems more obvious, just as more diverse psychological data made the problems of WEIRD psychology more obvious. But here too, data alone will not solve the problem (Muthukrishna et al. 2020; Muthukrishna and Henrich 2019). The question is not whether genes or culture contribute more to a behavioral trait, as behavioral traits can only be understood as emergent products of our dual inheritance, genetic and cultural. Nothing in behavioral genetics makes sense except in the light of cultural evolution.

\footnotetext{
${ }^{10}$ Some recent prominent examples that have entered the public discourse include Plomin (2019) and Murray (2020). Plomin states,

"Polygenic scores are the ultimate psychological test because, for the first time, they can tell our genetic fortunes." Similarly, Murray declares: "By the end of the 2020s, it will be widely accepted that quantitative studies of social behavior that don't use polygenic scores usually aren't worth reading. More formally, it will be widely accepted that the predictive validity of polygenic scores gives us useful information about causes even though we still don't understand the causal pathways."
} 


\section{APPENDIX: MODELING THE EFFECT OF CULTURAL EVOLUTION ON HERITABILITY}

\subsection{Variance partitioning model}

Here we describe a simple mathematical model that captures the relationship between cultural evolution and heritability. Cultural evolution is a process in which some cultural variants spread through a population more prolifically than others. This spread can be partly due to intrinsic differences in the trait (e.g., steel axes are better than stone axes) and partly due to social learning strategies like the conformist bias, success bias, and prestige bias (for summary, see Kendal et al. 2018; Chudek, Muthukrishna, and Henrich 2015). Such strategies vary in their rules for selecting what to learn or whom to learn from, but they all lead to the disproportionate adoption of particular cultural variants over others, and thus to a reduction in the population-level variability of behaviors. Individual incremental improvement, individual learning (Rogers 1988; Legare and Nielsen 2015), cultural transmission error, recombination of cultural traits, and other sources of innovation (Muthukrishna and Henrich 2016) will continue to inject novel variants into the population, but the fact that a nearly unrestricted number of learners can inherit the behaviors and ideas of a few influential individuals makes it easy for cultural transmission to induce homogeneity. It is not only behavioral traits that become similar within a population through cultural transmission, but also environmental factors that shape behavioral traits, such as nutrition, sanitation, education, and media.

Heritability is defined as the proportion of phenotypic variance for some trait that is explained by genes. For broad-sense heritability, $H^{2}=V_{G} / V_{P}$, where $H^{2}$ is heritability, $V_{P}$ is the variance in phenotype, and $V_{G}$ is the phenotypic variance explained by all genetic factors including additive genetic variance, dominance interactions, and epistatic interactions. Because total phenotypic variance is made up of contributions from both genes and environment $\left(V_{P}=V_{G}+V_{E}\right)$, a reduction in the environmental contribution necessarily increases heritability; cultural evolution has been a powerful force for behavioral homogenization and hence reduction in environmental variance over human history. Behavioral geneticists partition phenotypic variance into genetic and environmental components, but here we further partition the environmental component into 
environmental variation unaffected by cultural evolution (e.g., UV exposure due to geographic location) and environmental variation affected by cultural evolution (e.g., UV exposure due to sunscreen use). For convenience, we refer to the former as the ecological environmental variance component and the latter as the cultural environmental variance component, and represent this partition using the following notation:

$$
\text { (1) } V_{E}=V_{e}+V_{c}
$$

where $V_{e}$ and $V_{c}$ denote the phenotypic variance explained by ecology and culture, respectively. In reality, the cultural environment and even individual cultural traits will typically have a highdimensional variance structure, such as with the various components of a tool or a ritual that can be independently modified, but here we employ a unidimensional space of cultural variation. For simplicity, we model cultural environmental variation as a uniform continuous distribution that is bound by $k_{\text {min }}$, the most unfavorable environmental state (for some given phenotype) within the experienced range of environments, and $k_{\max }$, the most favorable. We can use the theoretically expected variance of the continuous uniform distribution $\left(\frac{1}{12}(b-a)^{2}\right.$, where $a$ and $b$ are the minimum and maximum values) to rewrite (1):

$$
\text { (2) } V_{E}=V_{e}+\frac{1}{12}\left(k_{\max }-k_{\min }\right)^{2}
$$

We can then substitute (2) into the standard formula for broad-sense heritability:

$$
\text { (3) } H^{2}=\frac{V_{G}}{V_{G}+V_{e}+\frac{1}{12}\left(k_{\max }-k_{\min }\right)^{2}}
$$

Heritability thus decreases when $\left(k_{\max }-k_{\min }\right)$ is large and increases when it is small. The smaller the experienced range of aspects of the cultural environment that contribute to phenotypic variation, the more phenotypic variation there is left to be explained by genes and by consequence, the higher the heritability. The magnitude of this cultural effect depends upon (i) the ratio of $V_{c}$ to $V_{e}$, which is the extent of cultural influence upon the environmental variance 
component as a whole, as well as (ii) the ratio of genetic influence to total environmental influence $\left(V_{G}\right.$ to $\left.V_{E}\right)$. We illustrate the effect of each of these variance components on heritability in Figure 4. To keep the model simple, we omit the gene-environment interaction and geneenvironment correlation terms.

This model shows how heritability can be shaped by a shifting cultural environment. Once again, this insight is in itself neither new nor surprising. But the model we describe here allows us to go further and consider the particular, systematic ways in which the cultural environment is expected to change. Although this model does not incorporate cultural dynamics as such, we can nonetheless map two broad cultural dynamics onto its framework: (1) new innovations elevating the upper bound of cultural complexity (Henrich 2004a; Muthukrishna and Henrich 2016) and (2) diffusion of these innovations (Rogers 2003; Henrich 2001; Comin and Hobijn 2010)—new traits emerge and then spread to fixation in the population. Within this framework, we can think of increases in cultural complexity as pushing up $k_{\text {max }}$, the most favorable cultural conditions in a society. In turn, we can think of diffusion as pushing up $k_{\text {min }}$, the most unfavorable cultural conditions in a society. As an example, imagine $k_{\max }$ is the educational contribution of the best school in a society and $k_{\min }$ is the educational contribution of the worst school in a society. In some societies, educational innovations diffuse quickly, whereas in others, there is more lag between the discovery of a new technology or pedagogical technique and its widespread adoption. Some societies are highly equal $\left(k_{\max }-k_{\min }\right.$ is small $)$ and others are more unequal $\left(k_{\max }-k_{\min }\right.$ is large). The magnitude of the lag between increasing $k_{\max }$ and $k_{\min }$-for example, how quickly educational innovations in the best schools diffuse to other schoolsimpacts environmental heterogeneity and thus heritability: we illustrate this effect as different trajectories of cultural change in Figure 4c. Moreover, by further decomposing $k_{\max }$ and $k_{m i n}$ into their governing cultural dynamics, we can predict the trajectories of heritability over time. 
(a)

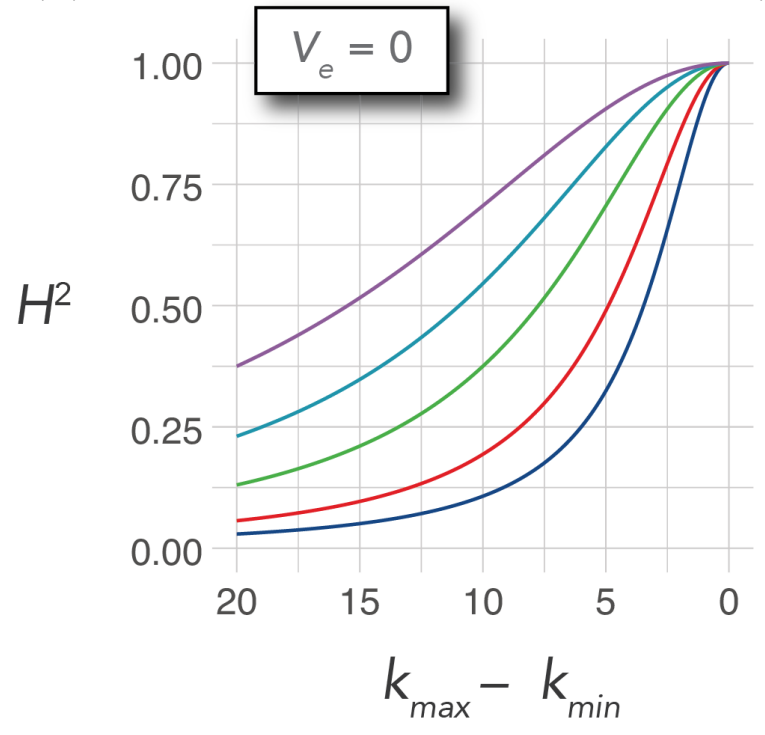

(b)

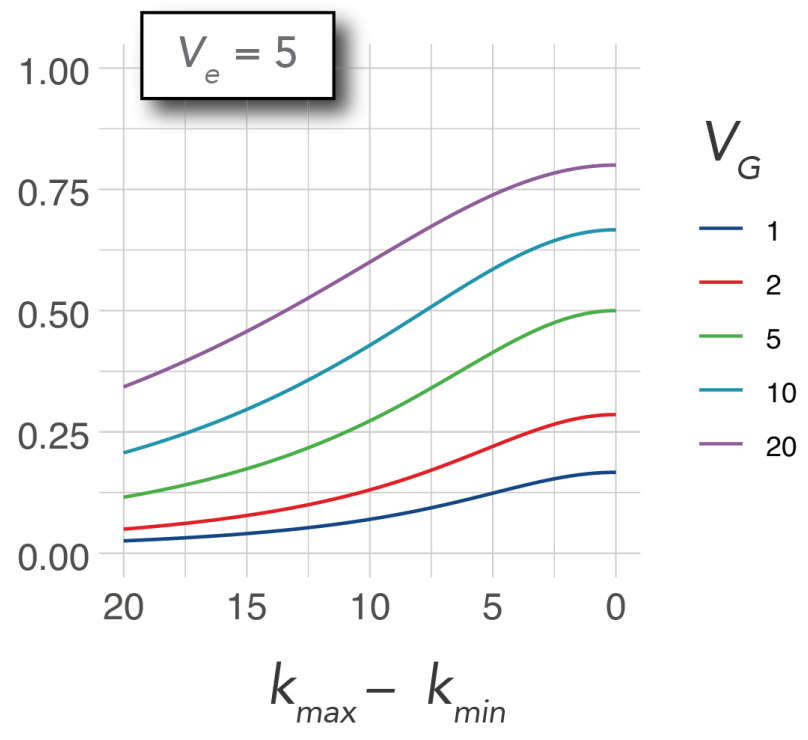

(c)

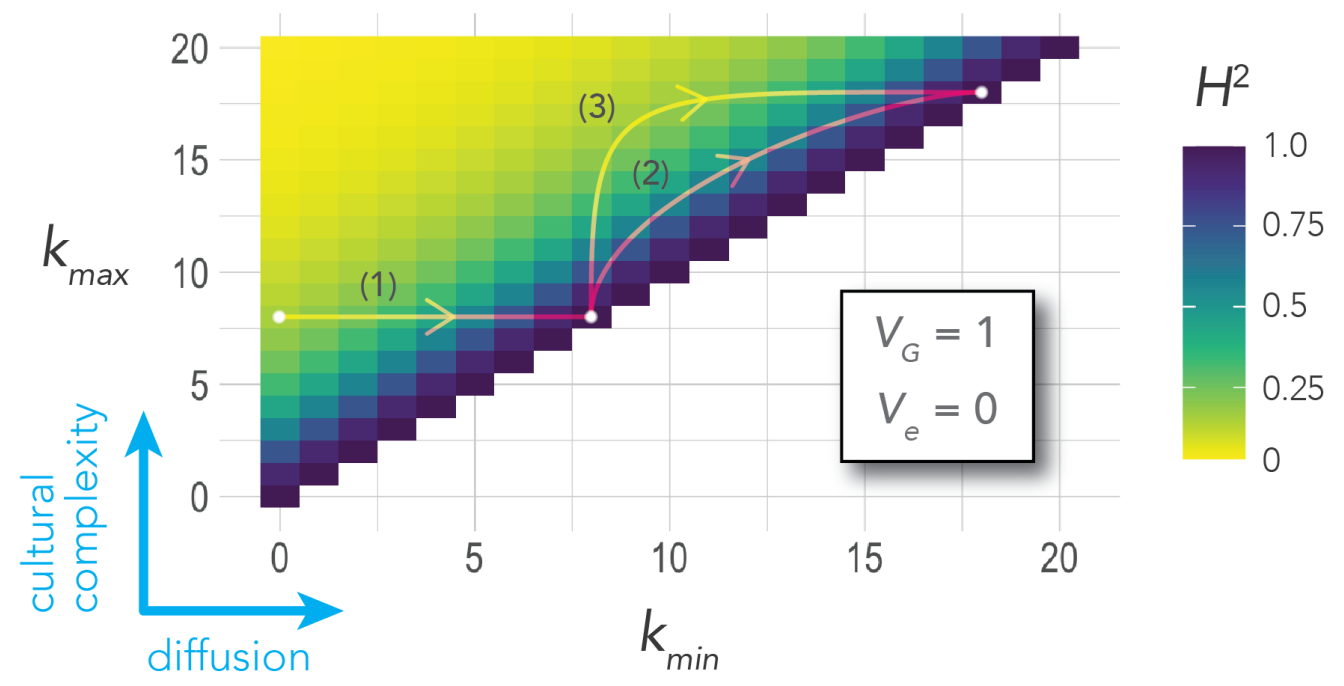

Figure 4: Visualizations of Equation 3. Heritability curves as a function of cultural range $\left(k_{\max }-k_{\min }\right)$ and of the amount of genetic variance $\left(V_{G}\right)$. (a) Values are computed for $V_{e}=0$ (the environment is entirely explained by cultural factors) and (b) for $V_{e}=5$ (some of the environment is explained by non-cultural factors, such as climate). (c) An alternative visualization in which we look at the absolute values of $k_{\max }$ and $k_{\min }$ rather than just their difference, plotted for $V_{e}=0$ and $V_{G}=1$. An increase in $k_{\text {max }}$ expands environmental variation and implies increasing maximum cultural complexity, whereas an increase in $k_{\text {min }}$ reduces environmental variation and implies diffusion. Trajectory 1 represents a society's transition from a more unequal cultural environment to a more equal cultural environment, but with no increase in cultural complexity. Trajectories 2 and 3 represent a simultaneous increase in cultural complexity and diffusion of the newly established complex traits, where a rising $k_{\max }$ pulls $k_{\min }$ upward but with varying lags: for trajectory 2 there is little lag between increase in the complexity of the culture and its spread, whereas for trajectory 3 there is considerable lag, with a longer period of relative cultural inequality. Genetic heritability decreases with rising cultural complexity and increases with cultural equality (diffusion). For example, continued innovation will reduce heritability up to the point at which the society is maximally unequal, and then increase heritability once more as the cultural innovations spread to the entire population-i.e., curves 2 and 3 are non-monotonic. 


\subsection{Cultural dynamics model}

To capture the effect of a changing cultural environment on heritability estimates over time, we construct a simple model that incorporates a time axis and whose parameters can be adjusted to compare different cultural trajectories. This dynamic model builds upon the previous variance partitioning model, but extends it by representing $k_{\min }$ and $k_{\max }$ - the upper and lower bounds of the acquired range of cultural complexity - as sigmoidal trajectories (s-shaped curves), which are commonly used to model cultural diffusion (Rogers 2003; Henrich 2001). Here we use the Gompertz function, which is a special case of the generalized logistic function that is commonly used in biology to model growth. In particular, we employ the following re-parameterization of the Gompertz that is given by Tjørve and Tjørve (2017):

$$
\begin{aligned}
& \text { (4) } \quad k_{\text {min }}=k_{0}\left(\frac{k_{0}}{A}\right)^{\exp \left(-e \beta_{k_{\text {min }}} t / A\right)-1} \\
& \text { (5) } \quad k_{\text {max }}=k_{0}\left(\frac{k_{0}}{A}\right)^{\exp \left(-e \beta_{k_{\text {max }}} t / A\right)-1}
\end{aligned}
$$

where $t$ is time; $A$ is the upper asymptote of both $k_{\min }$ and $k_{\max } ; k_{0}$ is a parameter that fixes both $k_{\min }$ and $k_{\max }$ to a particular value at $t=0$; and $\beta_{k_{\min }}$ and $\beta_{k_{\max }}$ are the maximum growth rates of $k_{\text {min }}$ and $k_{\max }$ respectively. At any given time point, $k_{\min } \leq k_{\max }$ by definition: this condition is satisfied for all $t \geq 0$ (but not for $t<0$ ) when we set $\beta_{k_{\max }}$ to be larger than $\beta_{k_{\text {min }}}$, due to both curves being fixed to the same value $k_{0}$ at $t=0$. This model therefore requires that $k_{\min }$ and $k_{\max }$ only be evaluated over non-negative time points.

The parameters $\beta_{k_{\min }}$ and $\beta_{k_{\max }}$ determine the shapes of the diffusion and innovation curves respectively. They indicate maximum (absolute) growth rates, which occur at the inflection points of $k_{\min }$ and $k_{\max }$. In a Gompertz function, inflection occurs at $\frac{1}{e}=36.79 \%$ of the upper asymptote value $A$, regardless of parameter values. Therefore, curves with a larger maximum growth rate are steeper and approach the asymptote more rapidly. By adjusting $\beta_{k_{\min }}$ and $\beta_{k_{\max }}$, we can model the variation in relative rates of cultural innovation and diffusion across different 
types of society, as well as the impact of these different cultural trajectories on heritability. Many factors can affect these rates of innovation and diffusion (Muthukrishna and Schaller 2020; Schaller and Muthukrishna in press; Muthukrishna and Henrich 2016; De, Nau, and Gelfand 2017; Gelfand 2018; Kolodny, Creanza, and Feldman 2015; Rogers 2003). For example, a "tight" society may have low rates of radical innovation, high rates of incremental innovation, and high rates of diffusion of these innovations, whereas a "loose" society may have high rates of radical innovation but lower rates of diffusion (Gelfand 2018). Other societies may be high or low in both. In Figure 5 we illustrate a $2 \times 2$ of societies that vary in their innovation and diffusion rates.

Heritability is computed using the same procedure as the variance partitioning model, but dynamically. Following equation (2), we compute the cultural variance component $V_{c}$ at time $t$ by taking the variance of the uniform distribution over the interval $\left[k_{\max }, k_{\max }\right]$ at time $t$. Phenotypic variance explained by the environment (i.e., ecology and culture) at time $t$ therefore takes the following form:

$$
V_{E(t)}=V_{e}+\frac{1}{12}\left(k_{\max (t)}-k_{\min (t)}\right)^{2}
$$

where $V_{e}$ is again the ecological variance component, which we treat as static compared to the rapidly changing cultural environment. By selecting values for both $V_{e}$ and $V_{G}$ (the genetic variance component), we can thus compute broad-sense heritability at time $t$ in the same manner as (3):

$$
\text { (7) } \quad H_{(t)}^{2}=\frac{V_{G}}{V_{G}+V_{e}+\frac{1}{12}\left(k_{\max (t)}-k_{\min (t)}\right)^{2}}
$$

These dynamics of changing environmental variance and heritability were only implicitly included in the variance partitioning model above (see Figure 4c) but are represented here explicitly. The effect of innovation and diffusion on cultural variance and heritability are illustrated in the lower panels of each society in Figure 5. These results show, for example, that 
societies with both rapid innovation and rapid diffusion of these innovations should experience large transient changes in heritability (Figure 5a), while a combination of slow innovation and rapid diffusion has little impact on heritability (Figure 5c). Societies with rapid innovation but long lags in diffusion will experience large changes in heritability over longer time scales (Figure $5 \mathrm{~b}$ ), as will societies with slow innovation and slow diffusion (Figure $5 \mathrm{~d}$ ). In reality, there may be multiple relevant cultural innovations for some given phenotypic trait at any given point in time- - under such a scenario, heritability would tend to fluctuate around some intermediate value rather than traverse the full range depicted in the lower panels of Figure 5. The output of this model predicts that on average, heritability of culturally transmissible traits should be higher in more homogenous (tight or less clustered) societies than in less homogeneous (loose or more clustered) societies, as discussed in Section 2.2.3. 
(a)

\section{Rapid Innovation \& Rapid Diffusion}
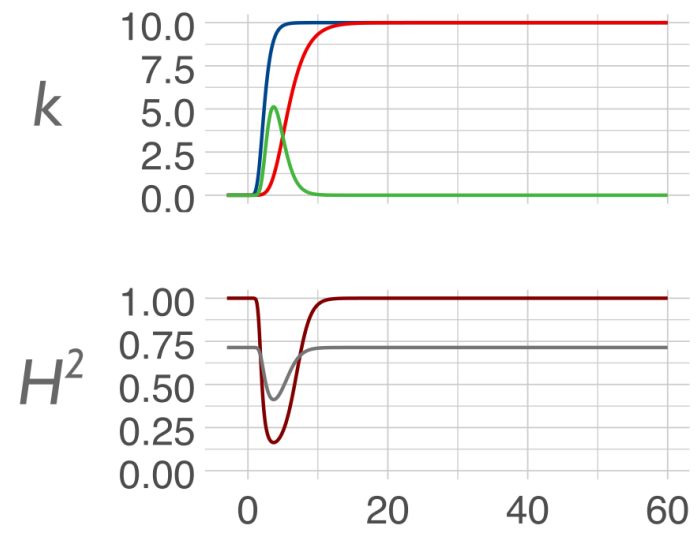

(c)
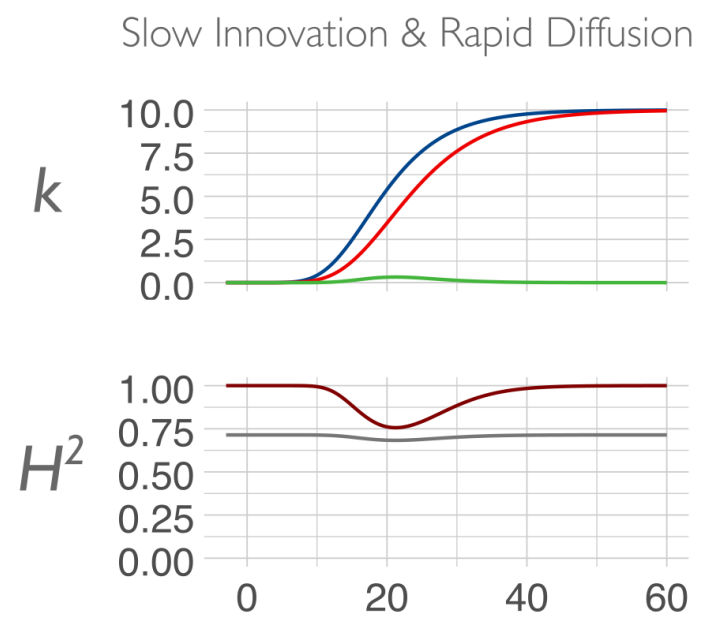

(b)

\section{Rapid Innovation \& Slow Diffusion}
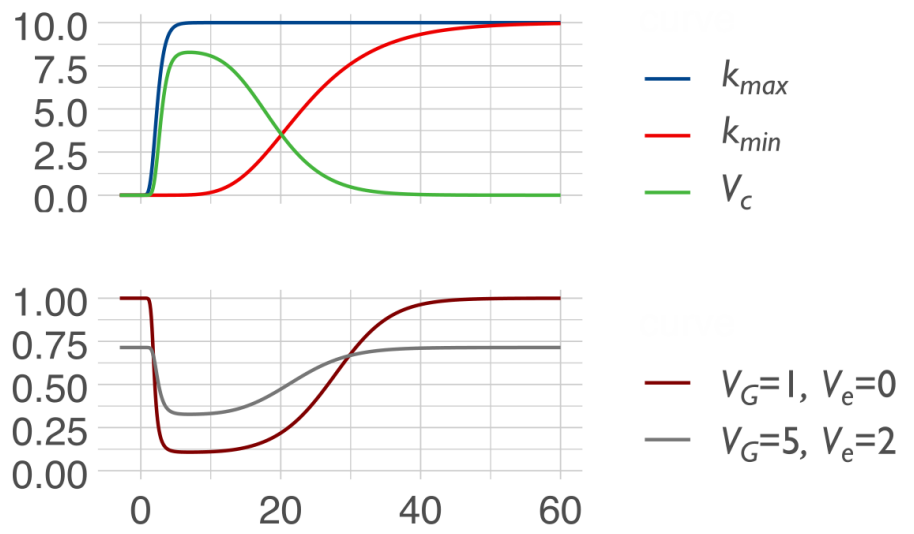

(d)

\section{Slow Innovation \& Slow Diffusion}
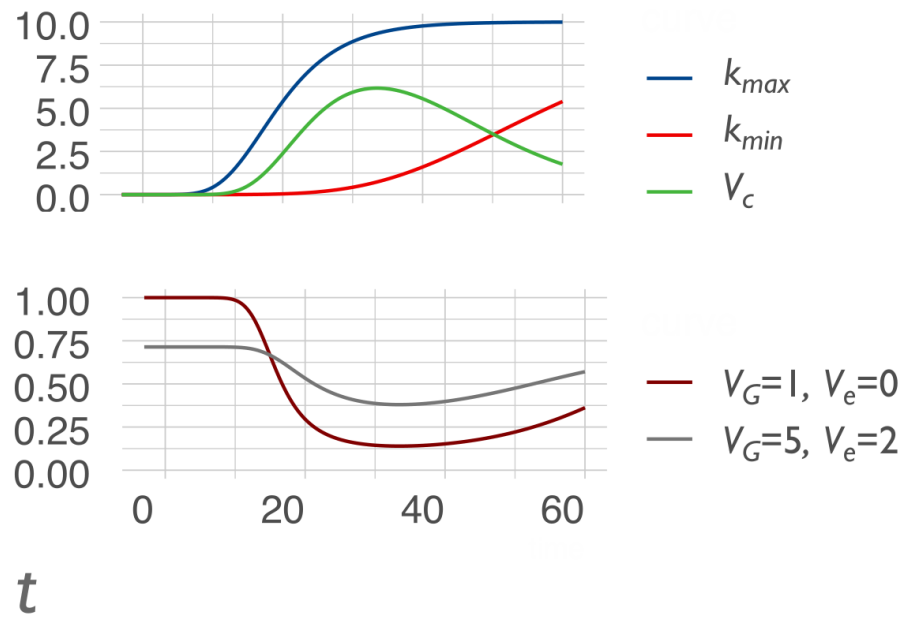

0.75

0.50

0.25

0.00

$t$

Figure 5: Output examples of the cultural dynamics model, for 4 prototypical societies. For each society, the upper panel displays the change over time in the lower $\left(k_{\min }\right)$ and upper bounds $\left(k_{\max }\right)$ of the society's cultural complexity as it pertains to some given phenotypic trait, together with the variance explained by the cultural environment $V_{c}$, which is the theoretically expected variance of the uniform distribution over the interval $\left[k_{\min }, k_{\max }\right]$. The lower panel displays the change in heritability over time under two different settings for genetic and ecological variance components. Global model parameters are set to $A=10$ and $W_{0}=10^{-6}$. (a) A society that innovates rapidly while also diffusing these innovations across the population rapidly; maximum growth rates of $k_{\min }$ and $k_{\max }$ are $\beta_{k_{\min }}=2$ and $\beta_{k_{\max }}=5$, respectively. (b) A society that innovates rapidly but whose innovations are slow to diffuse; $\beta_{k_{\min }}=0.5$ and $\beta_{k_{\max }}=5$. (c) A society that innovates only gradually but whose innovations diffuse quickly; $\beta_{k_{\min }}=0.5$ and $\beta_{k_{\max }}=0.6$. (d) A society that innovates only gradually and whose innovations take even longer to diffuse; $\beta_{k_{\min }}=0.2$ and $\beta_{k_{\text {max }}}=0.6$. 


\section{ACKNOWLEDGEMENTS}

We are grateful for comments to an earlier version of this target article from Hovig Artinian, Benjamin Cheung, Ilan Dar-Nimrod, Steven Heine, Joseph Henrich, and Jay Lasker, as well as for the valuable input by the anonymous reviewers. We thank all those who posted comments about our preprint on Twitter and other social media. We would also like to thank Veronika Plant for illustrating Figure 2, as well as Madison Johnson, Mercedes Lucia Minahan, Monty Nitschke, Haley P. Riggs, and Mona J. Xue for replicating an earlier version of our model.

\section{FUNDING STATEMENT}

This research received no specific grant from any funding agency, commercial or not-for-profit sectors.

\section{CONFLICTS OF INTEREST STATEMENT}

Conflicts of Interest: None.

\section{REFERENCES}

Abdellaoui, Abdel, David Hugh-Jones, Loic Yengo, Kathryn E. Kemper, Michel G. Nivard, Laura Veul, Yan Holtz, et al. 2019. 'Genetic Correlates of Social Stratification in Great Britain'. Nature Human Behaviour 3 (12): 1332-42. https://doi.org/10.1038/s41562-0190757-5.

Abdellaoui, Abdel, Karin J.H. Verweij, and Michel G. Nivard. 2021. 'Geographic Confounding in Genome-Wide Association Studies'. Preprint. bioRxiv. https://doi.org/10.1101/2021.03.18.435971. 
Aiello, Leslie C., and Peter Wheeler. 1995. 'The Expensive-Tissue Hypothesis: The Brain and the Digestive System in Human and Primate Evolution'. Current Anthropology 36 (2): 199221. https://doi.org/10.1086/204350.

Akiyama, Masato, Kazuyoshi Ishigaki, Saori Sakaue, Yukihide Momozawa, Momoko Horikoshi, Makoto Hirata, Koichi Matsuda, et al. 2019. 'Characterizing Rare and Low-Frequency Height-Associated Variants in the Japanese Population'. Nature Communications 10 (December): 4393. https://doi.org/10/ggnj6n.

Albalat, Ricard, and Cristian Cañestro. 2016. 'Evolution by Gene Loss'. Nature Reviews Genetics 17 (7): 379-91. https://doi.org/10.1038/nrg.2016.39.

Amici, Federica, Alex Sánchez-Amaro, Carla Sebastián-Enesco, Trix Cacchione, Matthias Allritz, Juan Salazar-Bonet, and Federico Rossano. 2019. 'The Word Order of Languages Predicts Native Speakers' Working Memory'. Scientific Reports 9 (1): 1124. https://doi.org/10.1038/s41598-018-37654-9.

Ammerman, Albert J., and L. L. Cavalli-Sforza. 1984. The Neolithic Transition and the Genetics of Populations in Europe. Princeton, N.J: Princeton University Press.

Anderson, Benedict. 2006. Imagined Communities: Reflections on the Origins and Spread of Nationalism. London: Verso.

Apicella, Coren L, Frank W Marlowe, James H Fowler, and Nicholas A Christakis. 2012. 'Social Networks and Cooperation in Hunter-Gatherers'. Nature 481 (7382): 497-501.

Apicella, Coren, Ara Norenzayan, and Joseph Henrich. 2020. 'Beyond WEIRD: A Review of the Last Decade and a Look Ahead to the Global Laboratory of the Future'. Evolution and Human Behavior, Beyond Weird, 41 (5): 319-29. https://doi.org/10.1016/j.evolhumbehav.2020.07.015.

Assary, Elham, John Paul Vincent, Robert Keers, and Michael Pluess. 2018. 'Gene-Environment Interaction and Psychiatric Disorders: Review and Future Directions'. Seminars in Cell Eซ Developmental Biology, Arc/ARg3.1, $77 \quad$ (May): 133-43. https://doi.org/10.1016/j.semcdb.2017.10.016.

Australian Institute of Health and Welfare. 2016. 'Skin Cancer in Australia.' https://www.aihw.gov.au/reports/cancer/skin-cancer-in-australia/contents/table-ofcontents. 
Aylin, Paul, Sara Morris, Jon Wakefield, Ana Grossinho, Lars Jarup, and Paul Elliott. 2001. 'Temperature, Housing, Deprivation and Their Relationship to Excess Winter Mortality in Great Britain, 1986-1996'. International Journal of Epidemiology 30 (5): 1100-1108. https://doi.org/10.1093/ije/30.5.1100.

Barrett, H. Clark. 2020. 'Deciding What to Observe: Thoughts for a Post-WEIRD Generation'. Evolution and Human Behavior, June. https://doi.org/10/ggzsjc.

Barrett, H. Clark, Alexander Bolyanatz, Alyssa N. Crittenden, Daniel M. T. Fessler, Simon Fitzpatrick, Michael Gurven, Joseph Henrich, et al. 2016. 'Small-Scale Societies Exhibit Fundamental Variation in the Role of Intentions in Moral Judgment'. Proceedings of the National Academy of Sciences 113 (17): 4688-93. https://doi.org/10.1073/pnas.1522070113.

Barsh, Gregory S. 2003. 'What Controls Variation in Human Skin Color?' PLoS Biology 1 (1): e27. https://doi.org/10.1371/journal.pbio.0000027.

Bates, Timothy C., Gary J. Lewis, and Alexander Weiss. 2013. 'Childhood Socioeconomic Status Amplifies Genetic Effects on Adult Intelligence'. Psychological Science 24 (10): 2111-16. https://doi.org/10.1177/0956797613488394.

Bathelt, Joe, Duncan Astle, Jessica Barnes, F. Lucy Raymond, and Kate Baker. 2016. 'Structural Brain Abnormalities in a Single Gene Disorder Associated with Epilepsy, Language Impairment and Intellectual Disability'. NeuroImage: Clinical 12 (February): 655-65. https://doi.org/10/f9f2ts.

Beam, Christopher R., Eric Turkheimer, William T. Dickens, and Deborah Winders Davis. 2015. 'Twin Differentiation of Cognitive Ability Through Phenotype to Environment Transmission: The Louisville Twin Study'. Behavior Genetics 45 (6): 622-34. https://doi.org/10.1007/s10519-015-9756-0.

Bell, Adrian V., Peter J. Richerson, and Richard McElreath. 2009. 'Culture Rather than Genes Provides Greater Scope for the Evolution of Large-Scale Human Prosociality'. Proceedings of the National Academy of Sciences 106 (42): 17671-74. https://doi.org/10/bq44jk.

Berg, Jeremy J, Arbel Harpak, Nasa Sinnott-Armstrong, Anja Moltke Joergensen, Hakhamanesh Mostafavi, Yair Field, Evan August Boyle, et al. 2019. 'Reduced Signal for Polygenic Adaptation of Height in UK Biobank'. ELife 8: e39725. https://doi.org/10.7554/eLife.39725. 
Bergen, Sarah E., Charles O. Gardner, and Kenneth S. Kendler. 2007. 'Age-Related Changes in Heritability of Behavioral Phenotypes Over Adolescence and Young Adulthood: A MetaAnalysis'. Twin Research and Human Genetics 10 (3): 423-33. https://doi.org/10/dm2q33.

Berry, John W. 1972. 'Radical Cultural Relativism and the Concept of Intelligence'. In Mental Tests and Cultural Adaptation, edited by LJ Cronbach and PJD Drenth, 77-88. The Hague: Mouton.

Bitarello, Bárbara D., and Iain Mathieson. 2020. 'Polygenic Scores for Height in Admixed Populations'. G3: Genes|Genomes|Genetics 10 (11): 4027. https://doi.org/10/gjrsrm.

Bloom, Nicholas. 2020. 'Are Ideas Getting Harder to Find?' American Economic Review 110 (4): 1104-44. https://doi.org/10.1257/aer.20180338.

Bolger, Donald J., Charles A. Perfetti, and Walter Schneider. 2005. 'Cross-Cultural Effect on the Brain Revisited: Universal Structures plus Writing System Variation'. Human Brain Mapping 25 (1): 92-104. https://doi.org/10/fdvnkc.

Bordenstein, Seth R., and Kevin R. Theis. 2015. 'Host Biology in Light of the Microbiome: Ten Principles of Holobionts and Hologenomes'. PLOS Biology 13 (8): e1002226. https://doi.org/10.1371/journal.pbio.1002226.

Bouchard, Thomas J. 2009. 'Genetic Influence on Human Intelligence (Spearman's $g$ ): How Much?' Annals of Human Biology 36 (5): 527-44. https://doi.org/10.1080/03014460903103939.

— 2013. 'The Wilson Effect: The Increase in Heritability of IQWith Age'. Twin Research and Human Genetics 16 (5): 923-30. https://doi.org/10.1017/thg.2013.54.

Boyd, Robert. 2018. A Different Kind of Animal: How Culture Transformed Our Species. The University Center for Human Values Series. Princeton: Princeton University Press.

Boyd, Robert, and Peter J. Richerson. 1985. Culture and the Evolutionary Process. University of Chicago Press.

—. 1987. 'The Evolution of Ethnic Markers'. Cultural Antbropology 2 (1): 65-79. https://doi.org/10/fv9vnv.

Boyle, Evan A., Yang I. Li, and Jonathan K. Pritchard. 2017. 'An Expanded View of Complex Traits: From Polygenic to Omnigenic'. Cell 169 (7): 1177-86. https://doi.org/10/gcpgdz. 
Brandt, Mark J., and Geoffrey A. Wetherell. 2012. 'What Attitudes Are Moral Attitudes? The Case of Attitude Heritability'. Social Psychological and Personality Science 3 (2): 172-79. https://doi.org/10.1177/1948550611412793.

Bratsberg, Bernt, and Ole Rogeberg. 2018. 'Flynn Effect and Its Reversal Are Both Environmentally Caused'. Proceedings of the National Academy of Sciences 115 (26): 6674-78. https://doi.org/10.1073/pnas.1718793115.

Briley, Daniel A., K. Paige Harden, and Elliot M. Tucker-Drob. 2015. 'Genotype $\times$ Cohort Interaction on Completed Fertility and Age at First Birth'. Behavior Genetics 45 (1): 7183. https://doi.org/10/f6zwc8.

Brinch, C. N., and T. a. Galloway. 2012. 'Schooling in Adolescence Raises IQ Scores'. Proceedings of the National Academy of Sciences 109: 425-30. https://doi.org/10/fx74wv.

Bronfenbrenner, Urie, and Stephen J Ceci. 1994. 'Nature-Nurture Reconceptualized in Developmental Perspective: A Bioecological Model'. Psychological Review 101 (4): 568-86. https://doi.org/10.1037/0033-295X.101.4.568.

Brumpton, Ben, Eleanor Sanderson, Fernando Pires Hartwig, Sean Harrison, Gunnhild Åberge Vie, Yoonsu Cho, Laura D Howe, et al. 2020. 'Avoiding Dynastic, Assortative Mating, and Population Stratification Biases in Mendelian Randomization through within-Family Analyses'. Nature Communications 11 (3519). https://doi.org/10.1101/602516.

Brustad, M, T Sandanger, L Aksnes, and E Lund. 2004. 'Vitamin D Status in a Rural Population of Northern Norway with High Fish Liver Consumption'. Public Health Nutrition 7 (6): 783-89. https://doi.org/10.1079/PHN2004605.

Byars, Sean G, Douglas Ewbank, Diddahally R Govindaraju, and Stephen C Stearns. 2010. 'Natural Selection in a Contemporary Human Population' 107: 1787-92. https://doi.org/10.1073/pnas.0906199106.

Carter, C. J. 2013. 'Toxoplasmosis and Polygenic Disease Susceptibility Genes: Extensive Toxoplasma Gondii Host/Pathogen Interactome Enrichment in Nine Psychiatric or Neurological Disorders'. Journal of Pathogens 2013: 1-29. https://doi.org/10.1155/2013/965046.

Cashman, Kevin D, Kirsten G Dowling, Zuzana Škrabáková, Marcela Gonzalez-Gross, Jara Valtueña, Stefaan De Henauw, Luis Moreno, et al. 2016. 'Vitamin D Deficiency in 
Europe: Pandemic?' The American Journal of Clinical Nutrition 103 (4): 1033-44. https://doi.org/10.3945/ajcn.115.120873.

Cavalli-Sforza, L., M. Feldman, K. Chen, and S. Dornbusch. 1982. 'Theory and Observation in Cultural Transmission'. $\quad$ Science $218 \quad$ (4567): 19-27. https://doi.org/10.1126/science.7123211.

Cavalli-Sforza, L. L. 2001. Genes, Peoples, and Languages. Berkeley: University of California Press.

Cavalli-Sforza, L L, and M W Feldman. 1973. 'Cultural versus Biological Inheritance: Phenotypic Transmission from Parents to Children. (A Theory of the Effect of Parental Phenotypes on Children's Phenotypes).' American Journal of Human Genetics 25 (6): 618-37.

Cavalli-Sforza, L. L., A. Piazza, P. Menozzi, and J. Mountain. 1988. 'Reconstruction of Human Evolution: Bringing Together Genetic, Archaeological, and Linguistic Data'. Proceedings of the National Academy of Sciences 85 (16): 6002-6. https://doi.org/10.1073/pnas.85.16.6002.

Cavalli-Sforza, Luigi L, and Marcus W Feldman. 1981. Cultural Transmission and Evolution: $A$ Quantitative Approach. Princeton University Press. https://press.princeton.edu/titles/4409.html.

Cavalli-Sforza, Luigi Luca, Paolo Menozzi, and Alberto Piazza. 1994. The History and Geography of Human Genes. Princeton, NJ: Princeton University Press.

Ceci, Stephen J. 1991. 'How Much Does Schooling Influence General Intelligence and Its Cognitive Components? A Reassessment of the Evidence'. Developmental Psychology 27 (5): 703-22. https://doi.org/10.1037/0012-1649.27.5.703.

Chapuy, M.-C., P. Preziosi, M. Maamer, S. Arnaud, P. Galan, S. Hercberg, and P.J. Meunier. 1997. 'Prevalence of Vitamin D Insufficiency in an Adult Normal Population'. Osteoporosis International 7 (5): 439-43. https://doi.org/10.1007/s001980050030.

Charmantier, Anne, and Dany Garant. 2005. 'Environmental Quality and Evolutionary Potential: Lessons from Wild Populations'. Proceedings of the Royal Society B: Biological Sciences 272 (1571): 1415-25. https://doi.org/10/dn4hck.

Chater, N., F. Reali, and M. H. Christiansen. 2009. 'Restrictions on Biological Adaptation in Language Evolution'. Proceedings of the National Academy of Sciences 106 (4): 1015-20. https://doi.org/10.1073/pnas.0807191106. 
Chatterjee, I. B. 1973. 'Evolution and the Biosynthesis of Ascorbic Acid'. Science 182 (4118): 1271-72. https://doi.org/10.1126/science.182.4118.1271.

Chetty, Raj, and Nathaniel Hendren. 2018a. 'The Impacts of Neighborhoods on Intergenerational Mobility I: Childhood Exposure Effects'. The Quarterly Journal of Economics 133 (3): 110762. https://doi.org/10.1093/qje/qjy007.

— 2018b. 'The Impacts of Neighborhoods on Intergenerational Mobility II: County-Level Estimates'. The Quarterly Journal of Economics 133 (3): 1163-1228. https://doi.org/10.1093/qje/qjy006.

Christiansen, Morten H. 1994. 'Infinite Languages, Finite Minds: Connectionism, Learning and Linguistic Structure'. University of Edinburgh.

Christiansen, Morten H., and Nick Chater. 2008. 'Language as Shaped by the Brain'. Behavioral and Brain Sciences 31 (5): 489-509. https://doi.org/10/bpnv67.

Chudek, M, Michael Muthukrishna, and J Henrich. 2015. 'Cultural Evolution'. In The Handbook of Evolutionary Psychology, 2nd ed. Hoboken, NJ: John Wiley and Sons. https://doi.org/10.1002/9781119125563.evpsych230.

Chudek, Maciej, and Joseph Henrich. 2011. 'Culture-Gene Coevolution, Norm-Psychology and the Emergence of Human Prosociality'. Trends in Cognitive Sciences 15 (5): 218-26. https://doi.org/10.1016/j.tics.2011.03.003.

Clark, Cameron M., Linette Lawlor-Savage, and Vina M. Goghari. 2016. 'The Flynn Effect: A Quantitative Commentary on Modernity and Human Intelligence'. Measurement: Interdisciplinary Research and Perspectives 14 (2): 39-53. https://doi.org/10.1080/15366367.2016.1156910.

Cole, Michael. 1998. Cultural Psychology: A Once and Future Discipline. Cambridge, MA: Harvard University Press.

Coleman, Jonathan R. I., Julien Bryois, Héléna A. Gaspar, Philip R. Jansen, Jeanne E. Savage, Nathan Skene, Robert Plomin, et al. 2019. 'Biological Annotation of Genetic Loci Associated with Intelligence in a Meta-Analysis of 87,740 Individuals'. Molecular Psychiatry 24 (2): 182-97. https://doi.org/10/gd8h7x.

Colodro-Conde, Lucía, Frühling Rijsdijk, María J. Tornero-Gómez, Juan F. Sánchez-Romera, and Juan R. Ordoñana. 2015. 'Equality in Educational Policy and the Heritability of 
Educational Attainment'. Edited by Nikolaos Georgantzis. PLOS ONE 10 (11): e0143796. https://doi.org/10.1371/journal.pone.0143796.

Comin, Diego, and Bart Hobijn. 2010. 'An Exploration of Technology Diffusion'. American Economic Review 100 (5): 2031-59. https://doi.org/10.1257/aer.100.5.2031.

Cordain, Loren, S Boyd Eaton, Anthony Sebastian, Neil Mann, Staffan Lindeberg, Bruce A Watkins, James H O'Keefe, and Janette Brand-Miller. 2005. 'Origins and Evolution of the Western Diet: Health Implications for the 21st Century'. The American Journal of Clinical Nutrition 81 (2): 341-54. https://doi.org/10/gf9rzs.

Crawford, Nicholas G., Derek E. Kelly, Matthew E. B. Hansen, Marcia H. Beltrame, Shaohua Fan, Shanna L. Bowman, Ethan Jewett, et al. 2017. 'Loci Associated with Skin Pigmentation Identified in African Populations'. Science 358 (6365): eaan8433. https://doi.org/10.1126/science.aan8433.

Creanza, Nicole, Oren Kolodny, and Marcus W. Feldman. 2017. 'Cultural Evolutionary Theory: How Culture Evolves and Why It Matters'. Proceedings of the National Academy of Sciences 114 (30): 7782-89. https://doi.org/10/gfzkn3.

Crispo, Erika. 2007. 'Baldwin Effect and Genetic Assimilation: Revisiting Two Mechanisms of Evolutionary Change Mediated by Phenotypic Plasticity'. Evolution 61 (11): 2469-79. https://doi.org/10.1111/j.1558-5646.2007.00203.x.

Curtis, David. 2018. 'Polygenic Risk Score for Schizophrenia Is More Strongly Associated with Ancestry than with Schizophrenia': Psychiatric Genetics 28 (5): 85-89. https://doi.org/10/ggsp2z.

Dai, Chengzhen L., Mohammad M. Vazifeh, Chen-Hsiang Yeang, Remi Tachet, R. Spencer Wells, Miguel G. Vilar, Mark J. Daly, Carlo Ratti, and Alicia R. Martin. 2020. 'Population Histories of the United States Revealed through Fine-Scale Migration and Haplotype Analysis'. The American Journal of Human Genetics 106 (3): 371-88. https://doi.org/10/ghng3h.

Dar-Nimrod, Ilan, and Steven J. Heine. 2011. 'Genetic Essentialism: On the Deceptive Determinism of DNA.' Psychological Bulletin 137 (5): 800-818. https://doi.org/10.1037/a0021860. 
Davie, Gabrielle S, Michael G Baker, Simon Hales, and John B Carlin. 2007. 'Trends and Determinants of Excess Winter Mortality in New Zealand: 1980 to 2000'. BMC Public Health 7 (1): 263. https://doi.org/10.1186/1471-2458-7-263.

Davies, Gail, Max Lam, Sarah E. Harris, Joey W. Trampush, Michelle Luciano, W. David Hill, Saskia P. Hagenaars, et al. 2018. 'Study of 300,486 Individuals Identifies 148 Independent Genetic Loci Influencing General Cognitive Function'. Nature Communications 9 (1): 2098. https://doi.org/10.1038/s41467-018-04362-x.

Davies, Jamie A. 2014. Life Unfolding: How the Human Body Creates Itself. First Edition. Oxford, United Kingdom ; New York, NY, United States of America: Oxford University Press.

Davis, Helen Elizabeth. 2014. 'Variable Education Exposure and Cognitive Task Performance among the Tsiman' e Forager-Horticulturalists'. Albuquerque: University of New Mexico. https://digitalrepository.unm.edu/anth_etds/17.

Davis, Helen, Jonathan Stieglitz, Hillard Kaplan, and Michael Gurven. 2020. 'Poor School Quality Augments Differences in Children's Abstract Reasoning over Time: A Natural Experiment.' PsyArXiv. https://doi.org/10/ghn3fp.

De, Soham, Dana S. Nau, and Michele J. Gelfand. 2017. 'Understanding Norm Change: An Evolutionary Game-Theoretic Approach'. In Proceedings of the 16th Conference on Autonomous Agents and MultiAgent Systems, 1433-41. AAMAS '17. International Foundation for Autonomous Agents and Multiagent Systems.

Deacon, Terrence W. 1997. The Symbolic Species: The Co-Evolution of Language and the Brain. New York: WW Norton.

- 2003. 'Multilevel Selection in a Complex Adaptive System: The Problem of Language Origins'. In Evolution and Learning: The Baldwin Effect Reconsidered, edited by B. H. Weber and D. J. Depew, 81-106. Cambridge, MA: MIT Press.

Dean, Lewis G., Gill L. Vale, Kevin N. Laland, Emma Flynn, and Rachel L. Kendal. 2014. 'Human Cumulative Culture: A Comparative Perspective'. Biological Revierws 89 (2): 284301. https://doi.org/10.1111/brv.12053.

Dediu, D., and D. R. Ladd. 2007. 'Linguistic Tone Is Related to the Population Frequency of the Adaptive Haplogroups of Two Brain Size Genes, ASPM and Microcephalin'. Proceedings 
of the National Academy of Sciences 104 (26): 10944-49. https://doi.org/10.1073/pnas.0610848104.

Dediu, Dan. 2011. 'Are Languages Really Independent from Genes? If Not, What Would a Genetic Bias Affecting Language Diversity Look Like?' Human Biology 83 (2): 279-96. https://doi.org/10.3378/027.083.0208.

Dehaene, Stanislas, Véronique Izard, Elizabeth Spelke, and Pierre Pica. 2008. 'Log or Linear? Distinct Intuitions of the Number Scale in Western and Amazonian Indigene Cultures'. Science 320 (5880): 1217-20. https://doi.org/10/crp2tc.

Derex, Maxime, and Alex Mesoudi. 2020. 'Cumulative Cultural Evolution within Evolving Population Structures'. Trends in Cognitive Sciences 24 (8): 654-67. https://doi.org/10.1016/j.tics.2020.04.005.

Dias, Cristina, Sara B. Estruch, Sarah A. Graham, Jeremy McRae, Stephen J. Sawiak, Jane A. Hurst, Shelagh K. Joss, et al. 2016. 'BCL11A Haploinsufficiency Causes an Intellectual Disability Syndrome and Dysregulates Transcription'. The American Journal of Human Genetics 99 (2): 253-74. https://doi.org/10/f8zgjk.

Dickens, William T, and James R Flynn. 2001. 'Heritability Estimates Versus Large Environmental Effects: The IQParadox Resolved'. Psychological Review 108 (2): 346-69. https://doi.org/10/d88rxq.

Domahs, Frank, Korbinian Moeller, Stefan Huber, Klaus Willmes, and Hans-Christoph Nuerk. 2010. ‘Embodied Numerosity: Implicit Hand-Based Representations Influence Symbolic Number Processing across Cultures'. Cognition 116 (2): 251-66. https://doi.org/10.1016/j.cognition.2010.05.007.

Drouin, Guy, Jean-Remi Godin, and Benoit Page. 2011. 'The Genetics of Vitamin C Loss in $\begin{array}{lllll}\text { Vertebrates'. } \quad \text { Current } & \text { Genomics } & 12 & \text { (5): } & \text { 371-78. }\end{array}$ https://doi.org/10.2174/138920211796429736.

Duncan, L., H. Shen, B. Gelaye, J. Meijsen, K. Ressler, M. Feldman, R. Peterson, and B. Domingue. 2019. 'Analysis of Polygenic Risk Score Usage and Performance in Diverse Human Populations'. Nature Communications 10 (1): 3328. https://doi.org/10/ggkwt8. 
Dutton, Edward, Dimitri van der Linden, and Richard Lynn. 2016. 'The Negative Flynn Effect: A Systematic Literature Review'. Intelligence 59 (November): 163-69. https://doi.org/10.1016/j.intell.2016.10.002.

Ebbesen, Christian Laut. 2020. 'Flawed Estimates of Cognitive Ability in Clark et al. Psychological Science, 2020'. Preprint. PsyArXiv. https://doi.org/10.31234/osf.io/tzr8c.

Edlich, Richard F., Shelley S. Mason, Margot E. Chase, Allyson L. Fisher, K. Dean Gubler, William B. Long III, Jerry D. Giesy, and Marni L. Foley. 2009. 'Scientific Documentation of the Relationship of Vitamin D Deficiency and the Development of Cancer'. Journal of Environmental Pathology, Toxicology and Oncology 28 (2): 133-41. https://doi.org/10/ggrh6s.

Efferson, Charles, Sonja Vogt, and Ernst Fehr. 2020. 'The Promise and the Peril of Using Social Influence to Reverse Harmful Traditions'. Nature Human BehavIour 4: 55-68.

Elguero, Eric, Lucrèce M. Délicat-Loembet, Virginie Rougeron, Céline Arnathau, Benjamin Roche, Pierre Becquart, Jean-Paul Gonzalez, et al. 2015. 'Malaria Continues to Select for Sickle Cell Trait in Central Africa'. Proceedings of the National Academy of Sciences 112 (22): 7051-54. https://doi.org/10/f7fv49.

Emmons, Scott, Stephen Kobourov, Mike Gallant, and Katy Börner. 2016. 'Analysis of Network Clustering Algorithms and Cluster Quality Metrics at Scale'. PLOS ONE 11 (7): e0159161. https://doi.org/10/gbsh8d.

Engzell, Per, and Felix C. Tropf. 2019. 'Heritability of Education Rises with Intergenerational Mobility'. Proceedings of the National Academy of Sciences 116 (51): 25386-88. https://doi.org/10.1073/pnas.1912998116.

Enquist, Magnus, Pontus Strimling, Kimmo Eriksson, Kevin N. Laland, and Jonas Sjostrand. 2010a. 'One Cultural Parent Makes No Culture'. Animal Behaviour 79 (6): 1353-62. https://doi.org/10/b9zwhp.

Enquist, Magnus, Pontus Strimling, Kimmo Eriksson, Kevin Laland, and Jonas Sjostrand. 2010b. 'One Cultural Parent Makes No Culture'. Animal Behaviour 79 (6): 1353-62. https://doi.org/10.1016/j.anbehav.2010.03.009. 
European Statistical Office. 2020. 'Estimated Average Age of Young People Leaving the Parental Household by Sex'. https://ec.europa.eu/eurostat/en/web/products-datasets//YTH_DEMO_030.

Fadista, João, Alisa K Manning, Jose C Florez, and Leif Groop. 2016. 'The (in)Famous GWAS P-Value Threshold Revisited and Updated for Low-Frequency Variants'. European Journal of Human Genetics 24 (8): 1202-5. https://doi.org/10/f3pjts.

Fearon, J.D. 2003. 'Ethnic and Cultural Diversity by Country'. Journal of Economic Growth 8: 195222. https://doi.org/10/c2hmj2.

Fehr, Ernst, and Urs Fischbacher. 2003. 'The Nature of Human Altruism'. Nature 425 (6960): 785-91. https://doi.org/10.1038/nature02043.

—. 2004. 'Social Norms and Human Cooperation'. Trends in Cognitive Sciences 8 (4): 18590. https://doi.org/10.1016/j.tics.2004.02.007.

Feldman, Marcus, and Richard C Lewontin. 1975. 'The Heritability Hang-Up'. Science 190 (4220): 1163-68. https://doi.org/10.1126/science.1198102.

Feldman, Marcus W., and Sohini Ramachandran. 2018. 'Missing Compared to What? Revisiting Heritability, Genes and Culture'. Philosophical Transactions of the Royal Society B: Biological Sciences 373 (1743): 20170064. https://doi.org/10/gfgjwg.

Finlay, Barbara L, and Ryutaro Uchiyama. 2020. 'The Timing of Brain Maturation, Early Experience, and the Human Social Niche'. In Evolutionary Neuroscience, Second Edition, edited by Jon Kaas, 815-43. Academic Press.

Firth, Joseph, John Torous, Brendon Stubbs, Josh A. Firth, Genevieve Z. Steiner, Lee Smith, Mario Alvarez-Jimenez, et al. 2019. 'The "Online Brain”: How the Internet May Be Changing Our Cognition'. World Psychiatry 18 (2): 119-29. https://doi.org/10/gf67jq.

Fisher, Ronald A. 1952. 'Statistical Methods in Genetics'. Heredity 6: 1-12. https://doi.org/10.1038/hdy.1952.1.

Flynn, James R. 1984. 'The Mean IQ of Americans: Massive Gains 1932 to 1978.' Psychological Bulletin 95 (1): 29. https://doi.org/10/b6mh4g.

—. 1987. 'Massive IQ Gains in 14 Nations: What IQ Tests Really Measure.' Psychological Bulletin 101 (2): 171. https://doi.org/10/d78t3g. 
- 2007. What Is Intelligence?: Beyond the Flynn Effect. Cambridge University Press. https://doi.org/10.1017/CBO9780511605253.

Flynn, James R. 2009. 'The WAIS-III and WAIS-IV: Daubert Motions Favor the Certainly False over the Approximately True'. Applied Neuropsychology 16 (2): 98-104. https://doi.org/10.1080/09084280902864360.

Fogarty, Laurel, Nicole Creanza, and Marcus W. Feldman. 2019. 'The Life History of Learning: Demographic Structure Changes Cultural Outcomes'. Edited by Stefano Allesina. PLOS Computational Biology 15 (4): e1006821. https://doi.org/10.1371/journal.pcbi.1006821.

Fort, J. 2012. 'Synthesis between Demic and Cultural Diffusion in the Neolithic Transition in Europe'. Proceedings of the National Academy of Sciences 109 (46): 18669-73. https://doi.org/10.1073/pnas.1200662109.

Fowler, Tom, Rosamund J. Southgate, Thomas Waite, Ruth Harrell, Sari Kovats, Angie Bone, Yvonne Doyle, and Virginia Murray. 2015. 'Excess Winter Deaths in Europe: A MultiCountry Descriptive Analysis'. European Journal of Public Health 25 (2): 339-45. https://doi.org/10.1093/eurpub/cku073.

Freund, J., A. M. Brandmaier, L. Lewejohann, I. Kirste, M. Kritzler, A. Kruger, N. Sachser, U. Lindenberger, and G. Kempermann. 2013. 'Emergence of Individuality in Genetically Identical Mice'. Science 340 (6133): 756-59. https://doi.org/10.1126/science.1235294.

Freund, J., A.M. Brandmaier, L. Lewejohann, I. Kirste, M. Kritzler, A. Krüger, N. Sachser, U. Lindenberger, and G. Kempermann. 2015. 'Association between Exploratory Activity and Social Individuality in Genetically Identical Mice Living in the Same Enriched $\begin{array}{llll}\text { Environment'. } & \text { Neuroscience } & 309 & \text { (November): }\end{array}$ https://doi.org/10.1016/j.neuroscience.2015.05.027.

Fu, Qiaomei, Cosimo Posth, Mateja Hajdinjak, Martin Petr, Swapan Mallick, Daniel Fernandes, Anja Furtwängler, et al. 2016. 'The Genetic History of Ice Age Europe'. Nature 534 (7606): 200-205. https://doi.org/10.1038/nature17993.

Galton, Francis. 1869. Hereditary Genius. London: Macmillan.

- 1874. English Men of Science: Their Nature and Nurture. London: Macmillan.

$\begin{array}{llll}\text { Gapminder. } 2020 . & \text { 'Child }\end{array}$ https://www.gapminder.org/data/documentation/gd005/. 
Garland, Cedric F., Frank C. Garland, Edward D. Gorham, Martin Lipkin, Harold Newmark, Sharif B. Mohr, and Michael F. Holick. 2006. 'The Role of Vitamin D in Cancer Prevention'. American Journal of Public Health 96 (2): 252-61. https://doi.org/10/dmtr95.

Gelfand, Michele J. 2018. Rule Makers, Rule Breakers: How Tight and Loose Cultures Wire Our World. New York: Scribner.

Gelfand, Michele J., Lisa H. Nishii, and Jana L. Raver. 2006. 'On the Nature and Importance of Cultural Tightness-Looseness.' Journal of Applied Psychology 91 (6): 1225-44. https://doi.org/10.1037/0021-9010.91.6.1225.

Gelfand, Michele J., Jana L. Raver, Lisa Nishii, Lisa M. Leslie, Janetta Lun, Beng Chong Lim, Lili Duan, et al. 2011. 'Differences Between Tight and Loose Cultures: A 33-Nation Study'. Science 332 (6033): 1100-1104. https://doi.org/10.1126/science.1197754.

Giangrande, Evan J., Christopher R. Beam, Sarah Carroll, Lucas J. Matthews, Deborah W. Davis, Deborah Finkel, and Eric Turkheimer. 2019. 'Multivariate Analysis of the Scarr-Rowe Interaction across Middle Childhood and Early Adolescence'. Intelligence 77 (November): 101400. https://doi.org/10/ggxwrj.

Gibson, James J. 1979. The Ecological Approach to Visual Perception. Houghton Mifflin. https://doi.org/10.2307/1574154.

Gopnik, Alison, Clark Glymour, David M. Sobel, Laura E. Schulz, Tamar Kushnir, and David Danks. 2004. 'A Theory of Causal Learning in Children: Causal Maps and Bayes Nets.' Psychological Review 111 (1): 3-32. https://doi.org/10.1037/0033-295X.111.1.3.

Gordon, Robert. 2018. 'Why Has Economic Growth Slowed When Innovation Appears to Be Accelerating?' w24554. Cambridge, MA: National Bureau of Economic Research. https://doi.org/10.3386/w24554.

Gottlieb, Gilbert. 2003. 'On Making Behavioral Genetics Truly Developmental'. Human Development 46 (6): 337-55. https://doi.org/10.1159/000073306.

Greenfield, P. M. 2009. 'Technology and Informal Education: What Is Taught, What Is Learned'. Science 323 (5910): 69-71. https://doi.org/10.1126/science.1167190.

Greenfield, Patricia M. 1998. 'The Cultural Evolution of IQ'. In The Rising Curve: Long-Term Gains in IQ and Related Measures, edited by Ulric Neisser, 81-123. Washington DC: American Psychological Association. https://doi.org/10.1037/10270-003 
Groot, T. de, T. Vos, R. J. M. J. Vogels, and W. D. van Driel. 2013. 'Quality and Reliability in Solid-State Lighting'. In Solid State Lighting Reliability: Components to System, edited by W.D. van Driel and X.J. Fan, 1-11. New York, NY: Springer New York. https://doi.org/10.1007/978-1-4614-3067-4_1.

Guida, Alessandro, Ahmed M. Megreya, Magali Lavielle-Guida, Yvonnick Noël, Fabien Mathy, Jean-Philippe van Dijck, and Elger Abrahamse. 2018. 'Spatialization in Working Memory Is Related to Literacy and Reading Direction: Culture "Literarily" Directs Our Thoughts'. Cognition 175 (June): 96-100. https://doi.org/10.1016/j.cognition.2018.02.013.

Guo, Jing, Andrew Bakshi, Ying Wang, Longda Jiang, Loic Yengo, Michael E Goddard, Peter M Visscher, and Jian Yang. 2021. 'Quantifying Genetic Heterogeneity between Continental Populations for Human Height and Body Mass Index'. Scientific Reports 11: 5240. https://doi.org/10.1038/s41598-021-84739-z.

Gurdasani, Deepti, Tommy Carstensen, Segun Fatumo, Guanjie Chen, Chris S. Franklin, Javier Prado-Martinez, Heleen Bouman, et al. 2019. 'Uganda Genome Resource Enables Insights into Population History and Genomic Discovery in Africa'. Cell 179 (4): 9841002.e36. https://doi.org/10/ddmk.

Gurven, Michael, Christopher von Rueden, Maxim Massenkoff, Hillard Kaplan, and Marino Lero Vie. 2013. 'How Universal Is the Big Five? Testing the Five-Factor Model of Personality Variation Among Forager-Farmers in the Bolivian Amazon'. Journal of Personality and Social Psychology 104 (2): 354-70. https://doi.org/10/f4nb2z.

Hackman, Daniel A., Martha J. Farah, and Michael J. Meaney. 2010. 'Socioeconomic Status and the Brain: Mechanistic Insights from Human and Animal Research'. Nature Reviews Neuroscience 11 (9): 651-59. https://doi.org/10.1038/nrn2897.

Hamer, D, and L Sirota. 2000. 'Beware the Chopsticks Gene'. Molecular Psychiatry 5: 11-13. https://doi.org/10/ffqv52.

Handley, Carla, and Sarah Mathew. 2020. 'Human Large-Scale Cooperation as a Product of Competition between Cultural Groups'. Nature Communications 11 (1): 702. https://doi.org/10.1038/s41467-020-14416-8.

Hanscombe, Ken B., Maciej Trzaskowski, Claire M. A. Haworth, Oliver S. P. Davis, Philip S. Dale, and Robert Plomin. 2012. 'Socioeconomic Status (SES) and Children’s Intelligence 
(IQ): In a UK-Representative Sample SES Moderates the Environmental, Not Genetic, Effect on IQ'. PLOS ONE 7 (2): e30320. https://doi.org/10/fxnnhk.

Harden, K. Paige. 2021. “Reports of My Death Were Greatly Exaggerated”: Behavior Genetics in the Postgenomic Era'. Annual Review of Psychology 72 (1): annurev-psych-052220103822. https://doi.org/10.1146/annurev-psych-052220-103822.

Harden, K. Paige, and Philipp D. Koellinger. 2020. 'Using Genetics for Social Science'. Nature Human Behaviour 4 (May): 567-76. https://doi.org/10/ggvqb7.

Harris, Judith R. 1995. 'Where Is the Child's Environment? A Group Socialization Theory of Development'. Psychological Review 102 (3): 458-89. https://doi.org/10/cwknnh.

Hatemi, Peter K., Carolyn L. Funk, Sarah E. Medland, Hermine M. Maes, Judy L. Silberg, Nicholas G. Martin, and Lindon J. Eaves. 2009. 'Genetic and Environmental Transmission of Political Attitudes Over a Life Time'. The Journal of Politics 71 (3): 114156. https://doi.org/10.1017/S0022381609090938.

Hatemi, Peter K., Nathan A. Gillespie, Lindon J. Eaves, Brion S. Maher, Bradley T. Webb, Andrew C. Heath, Sarah E. Medland, et al. 2011. 'A Genome-Wide Analysis of Liberal and Conservative Political Attitudes'. The Journal of Politics 73 (1): 271-85. https://doi.org/10.1017/S0022381610001015.

Hawkes, Kristen, and Barbara L. Finlay. 2018. 'Mammalian Brain Development and Our Grandmothering Life History'. Physiology Eं Behavior 193 (September): 55-68. https://doi.org/10.1016/j.physbeh.2018.01.013.

Haworth, C M A, M J Wright, M Luciano, N G Martin, E J C de Geus, C E M van Beijsterveldt, M Bartels, et al. 2010. 'The Heritability of General Cognitive Ability Increases Linearly from Childhood to Young Adulthood'. Molecular Psychiatry 15 (11): 1112-20. https://doi.org/10.1038/mp.2009.55.

Haworth, Claire M. A., and Oliver S. P. Davis. 2014. 'From Observational to Dynamic Genetics'. Frontiers in Genetics 5 (6). https://doi.org/10.3389/fgene.2014.00006.

Healy, J D. 2003. 'Excess Winter Mortality in Europe: A Cross Country Analysis Identifying Key Risk Factors'. Journal of Epidemiology $\mathcal{F}^{\circ}$ Community Health 57 (10): 784-89. https://doi.org/10.1136/jech.57.10.784. 
Heath, Andrew C, Kare Berg, Lindon J Eaves, Marit H Solaas, Linda A Corey, Jon Sundet, Per Magnus, and Walter E Nance. 1985. 'Education Policy and the Heritability of Educational Attainment'. Nature 314 (6013): 734-36.

Heine, Steven J. 2017. DNA Is Not Destiny: The Remarkable, Completely Misunderstood Relationship between You and Your Genes. New York: Norton.

Hellwege, Jacklyn N, Jacob M Keaton, Ayush Giri, Xiaoyi Gao, Digna R Velez Edwards, and Todd L Edwards. 2017. 'Population Stratification in Genetic Association Studies'. Current Protocols in Human Genetics 95: 1.22.1-1.22.23. https://doi.org/10.1002/cphg.48.

Henrich, Joseph. 2001. 'Cultural Transmission and the Diffusion of Innovations: Adoption Dynamics Indicate That Biased Cultural Transmission Is the Predominate Force in Behavioral Change'. American Anthropologist 103 (4): 992-1013. https://doi.org/10.1525/aa.2001.103.4.992.

- 2004a. 'Demography and Cultural Evolution: How Adaptive Cultural Processes Can Produce Maladaptive Losses: The Tasmanian Case'. American Antiquity 69 (2): 197-214.

—. 2004b. 'Cultural Group Selection, Coevolutionary Processes and Large-Scale Cooperation'. Journal of Economic Behavior \& Organization 53 (1): 3-35. https://doi.org/10.1016/S0167-2681(03)00094-5.

- 2016. The Secret of Our Success: How Culture Is Driving Human Evolution, Domesticating Our Species, and Making Us Smarter. Princeton, NJ: Princeton University Press.

- 2020. WEIRDest People in the World: How the West Became Psychologically Peculiar and Particularly Prosperous. London: Allen Lane.

Henrich, Joseph, and James Broesch. 2011. 'On the Nature of Cultural Transmission Networks: Evidence from Fijian Villages for Adaptive Learning Biases'. Philosophical Transactions of the Royal Society B: Biological Sciences 366 (1567): 1139-48. https://doi.org/10/crbg83.

Henrich, Joseph, and Francisco J Gil-White. 2001. 'The Evolution of Prestige: Freely Conferred Deference as a Mechanism for Enhancing the Benefits of Cultural Transmission'. Evolution and Human Behavior 22 (3): 165-96. https://doi.org/10.1016/S10905138(00)00071-4. 
Henrich, Joseph, Steven J. Heine, and Ara Norenzayan. 2010. 'The Weirdest People in the World?' Behavioral and Brain Sciences 33 (2-3): 61-83. https://doi.org/10.1017/S0140525X0999152X.

Henrich, Joseph, and Natalie Henrich. 2010. 'The Evolution of Cultural Adaptations: Fijian Food Taboos Protect against Dangerous Marine Toxins'. Proceedings of the Royal Society B: Biological Sciences 277 (1701): 3715-24. https://doi.org/10.1098/rspb.2010.1191.

Henrich, Joseph, and Michael Muthukrishna. 2021. 'The Origins and Psychology of Human Cooperation'. Annual Review of Psychology 72 (1): 207-40. https://doi.org/10.1146/annurev-psych-081920-042106.

Hewlett, Barry S., Hillary N. Fouts, Adam H. Boyette, and Bonnie L. Hewlett. 2011. 'Social Learning among Congo Basin Hunter-Gatherers'. Philosophical Transactions of the Royal Society B: Biological Sciences 366 (1567): 1168-78. https://doi.org/10.1098/rstb.2010.0373.

Hill, Kim, and A. Magdalena Hurtado. 2009. 'Cooperative Breeding in South American HunterGatherers.' Proceedings of the Royal Society of London. Series B: Biological Sciences. 276 (1674): 3863-70. https://doi.org/10/djbpvr.

Hill, W. David, Saskia P. Hagenaars, Riccardo E. Marioni, Sarah E. Harris, David C.M. Liewald, Gail Davies, Aysu Okbay, Andrew M. McIntosh, Catharine R. Gale, and Ian J. Deary. 2016. 'Molecular Genetic Contributions to Social Deprivation and Household Income in $\begin{array}{llllll}\text { UK Biobank'. } \quad \text { Current } & \text { 3083-89. }\end{array}$ https://doi.org/10.1016/j.cub.2016.09.035.

Hofstede, Geert. 2001. Culture's Consequences: Comparing Values, Behaviors, Institutions and Organizations across Nations. Thousand Oaks, California: Sage publications.

Holick, Michael F. 2007. 'Vitamin D Deficiency'. New England Journal of Medicine 357 (3): 26681. https://doi.org/10.1056/NEJMra070553.

Horwitz, Tanya, Katie Lam, Yu Chen, Yan Xia, and Chunyu Liu. 2019. 'A Decade in Psychiatric GWAS Research'. Molecular Psychiatry 24 (3): 378-89. https://doi.org/10.1038/s41380018-0055-z.

Howard, Janet A., and Mhairi A. Gibson. 2017. 'Frequency-Dependent Female Genital Cutting Behaviour Confers Evolutionary Fitness Benefits'. Nature Ecology Ẽ Evolution 1 (3): 0049. https://doi.org/10.1038/s41559-016-0049. 
Hunter, David J. 2005. 'Gene-Environment Interactions in Human Diseases'. Nature Reviews Genetics 6 (4): 287-98. https://doi.org/10.1038/nrg1578.

Hutchins, Edwin. 1995. Cognition in the Wild. MIT Press.

Inglehart, R., C. Haerpfer, A. Moreno, C. Welzel, K. Kizilova, J. Diez-Medrano, M. Lagos, et al. 2014. 'World Values Survey: All Rounds - Country-Pooled Datafile 1981-2014'. Madrid: JD Jystems Institute. http://www.worldvaluessurvey.org/WVSDocumentationWVL.jsp.

Inglehart, Ronald, and Christian Welzel. 2005. Modernization, Cultural Change, and Democracy: The Human Development Sequence. Cambridge University Press. https://doi.org/10.1017/CBO9780511790881.

Jablonka, Eva, and Marion J. Lamb. 2005. Evolution in Four Dimensions. Cambridge, MA: MIT Press.

Jardim-Botelho, Anne, Sophia Raff, Renato de Ávila Rodrigues, Heather J. Hoffman, David Joseph Diemert, Rodrigo Corrêa-Oliveira, Jeffrey Michael Bethony, and Maria Flávia Gazzinelli. 2008. 'Hookworm, Ascaris Lumbricoides Infection and Polyparasitism Associated with Poor Cognitive Performance in Brazilian Schoolchildren'. Tropical Medicine Ev International Health 13 (8): 994-1004. https://doi.org/10.1111/j.13653156.2008.02103.x.

Jensen, Arthur. 1969. 'How Much Can We Boost IQ and Scholastic Achievement'. Harvard Educational Review 39 (1): 1-123. https://doi.org/10.17763/haer.39.1.13u15956627424k7.

Ji, Ting, Jia-Jia Wu, Qiao-Qiao He, Jing-Jing Xu, Ruth Mace, and Yi Tao. 2013. 'Reproductive Competition between Females in the Matrilineal Mosuo of Southwestern China'. Philosophical Transactions of the Royal Society B: Biological Sciences 368 (1631): 20130081. https://doi.org/10/ggzrzj.

Johnson, Jacqueline S, and Elissa L Newport. 1989. 'Critical Period Effects in Second Language Learning: The Influence of Maturational State on the Acquisition of English as a Second Language'. Cognitive Psychology 21 (1): 60-99. https://doi.org/10.1016/00100285(89)90003-0.

Johnson, Steven. 2006. Everything Bad Is Good for You: How Popular Culture Is Making Us Smarter. London: Penguin Books. 
Jones, Charles. 2020. 'The End of Economic Growth? Unintended Consequences of a Declining Population'. w26651. Cambridge, MA: National Bureau of Economic Research. https://doi.org/10.3386/w26651.

Joshi, Peter K, Tonu Esko, Hannele Mattsson, Niina Eklund, Ilaria Gandin, Teresa Nutile, Anne U Jackson, et al. 2015. 'Directional Dominance on Stature and Cognition in Diverse Human Populations.' Nature 523 (7561): 459-62. https://doi.org/10/f3mzhw.

Kăĝitçibaşi, Cigdem. 1984. 'Socialization in Traditional Society: A Challenge to Psychology'. International Journal of Psychology 19 (1-4): 145-57. https://doi.org/10.1080/00207598408247522.

Kan, Kees-Jan, Jelte M. Wicherts, Conor V. Dolan, and Han L. J. van der Maas. 2013. 'On the Nature and Nurture of Intelligence and Specific Cognitive Abilities: The More Heritable, the More Culture Dependent'. Psychological Science 24 (12): 2420-28. https://doi.org/10/f5kqvg.

Karasik, Lana B., Catherine S. Tamis-LeMonda, Karen E. Adolph, and Marc H. Bornstein. 2015. 'Places and Postures: A Cross-Cultural Comparison of Sitting in 5-Month-Olds'. Journal of Cross-Cultural Psychology 46 (8): 1023-38. https://doi.org/10.1177/0022022115593803.

Kempermann, Gerd. 2019. 'Environmental Enrichment, New Neurons and the Neurobiology of Individuality'. Nature Revierws Neuroscience 20 (4): 235-45. https://doi.org/10.1038/s41583-019-0120-x.

Kendal, Rachel L., Neeltje J. Boogert, Luke Rendell, Kevin N. Laland, Mike Webster, and Patricia L. Jones. 2018. 'Social Learning Strategies: Bridge-Building between Fields'. Trends in Cognitive Sciences 22 (7): 651-65. https://doi.org/10.1016/j.tics.2018.04.003.

Kievit, Rogier, Willem Eduard Frankenhuis, Lourens Waldorp, and Denny Borsboom. 2013. 'Simpson's Paradox in Psychological Science: A Practical Guide'. Frontiers in Psychology 4: 513. https://doi.org/10.3389/fpsyg.2013.00513.

Kim, Michelle S., Kane P. Patel, Andrew K. Teng, Ali J. Berens, and Joseph Lachance. 2018. 'Genetic Disease Risks Can Be Misestimated across Global Populations'. Genome Biology 19 (1): 179 . https://doi.org/10/ggsmzz.

Kirkpatrick, Robert M., Matt McGue, and William G. Iacono. 2015. 'Replication of a GeneEnvironment Interaction Via Multimodel Inference: Additive-Genetic Variance in 
Adolescents' General Cognitive Ability Increases with Family-of-Origin Socioeconomic Status'. Behavior Genetics 45 (2): 200-214. https://doi.org/10.1007/s10519-014-9698-y.

Kitayama, Shinobu, Sean Duffy, Tadashi Kawamura, and Jeff T. Larsen. 2003. 'Perceiving an Object and Its Context in Different Cultures: A Cultural Look at New Look'. Psychological Science 14 (3): 201-6. https://doi.org/10/djvkd5.

Kline, Michelle A., Robert Boyd, and Joseph Henrich. 2013. 'Teaching and the Life History of Cultural Transmission in Fijian Villages'. Human Nature 24 (4): 351-74. https://doi.org/10.1007/s12110-013-9180-1.

Kolodny, Oren, Nicole Creanza, and Marcus W. Feldman. 2015. 'Evolution in Leaps: The Punctuated Accumulation and Loss of Cultural Innovations'. Proceedings of the National Academy of Sciences 112 (49): E6762-69. https://doi.org/10/gfzkmp.

Kremen, William S., Kristen C. Jacobson, Hong Xian, Seth A. Eisen, Brian Waterman, Rosemary Toomey, Michael C. Neale, Ming T. Tsuang, and Michael J. Lyons. 2005. 'Heritability of Word Recognition in Middle-Aged Men Varies as a Function of Parental Education'. Behavior Genetics 35 (4): 417-33. https://doi.org/10.1007/s10519-004-3876-2.

Kuhl, P., K. Williams, F Lacerda, K. Stevens, and B Lindblom. 1992. 'Linguistic Experience Alters Phonetic Perception in Infants by 6 Months of Age'. Science 255 (5044): 606-8. https://doi.org/10.1126/science.1736364.

Laland, K. N., and M. J. O’Brien. 2011. 'Cultural Niche Construction: An Introduction'. Biological Theory laland (3): 191-202. https://doi.org/10.1007/s13752-012-0026-6.

Laland, K. N., J. Odling-Smee, and M. W. Feldman. 2001. 'Cultural Niche Construction and Human Evolution'. Journal of Evolutionary Biology 14 (1): 22-33. https://doi.org/10.1046/j.1420-9101.2001.00262.x.

Laland, Kevin, Blake Matthews, and Marcus W. Feldman. 2016. 'An Introduction to Niche Construction Theory'. Evolutionary Ecology $30 \quad$ (2): 191-202. https://doi.org/10.1007/s10682-016-9821-z.

Laland, Kevin N. 2018. Darwin's Unfinished Symphony: How Culture Made the Human Mind. Princeton, NJ: Princeton University Press. 
Laland, Kevin N., John Odling-Smee, and Sean Myles. 2010. 'How Culture Shaped the Human Genome: Bringing Genetics and the Human Sciences Together.' Nature Reviews Genetics 11 (2): 137-48. https://doi.org/10/cr7cmf.

Laland, Kevin N., Tobias Uller, Marcus W. Feldman, Kim Sterelny, Gerd B. Müller, Armin Moczek, Eva Jablonka, and John Odling-Smee. 2015. 'The Extended Evolutionary Synthesis: Its Structure, Assumptions and Predictions'. Proceedings of the Royal Society B: Biological Sciences 282 (1813): 20151019. https://doi.org/10/f3n9qx.

Lamb, Jonathan, James May, and Fiona Harrison. 2017. Scurvy: The Disease of Discovery. Princeton University Press. https://doi.org/10.2307/j.ctt21c4vc3.

Leary, Patrick F., Ina Zamfirova, Johnathan Au, and Ward H. McCracken. 2017. 'Effect of Latitude on Vitamin D Levels'. The Journal of the American Osteopathic Association 117 (7): 433. https://doi.org/10.7556/jaoa.2017.089.

Lee, James J., Robbee Wedow, Aysu Okbay, Edward Kong, Omeed Maghzian, Meghan Zacher, Tuan Anh Nguyen-Viet, et al. 2018. 'Gene Discovery and Polygenic Prediction from a Genome-Wide Association Study of Educational Attainment in 1.1 Million Individuals'. Nature Genetics 50 (8): 1112-21. https://doi.org/10/gdvmq2.

Legare, Cristine H., and Mark Nielsen. 2015. 'Imitation and Innovation: The Dual Engines of Cultural Learning'. Trends in Cognitive Sciences 19 (11): 688-99. https://doi.org/10/f7zb9w.

Lek, Monkol, Konrad J. Karczewski, Eric V. Minikel, Kaitlin E. Samocha, Eric Banks, Timothy Fennell, Anne H. O'Donnell-Luria, et al. 2016. 'Analysis of Protein-Coding Genetic Variation in 60,706 Humans'. Nature 536 (7616): 285-91. https://doi.org/10.1038/nature19057.

Lewontin, Richard C. 1970. 'Race and Intelligence'. Bulletin of the Atomic Scientists 26 (3): 2-8. https://doi.org/10.1080/00963402.1970.11457774.

- 1974. 'Annotation: The Analysis of Variance and the Analysis of Causes.' American Journal of Human Genetics 26: 400-411.

Lieblich, Amia. 2010. 'A Century of Childhood, Parenting, and Family Life in the Kibbutz'. Journal of Israeli History 29 (1): 1-24. https://doi.org/10.1080/13531041003594608. 
Lips, Paul. 2001. 'Vitamin D Deficiency and Secondary Hyperparathyroidism in the Elderly: Consequences for Bone Loss and Fractures and Therapeutic Implications'. Endocrine Reviews 22 (4): 477-501.

- 2007. 'Vitamin D Status and Nutrition in Europe and Asia'. The Journal of Steroid Biochemistry and Molecular Biology 103 (3-5): 620-25. https://doi.org/10.1016/j.jsbmb.2006.12.076.

Lips, Paul, Tu Duong, Anna Oleksik, Dennis Black, Steven Cummings, David Cox, and Thomas Nickelsen. 2001. 'A Global Study of Vitamin D Status and Parathyroid Function in Postmenopausal Women with Osteoporosis: Baseline Data from the Multiple Outcomes of Raloxifene Evaluation Clinical Trial'. The Journal of Clinical Endocrinology \& Metabolism 86 (3): 10.

Lipschuetz, Michal, Sarah M. Cohen, Eliana Ein-Mor, Hanna Sapir, Drorith Hochner-Celnikier, Shay Porat, Hagai Amsalem, et al. 2015. 'A Large Head Circumference Is More Strongly Associated with Unplanned Cesarean or Instrumental Delivery and Neonatal Complications than High Birthweight'. American Journal of Obstetrics and Gynecology 213 (6): 833.e1-833.e12. https://doi.org/10/f72gz4.

Lupyan, Gary, Rasha Abdel Rahman, Lera Boroditsky, and Andy Clark. 2020. 'Effects of Language on Visual Perception'. Trends in Cognitive Sciences 24 (11): 930-44. https://doi.org/10.1016/j.tics.2020.08.005.

Lynn, Richard. 1990. 'The Role of Nutrition in Secular Increases in Intelligence'. Personality and Individual Differences 11 (3): 273-85. https://doi.org/10.1016/0191-8869(90)90241-I.

Lynn, Richard, and Tatu Vanhanen. 2012. Intelligence: A Unifying Construct for the Social Sciences. London: Ulster Institute for Social Research.

Majid, Asifa, Melissa Bowerman, Sotaro Kita, Daniel B.M. Haun, and Stephen C. Levinson. 2004. 'Can Language Restructure Cognition? The Case for Space'. Trends in Cognitive Sciences 8 (3): 108-14. https://doi.org/10.1016/j.tics.2004.01.003.

Mallick, Swapan, Heng Li, Mark Lipson, Iain Mathieson, Melissa Gymrek, Fernando Racimo, Mengyao Zhao, et al. 2016. 'The Simons Genome Diversity Project: 300 Genomes from 142 Diverse Populations'. Nature 538 (7624): 201-6. https://doi.org/10.1038/nature18964. 
Martin, Alicia R., Christopher R. Gignoux, Raymond K. Walters, Genevieve L. Wojcik, Benjamin M. Neale, Simon Gravel, Mark J. Daly, Carlos D. Bustamante, and Eimear E. Kenny. 2017. 'Human Demographic History Impacts Genetic Risk Prediction across Diverse Populations'. The American Journal of Human Genetics 100 (4): 635-49. https://doi.org/10/f92trq.

Martin, Alicia R., Masahiro Kanai, Yoichiro Kamatani, Yukinori Okada, Benjamin M. Neale, and Mark J. Daly. 2019. 'Clinical Use of Current Polygenic Risk Scores May Exacerbate Health Disparities'. Nature Genetics 51 (4): 584-91. https://doi.org/10.1038/s41588-0190379-x.

Martin, Gregory J., and Ali Yurukoglu. 2017. 'Bias in Cable News: Persuasion and Polarization'. American Economic Review 107 (9): 2565-99. https://doi.org/10/gbzjkj.

McKee, CM. 1989. 'Deaths in Winter: Can Britain Learn from Europe?' European Journal of Epidemiology 5 (2): 178-82.

Medin, Douglas L., and Scott Atran. 2004. 'The Native Mind: Biological Categorization and Reasoning in Development and Across Cultures.' Psychological Review 111 (4): 960-83. https://doi.org/10.1037/0033-295X.111.4.960.

Meltzer, David O., Thomas J. Best, Hui Zhang, Tamara Vokes, Vineet Arora, and Julian Solway. 2020. 'Association of Vitamin D Status and Other Clinical Characteristics With COVID19 Test Results'. JAMA Network Open 3 (9): $\quad$ e2019722. https://doi.org/10.1001/jamanetworkopen.2020.19722.

Mesoudi, Alex. 2011. 'Variable Cultural Acquisition Costs Constrain Cumulative Cultural Evolution'. Edited by R. Bentley. PLoS ONE 6 (3): e18239. https://doi.org/10.1371/journal.pone.0018239.

Michalopoulos, Stelios, and Elias Papaioannou. 2020. 'Historical Legacies and African Development'. Journal of Economic Literature 58 (1): 53-128. https://doi.org/10.1257/jel.20181447.

Mills, Melinda C., and Charles Rahal. 2019. 'A Scientometric Review of Genome-Wide Association Studies'. Communications Biology 2 (1). https://doi.org/10.1038/s42003-0180261-x. 
Mills, Melinda C., and Felix C. Tropf. 2020. 'Sociology, Genetics, and the Coming of Age of Sociogenomics'. Annual Review of Sociology 46 (1): 553-81. https://doi.org/10/ggvqs7.

Mithal, A., D. A. Wahl, J.-P. Bonjour, P. Burckhardt, B. Dawson-Hughes, J. A. Eisman, G. E1Hajj Fuleihan, R. G. Josse, P. Lips, and J. Morales-Torres. 2009. 'Global Vitamin D Status and Determinants of Hypovitaminosis D'. Osteoporosis International 20 (11): 1807-20. https://doi.org/10.1007/s00198-009-0954-6.

Moffitt, Terrie E., Avshalom Caspi, and Michael Rutter. 2005. 'Strategy for Investigating Interactions Between Measured Genes and Measured Environments'. Archives of General Psychiatry 62 (5): 473. https://doi.org/10.1001/archpsyc.62.5.473.

Montague, Meg, Ron Borland, and Craig Sinclair. 2001. 'Slip! Slop! Slap! And SunSmart, 19802000: Skin Cancer Control and 20 Years of Population-Based Campaigning'. Health Education E Behavior 28 (3): 290-305. https://doi.org/10/chvbts.

Moore, David S., and David Shenk. 2016. 'The Heritability Fallacy'. Wiley Interdisciplinary Reviews: Cognitive Science 8 (1-2): e1400. https://doi.org/10.1002/wcs.1400.

Morgan, Thomas J.H., Jordan W. Suchow, and Thomas L. Griffiths. 2020. 'What the Baldwin Effect Affects Depends on the Nature of Plasticity'. Cognition 197 (April): 104165. https://doi.org/10.1016/j.cognition.2019.104165.

Morgan, Thomas JH, Natalie T Uomini, Luke E Rendell, Laura Chouinard-Thuly, Sharon E Street, Hannah M Lewis, Catherine P Cross, et al. 2015. 'Experimental Evidence for the Co-Evolution of Hominin Tool-Making Teaching and Language'. Nature Communications 6 (1): 1-8.

Morris, Tim T., Neil M. Davies, Gibran Hemani, and George Davey Smith. 2020. 'Population Phenomena Inflate Genetic Associations of Complex Social Traits'. Science Advances 6 (16): eaay0328. https://doi.org/10/ggxrcn.

Mostafavi, Hakhamanesh, Arbel Harpak, Ipsita Agarwal, Dalton Conley, Jonathan K. Pritchard, and Molly Przeworski. 2020. 'Variable Prediction Accuracy of Polygenic Scores within an Ancestry Group'. ELife 9 (January): e48376. https://doi.org/10/ggrfbs.

Murray, Charles. 2020. Human Diversity: The Biology of Gender, Race, and Class. New York: Twelve. Muthukrishna, Michael, Adrian V Bell, Joseph Henrich, Cameron M Curtin, Alexander Gedranovich, Jason McInerney, and Braden Thue. 2020. 'Beyond Western, Educated, 
Industrial, Rich, and Democratic (WEIRD) Psychology: Measuring and Mapping Scales of Cultural and Psychological Distance'. Psychological Science 31 (6): 678-701. https://doi.org/10/ggxkjb.

Muthukrishna, Michael, Michael Doebeli, Maciej Chudek, and Joseph Henrich. 2018. 'The Cultural Brain Hypothesis: How Culture Drives Brain Expansion, Sociality, and Life History'. Edited by Corina E Tarnita. PLOS Computational Biology 14 (11): e1006504. https://doi.org/10.1371/journal.pcbi.1006504.

Muthukrishna, Michael, and Joseph Henrich. 2016. 'Innovation in the Collective Brain'. Philosophical Transactions of the Royal Society B: Biological Sciences 371 (1690): 20150192. https://doi.org/10.1098/rstb.2015.0192.

—. 2019. 'A Problem in Theory'. Nature Human Behaviour 3 (March): 221-29. https://doi.org/10/gfvdx8.

Muthukrishna, Michael, Joseph Henrich, and Edward Slingerland. 2021. 'Psychology as a Historical Science'. Annual Review of Psychology 72 (1): 717-49. https://doi.org/10/ghrnb6. Muthukrishna, Michael, Thomas J.H. Morgan, and Joseph Henrich. 2016. 'The When and Who of Social Learning and Conformist Transmission'. Evolution and Human Behavior 37 (1): 10-20. https://doi.org/10/f77jjx.

Muthukrishna, Michael, and Mark Schaller. 2020. 'Are Collectivistic Cultures More Prone to Rapid Transformation? Computational Models of Cross-Cultural Differences, Social Network Structure, Dynamic Social Influence, and Cultural Change'. Personality and Social Psychology Review 24 (2): 103-20. https://doi.org/10.1177/1088868319855783.

Muthukrishna, Michael, Ben W Shulman, Vlad Vasilescu, and Joseph Henrich. 2014. 'Sociality Influences Cultural Complexity'. Proceedings of the Royal Society B: Biological Sciences 281 (1774): 20132511.

Need, Anna C., and David B. Goldstein. 2009. 'Next Generation Disparities in Human Genomics: Concerns and Remedies'. Trends in Genetics 25 (11): 489-94. https://doi.org/10.1016/j.tig.2009.09.012.

Needleman, Herbert L, and Constantine A Gatsonis. 1990. 'Low-Level Lead Exposure and the IQ of Children'. JAMA $263 \quad$ (5): 673-78. https://doi.org/10.1001/jama.1990.03440050067035. 
Nielsen, Mark, Daniel Haun, Joscha Kärtner, and Cristine H. Legare. 2017. 'The Persistent Sampling Bias in Developmental Psychology: A Call to Action'. Journal of Experimental Child Psychology 162 (October): 31-38. https://doi.org/10/gf8x27.

Nisbett, Richard. 2003. The Geography of Thought: How Asians and Westerners Think Differently... and Why. New York: Simon and Schuster.

Nisbett, Richard E. 2009. Intelligence and How to Get It: Why Schools and Cultures Count. 1st ed. New York: W.W. Norton \& Co.

Nisbett, Richard E., Joshua Aronson, Clancy Blair, William Dickens, James Flynn, Diane F. Halpern, and Eric Turkheimer. 2012. 'Intelligence: New Findings and Theoretical Developments.' American Psychologist 67 (2): 130-59. https://doi.org/10.1037/a0026699.

Nunn, Nathan. 2020. 'The Historical Roots of Economic Development'. Science 367 (6485): eaaz9986. https://doi.org/10/ggr7bj.

Olalde, Iñigo, Selina Brace, Morten E. Allentoft, Ian Armit, Kristian Kristiansen, Thomas Booth, Nadin Rohland, et al. 2018. 'The Beaker Phenomenon and the Genomic Transformation of Northwest Europe'. Nature 555 (7695): 190-96. https://doi.org/10.1038/nature25738.

Onalaja, Ava O., and Luz Claudio. 2000. 'Genetic Susceptibility to Lead Poisoning'. Environmental Health Perspectives 108 (suppl 1): 23-28. https://doi.org/10.1289/ehp.00108s123.

Ong, Walter J. 1982. Orality and Literacy: The Technologizing of the Word. London: Methuen.

Osada, Naoki, and Hiroshi Akashi. 2012. 'Mitochondrial-Nuclear Interactions and Accelerated Compensatory Evolution: Evidence from the Primate Cytochrome c Oxidase Complex'. Molecular Biology and Evolution 29 (1): 337-46. https://doi.org/10.1093/molbev/msr211.

Oskina, N. A., N. A. Ermolenko, U. A. Boyarskih, A. F. Lazarev, V. D. Petrova, D. I. Ganov, O. G. Tonacheva, G. I. Lifschitz, and M. L. Filipenko. 2014. 'Associations Between SNPs Within Antioxidant Genes and the Risk of Prostate Cancer in the Siberian Region of Russia'. Pathology E Oncology Research 20 (3): 635-40. https://doi.org/10.1007/s12253014-9742-5.

Pagani, Luca, Toomas Kivisild, Ayele Tarekegn, Rosemary Ekong, Chris Plaster, Irene Gallego Romero, Qasim Ayub, et al. 2012. 'Ethiopian Genetic Diversity Reveals Linguistic Stratification and Complex Influences on the Ethiopian Gene Pool'. The 
American Journal of Human Genetics 91 (1): 83-96. https://doi.org/10.1016/j.ajhg.2012.05.015.

Papassotiropoulos, A, K Henke, E Stefanova, A Aerni, A Müller, P Demougin, C Vogler, et al. 2011. 'A Genome-Wide Survey of Human Short-Term Memory'. Molecular Psychiatry 16 (2): 184-92. https://doi.org/10.1038/mp.2009.133.

Pemberton, Trevor J., Paul Verdu, Noémie S. Becker, Cristen J. Willer, Barry S. Hewlett, Sylvie Le Bomin, Alain Froment, Noah A. Rosenberg, and Evelyne Heyer. 2018. 'A Genome Scan for Genes Underlying Adult Body Size Differences between Central African HunterGatherers and Farmers'. Human Genetics 137 (6-7): 487-509. https://doi.org/10/gdzjtx.

Penn, Derek C., and Daniel J. Povinelli. 2007. 'Causal Cognition in Human and Nonhuman Animals: A Comparative, Critical Review'. Annual Review of Psychology 58 (1): 97-118. https://doi.org/10.1146/annurev.psych.58.110405.085555.

Peter, Benjamin M, Desislava Petkova, and John Novembre. 2020. 'Genetic Landscapes Reveal How Human Genetic Diversity Aligns with Geography'. Edited by Evelyne Heyer. Molecular Biology and Evolution 37 (4): 943-51. https://doi.org/10.1093/molbev/msz280.

Petkova, Desislava, John Novembre, and Matthew Stephens. 2016. 'Visualizing Spatial Population Structure with Estimated Effective Migration Surfaces'. Nature Genetics 48 (1): 94-100. https://doi.org/10.1038/ng.3464.

Phillips, Patrick C. 2008. 'Epistasis - the Essential Role of Gene Interactions in the Structure and Evolution of Genetic Systems'. Nature Reviews Genetics 9 (11): 855-67. https://doi.org/10.1038/nrg2452.

Pietschnig, J., and M. Voracek. 2015. 'One Century of Global IQ Gains: A Formal Meta-Analysis of the Flynn Effect (1909-2013)'. Perspectives on Psychological Science 10 (3): 282-306. https://doi.org/10/f7dg4q.

Pilz, Stefan, Winfried März, Kevin D. Cashman, Mairead E. Kiely, Susan J. Whiting, Michael F. Holick, William B. Grant, et al. 2018. 'Rationale and Plan for Vitamin D Food Fortification: A Review and Guidance Paper'. Frontiers in Endocrinology 9 (July): 373. https://doi.org/10.3389/fendo.2018.00373.

Platt, Jonathan M., Katherine M. Keyes, Katie A. McLaughlin, and Alan S. Kaufman. 2019. ‘The Flynn Effect for Fluid IQ May Not Generalize to All Ages or Ability Levels: A 
Population-Based Study of 10,000 US Adolescents'. Intelligence 77 (November): 101385. https://doi.org/10/ggc5kh.

Plomin, Robert. 2019. Blueprint: How DNA Makes Us Who We Are. Cambridge, MA: MIT Press. Plomin, Robert, Kathryn Asbury, and Judith Dunn. 2001. 'Why Are Children in the Same Family So Different? Nonshared Environment a Decade Later'. The Canadian Journal of Psychiatry 46 (3): 225-33. https://doi.org/10.1177/070674370104600302.

Plomin, Robert, and Denise Daniels. 1987. 'Children in the Same Family Are Very Different, but Why?' Behavioral and Brain Sciences $10 \quad$ (1): 44-59. https://doi.org/10.1017/S0140525X00056272.

Plomin, Robert, J. C. DeFries, and John C. Loehlin. 1977. 'Genotype-Environment Interaction and Correlation in the Analysis of Human Behavior'. Psychological Bulletin 84 (2): 309-22. https://doi.org/10.1037/0033-2909.84.2.309.

Plomin, Robert, John C. DeFries, Valerie S. Knopik, and Jenae M. Neiderhiser. 2016. 'Top 10 Replicated Findings From Behavioral Genetics'. Perspectives on Psychological Science 11 (1): 3-23. https://doi.org/10.1177/1745691615617439.

Plomin, Robert, and Sophie von Stumm. 2018. 'The New Genetics of Intelligence'. Nature Reviews Genetics 19: 148-59. https://doi.org/10/gctn8m.

Polderman, Tinca J C, Beben Benyamin, Christiaan A de Leeuw, Patrick F Sullivan, Arjen van Bochoven, Peter M Visscher, and Danielle Posthuma. 2015. 'Meta-Analysis of the Heritability of Human Traits Based on Fifty Years of Twin Studies'. Nature Genetics 47 (7): 702-9. https://doi.org/10/f3nbfg.

Pollet, Thomas V., and Tamsin K. Saxton. 2019. 'How Diverse Are the Samples Used in the Journals "Evolution \& Human Behavior" and "Evolutionary Psychology"? Evolutionary Psychological Science 5 (3): 357-68. https://doi.org/10/ggfptr.

Popejoy, Alice B., and Stephanie M. Fullerton. 2016. 'Genomics Is Failing on Diversity'. Nature 538 (7624): 161-64. https://doi.org/10.1038/538161a.

Prado-Martinez, Javier, Peter H. Sudmant, Jeffrey M. Kidd, Heng Li, Joanna L. Kelley, Belen Lorente-Galdos, Krishna R. Veeramah, et al. 2013. 'Great Ape Genetic Diversity and Population History'. Nature 499 (7459): 471-75. https://doi.org/10.1038/nature12228. 
Price, Alkes L., Noah A. Zaitlen, David Reich, and Nick Patterson. 2010. 'New Approaches to Population Stratification in Genome-Wide Association Studies'. Nature Revierws Genetics 11 (7): 459-63. https://doi.org/10.1038/nrg2813.

Purcell, Shaun. 2002. 'Variance Components Models for Gene-Environment Interaction in Twin Analysis'. Twin Research 5 (6): 554-71. https://doi.org/10/fsmt2b.

Ravallion, Martin. 2016. The Economics of Poverty: History, Measurement, and Policy. Oxford University Press. https://doi.org/10.1093/acprof:oso/9780190212766.001.0001.

Rhodes, J. M., S. Subramanian, E. Laird, G. Griffin, and R. A. Kenny. 2020. 'Perspective: Vitamin D Deficiency and COVID-19 Severity - Plausibly Linked by Latitude, Ethnicity, Impacts on Cytokines, ACE2 and Thrombosis'. Journal of Internal Medicine, July, joim.13149. https://doi.org/10.1111/joim.13149.

Richerson, P. J., R. Boyd, and J. Henrich. 2010. 'Gene-Culture Coevolution in the Age of Genomics'. Proceedings of the National Academy of Sciences 107 (Supplement_2): 8985-92. https://doi.org/10.1073/pnas.0914631107.

Richerson, Peter, Ryan Baldini, Adrian V. Bell, Kathryn Demps, Karl Frost, Vicken Hillis, Sarah Mathew, et al. 2016. 'Cultural Group Selection Plays an Essential Role in Explaining Human Cooperation: A Sketch of the Evidence'. Behavioral and Brain Sciences 39 (May): e30. https://doi.org/10.1017/S0140525X1400106X.

Rimfeld, Kaili, Eva Krapohl, Maciej Trzaskowski, Jonathan R. I. Coleman, Saskia Selzam, Philip S. Dale, Tonu Esko, Andres Metspalu, and Robert Plomin. 2018. 'Genetic Influence on Social Outcomes during and after the Soviet Era in Estonia'. Nature Human Bebaviour 2 (4): 269-75. https://doi.org/10.1038/s41562-018-0332-5.

Ritchie, Stuart J, and Elliot M Tucker-Drob. 2018. 'How Much Does Education Improve Intelligence? A Meta-Analysis'. Psychological Science 29 (8): 1358-69. https://doi.org/10.1177/0956797618774253.

Rogers, Alan R. 1988. 'Does Biology Constrain Culture?' American Anthropologist 90 (4): 819-31. https://doi.org/10.1525/aa.1988.90.4.02a00030.

Rogers, Everett M. 2003. Diffusion of Innovations. New York: Free Press.

Rosenberg, Noah A., Michael D. Edge, Jonathan K. Pritchard, and Marcus W. Feldman. 2019. 'Interpreting Polygenic Scores, Polygenic Adaptation, and Human Phenotypic 
Differences'. Evolution, Medicine, and Public Health 2018 (1): 26-34. https://doi.org/10/gftgwv.

Rosenzweig, Mark R., and Edward L. Bennett. 1996. 'Psychobiology of Plasticity: Effects of Training and Experience on Brain and Behavior'. Behavioural Brain Research 78 (1): 5765. https://doi.org/10.1016/0166-4328(95)00216-2.

Rotimi, Charles N., Amy R. Bentley, Ayo P. Doumatey, Guanjie Chen, Daniel Shriner, and Adebowale Adeyemo. 2017. 'The Genomic Landscape of African Populations in Health and Disease'. Human Molecular Genetics 26 (R2): R225-36. https://doi.org/10/gbzx3g.

Rowe, David C., Kristen C. Jacobson, and Edwin J. C. G. Van den Oord. 1999. 'Genetic and Environmental Influences on Vocabulary IQ: Parental Education Level as Moderator'. Child Development 70 (5): 1151-62. https://doi.org/10.1111/1467-8624.00084.

Rutter, Michael. 2007. 'Gene-Environment Interdependence'. Developmental Science 10 (1): 1218. https://doi.org/10.1111/j.1467-7687.2007.00557.x.

Samuelsson, Stefan, Brian Byrne, Richard K. Olson, Jacqueline Hulslander, Sally Wadsworth, Robin Corley, Erik G. Willcutt, and John C. DeFries. 2008. 'Response to Early Literacy Instruction in the United States, Australia, and Scandinavia: A Behavioral-Genetic Analysis'. Learning and Individual Differences 18 (3): 289-95. https://doi.org/10.1016/j.lindif.2008.03.004.

Sanchez-Roige, Sandra, Pierre Fontanillas, Sarah L. Elson, the 23andMe Research Team, Anita Pandit, Ellen M. Schmidt, Johanna R. Foerster, et al. 2018. 'Genome-Wide Association Study of Delay Discounting in 23,217 Adult Research Participants of European Ancestry'. Nature Neuroscience 21 (1): 16-18. https://doi.org/10.1038/s41593-017-0032-x.

Sanjak, Jaleal S., Julia Sidorenko, Matthew R. Robinson, Kevin R. Thornton, and Peter M. Visscher. 2018. 'Evidence of Directional and Stabilizing Selection in Contemporary Humans'. Proceedings of the National Academy of Sciences 115 (1): 151-56. https://doi.org/10.1073/pnas.1707227114.

Sauce, Bruno, Sophie Bendrath, Margalit Herzfeld, Dan Siegel, Conner Style, Sayeeda Rab, Jonathan Korabelnikov, and Louis D. Matzel. 2018. 'The Impact of Environmental Interventions among Mouse Siblings on the Heritability and Malleability of General 
Cognitive Ability'. Philosophical Transactions of the Royal Society B: Biological Sciences 373 (1756): 20170289. https://doi.org/10.1098/rstb.2017.0289.

Savage, Jeanne E., Philip R. Jansen, Sven Stringer, Kyoko Watanabe, Julien Bryois, Christiaan A. de Leeuw, Mats Nagel, et al. 2018. 'Genome-Wide Association Meta-Analysis in 269,867 Individuals Identifies New Genetic and Functional Links to Intelligence'. Nature Genetics 50 (7): 912-19. https://doi.org/10/gdqb9d.

Scarr, Sandra. 1992. 'Developmental Theories for the 1990s: Development and Individual Differences'. Child Development 63 (1): 1-19. https://doi.org/10.2307/1130897.

Scarr-Salapatek, Sandra. 1971. 'Race, Social Class, and IQ'. Science 174 (4016): 1285-95. https://doi.org/10.1126/science.174.4016.1285.

Schaller, Mark, and Michael Muthukrishna. in press. 'Modeling Cultural Change: Computational Models of Interpersonal Influence Dynamics Can Yield New Insights about How Cultures Change, Which Cultures Change More Rapidly Than Others, And Why'. American Psychologist.

Schirwani, Schaida, Emma Wakeling, Kath Smith, DDD Study, and Meena Balasubramanian. 2018. 'Expanding the Molecular Basis and Phenotypic Spectrum of ZDHHC9 -Associated X-Linked Intellectual Disability'. American Journal of Medical Genetics Part A 176 (5): 1238-44. https://doi.org/10/gdfcfx.

Schulz, Jonathan F., Duman Bahrami-Rad, Jonathan P. Beauchamp, and Joseph Henrich. 2019. 'The Church, Intensive Kinship, and Global Psychological Variation'. Science 366 (6466): eaau5141. https://doi.org/10.1126/science.aau5141.

Schwartz, Christine R. 2013. 'Trends and Variation in Assortative Mating: Causes and Consequences'. Annual Review of Sociology 39 (1): 451-70. https://doi.org/10.1146/annurev-soc-071312-145544.

Selita, Fatos, and Yulia Kovas. 2019. 'Genes and Gini: What Inequality Means for Heritability'. Journal of Biosocial Science 51 (1): 18-47. https://doi.org/10/gf8tkz.

Shemesh, Yair, Yehezkel Sztainberg, Oren Forkosh, Tamar Shlapobersky, Alon Chen, and Elad Schneidman. 2013. 'High-Order Social Interactions in Groups of Mice'. ELife 2: e00759. https://doi.org/10.7554/eLife.00759.001. 
Silventoinen, Karri, Aline Jelenkovic, Reijo Sund, Antti Latvala, Chika Honda, Fujio Inui, Rie Tomizawa, et al. 2020. 'Genetic and Environmental Variation in Educational Attainment: An Individual-Based Analysis of 28 Twin Cohorts'. Scientific Reports 10 (1): 12681. https://doi.org/10.1038/s41598-020-69526-6.

Simpson, E. H. 1951. 'The Interpretation of Interaction in Contingency Tables'. Journal of the Royal Statistical Society: Series B $\quad$ (Methodological) 13 (2): 238-41. https://doi.org/10.1111/j.2517-6161.1951.tb00088.x.

Sirugo, Giorgio, Scott M. Williams, and Sarah A. Tishkoff. 2019. 'The Missing Diversity in Human Genetic Studies'. Cell 177 (1): 26-31. https://doi.org/10/gfxcjg.

Sisk, Victoria F., Alexander P. Burgoyne, Jingze Sun, Jennifer L. Butler, and Brooke N. Macnamara. 2018. 'To What Extent and Under Which Circumstances Are Growth MindSets Important to Academic Achievement? Two Meta-Analyses'. Psychological Science 29 (4): 549-71. https://doi.org/10/gdfhb5.

Sloan, Daniel B., Jessica M. Warren, Alissa M. Williams, Zhiqiang Wu, Salah E. Abdel-Ghany, Adam J. Chicco, and Justin C. Havird. 2018. 'Cytonuclear Integration and Co-Evolution'. Nature Reviews Genetics 19 (10): 635-48. https://doi.org/10.1038/s41576-018-0035-9.

Sluis, Sophie van der, Gonneke Willemsen, Eco J. C. de Geus, Dorret I. Boomsma, and Danielle Posthuma. 2008. 'Gene-Environment Interaction in Adults' IQ Scores: Measures of Past and Present Environment'. Behavior Genetics 38 (4): 348-60. https://doi.org/10/cgmr3d.

Smaldino, Paul E., Aaron Lukaszewski, Christopher von Rueden, and Michael Gurven. 2019. 'Niche Diversity Can Explain Cross-Cultural Differences in Personality Structure'. Nature Human Behaviour 3 (12): 1276-83. https://doi.org/10/gf8db9.

Sniekers, Suzanne, Sven Stringer, Kyoko Watanabe, Philip R. Jansen, Jonathan R.I. Coleman, Eva Krapohl, Erdogan Taskesen, et al. 2017. 'Genome-Wide Association Meta-Analysis of 78,308 Individuals Identifies New Loci and Genes Influencing Human Intelligence'. Nature Genetics 49 (7): 1107-12. https://doi.org/10/b7gm.

Sohail, Mashaal, Robert M Maier, Andrea Ganna, Alex Bloemendal, Alicia R Martin, Michael C Turchin, Charleston WK Chiang, et al. 2019. 'Polygenic Adaptation on Height Is Overestimated Due to Uncorrected Stratification in Genome-Wide Association Studies'. ELife 8 (March): e39702. https://doi.org/10/gf4d2s. 
Soucy, Shannon M., Jinling Huang, and Johann Peter Gogarten. 2015. 'Horizontal Gene Transfer: Building the Web of Life'. Nature Reviews Genetics 16 (8): 472-82. https://doi.org/10.1038/nrg3962.

Spiro, A., and J. L. Buttriss. 2014. 'Vitamin D: An Overview of Vitamin D Status and Intake in Europe: Vitamin D Status and Intake in Europe'. Nutrition Bulletin 39 (4): 322-50. https://doi.org/10.1111/nbu.12108.

Stoch, M. B., P. M. Smythe, A. D. Moodie, and D. Bradshaw. 1982. 'Psychosocial Outcome and CT Findings after Gross Undernourishment during Infancy: A 20-Year Developmental Study'. Developmental Medicine \& Child Neurology 24 (5): 419-36. https://doi.org/10.1111/j.1469-8749.1982.tb13647.x.

Stoolmiller, Mike. 1999. 'Implications of the Restricted Range of Family Environments for Estimates of Heritability and Nonshared Environment in Behavior-Genetic Adoption Studies'. Psychological Bulletin 125 (4): 392-409.

Sturm, Richard A, and David L Duffy. 2012. 'Human Pigmentation Genes under Environmental Selection'. Genome Biology 13 (9): 248. https://doi.org/10.1186/gb-2012-13-9-248.

Swallow, Khena M., and Qi Wang. 2020. 'Culture Influences How People Divide Continuous Sensory Experience into Events'. Cognition 205. https://doi.org/10.1016/j.cognition.2020.104450.

Talhelm, T., X. Zhang, S. Oishi, C. Shimin, D. Duan, X. Lan, and S. Kitayama. 2014. 'LargeScale Psychological Differences Within China Explained by Rice Versus Wheat Agriculture'. Science 344 (6184): 603-8. https://doi.org/10.1126/science.1246850.

Tan, Li Hai, Angela R. Laird, Karl Li, and Peter T. Fox. 2005. 'Neuroanatomical Correlates of Phonological Processing of Chinese Characters and Alphabetic Words: A Meta-Analysis'. Human Brain Mapping 25 (1): 83-91. https://doi.org/10/dcddf6.

Tenesa, Albert, and Chris S. Haley. 2013. 'The Heritability of Human Disease: Estimation, Uses and Abuses'. Nature Reviews Genetics 14 (2): 139-49. https://doi.org/10/f4nm5q.

The Eurowinter Group. 1997. 'Cold Exposure and Winter Mortality from Ischaemic Heart Disease, Cerebrovascular Disease, Respiratory Disease, and All Causes in Warm and Cold Regions of Europe'. The Lancet 349 (9062): 1347. https://doi.org/doi.org/10.1016/S01406736(96)12338-2. 
Tiokhin, Leonid, Joseph Hackman, Shirajum Munira, Khaleda Jesmin, and Daniel Hruschka. 2019. 'Generalizability Is Not Optional: Insights from a Cross-Cultural Study of Social Discounting'. Royal Society Open Science, 14. https://doi.org/10.1098/rsos.181386.

Tishkoff, S. A., F. A. Reed, F. R. Friedlaender, C. Ehret, A. Ranciaro, A. Froment, J. B. Hirbo, et al. 2009. 'The Genetic Structure and History of Africans and African Americans'. Science 324 (5930): 1035-44. https://doi.org/10.1126/science.1172257.

Tjørve, Kathleen M. C., and Even Tjørve. 2017. 'The Use of Gompertz Models in Growth Analyses, and New Gompertz-Model Approach: An Addition to the Unified-Richards Family'. Edited by Roeland M.H. Merks. PLOS ONE 12 (6): e0178691. https://doi.org/10/gbgttg.

Trahan, Lisa, Karla K Stuebing, Merril K Hiscock, and Jack M Fletcher. 2014. 'The Flynn Effect: A Meta-Analysis'. Psychological Bulletin 140 (5): 1332-60. https://doi.org/10.1037/a0037173.

Tucker-Drob, Elliot M., and Timothy C. Bates. 2016. 'Large Cross-National Differences in Gene × Socioeconomic Status Interaction on Intelligence'. Psychological Science 27 (2): 138-49. https://doi.org/10.1177/0956797615612727.

Tucker-Drob, Elliot M., Daniel A. Briley, and K. Paige Harden. 2013. 'Genetic and Environmental Influences on Cognition Across Development and Context'. Current Directions in Psychological Science 22 (5): 349-55. https://doi.org/10.1177/0963721413485087.

Turkheimer, Eric. 2000. 'Three Laws of Behavior Genetics and What They Mean'. Current Directions in Psychological Science 9 (5): 160-64. https://doi.org/10.1111/1467-8721.00084.

Turkheimer, Eric, Andreana Haley, Mary Waldron, Brian D’Onofrio, and Irving I. Gottesman. 2003. 'Socioeconomic Status Modifies Heritability of IQ in Young Children'. Psychological Science 14 (6): 623-28. https://doi.org/10/bctj6k.

Turkheimer, Eric, Erik Pettersson, and Erin E. Horn. 2014. 'A Phenotypic Null Hypothesis for the Genetics of Personality'. Annual Review of Psychology 65 (1): 515-40. https://doi.org/10.1146/annurev-psych-113011-143752. 
Uchiyama, Ryutaro, and Michael Muthukrishna. in press. 'Cultural Evolutionary Neuroscience'. In Oxford Handbook of Cultural Neuroscience and Global Mental Health. Oxford: Oxford University Press. https://doi.org/10.31234/osf.io/3pj8a.

Ujma, Péter P., Nóra Eszlári, András Millinghoffer, Bence Bruncsics, Péter Petschner, Péter Antal, Bill Deakin, Gerome Breen, György Bagdy, and Gabriella Juhász. 2020. 'Genetic Effects on Educational Attainment in Hungary'. Preprint. bioRxiv. https://doi.org/10.1101/2020.01.13.905034.

UNESCO Institute for Statistics. 2013. Adult and Youth Literacy: National, Regional and Global Trends, 1985-2015. http://uis.unesco.org/sites/default/files/documents/adult-and-youthliteracy-national-regional-and-global-trends-1985-2015-en_0.pdf.

Uskul, Ayse K, Shinobu Kitayama, and Richard E Nisbett. 2008. 'Ecocultural Basis of Cognition: Farmers and Fishermen Are More Holistic than Herders'. Proceedings of the National Academy of Sciences 105 (25): 8552-56. https://doi.org/10.1073/pnas.0803874105.

Van der Wielen, Reggy PJ, LCPGM De Groot, WA Van Staveren, MRH Löwik, H Van den Berg, J Haller, and O Moreiras. 1995. 'Serum Vitamin D Concentrations among Elderly People in Europe'. The Lancet 346 (8969): 207-10.

Visioli, Francesco, and Claudio Galli. 2001. 'The Role of Antioxidants in the Mediterranean Diet'. Lipids 36 (S1): S49-52. https://doi.org/10.1007/s11745-001-0682-z.

Visscher, Peter M., William G. Hill, and Naomi R. Wray. 2008. 'Heritability in the Genomics Era - Concepts and Misconceptions'. Nature Reviews Genetics 9 (4): 255-66. https://doi.org/10.1038/nrg2322.

Visscher, Peter M., Naomi R. Wray, Qian Zhang, Pamela Sklar, Mark I. McCarthy, Matthew A. Brown, and Jian Yang. 2017. '10 Years of GWAS Discovery: Biology, Function, and Translation'. The American Journal of Human Genetics 101 (1): 5-22. https://doi.org/10.1016/j.ajhg.2017.06.005.

Vitzthum, Virginia J. 2003. 'A Number No Greater than the Sum of Its Parts: The Use and Abuse of Heritability'. Human Biology 75 (4): 539-58. https://doi.org/10.1353/hub.2003.0064.

Vygotsky, Lev S. 1980. Mind in Society: The Development of Higher Psychological Processes. Cambridge, MA: Harvard University Press. https://doi.org/10.2307/j.ctvjf9vz4. 
Waddington, C. H. 1953. 'Genetic Assimilation of an Acquired Character'. Evolution 7 (2): 118. https://doi.org/10.2307/2405747.

Wall, Jeffrey D., Eric W. Stawiski, Aakrosh Ratan, Hie Lim Kim, Changhoon Kim, Ravi Gupta, Kushal Suryamohan, et al. 2019. 'The GenomeAsia 100K Project Enables Genetic Discoveries across Asia'. Nature 576 (7785): 106-11. https://doi.org/10.1038/s41586-0191793-z.

Wang, Qi. 2021. 'The Cultural Foundation of Human Memory'. Annual Review of Psychology 72 (1): 151-79. https://doi.org/10.1146/annurev-psych-070920-023638.

Wasserman, Gail A, Xinhua Liu, Nancy J Lolacono, Pam Factor-Litvak, Nada Morina, Aida Musabegovic, Nait Vrenezi, et al. 1997. 'Lead Exposure and Intelligence in 7-Year-Old Children: The Yugoslavia Prospective Study.' Environmental Health Perspectives 105 (9): 7. https://dx.doi.org/10.1289\%2Fehp.97105956.

Waxman, Sandra, Douglas Medin, and Norbert Ross. 2007. 'Folkbiological Reasoning from a Cross-Cultural Developmental Perspective: Early Essentialist Notions Are Shaped by Cultural Beliefs.' Developmental Psychology 43 (2): 294-308. https://doi.org/10.1037/00121649.43.2.294.

Werker, Janet F., and Takao K. Hensch. 2015. 'Critical Periods in Speech Perception: New Directions'. Annual Review of Psychology 66 (1): 173-96. https://doi.org/10.1146/annurevpsych-010814-015104.

White, Cindel, Michael Muthukrishna, and Ara Norenzayan. 2020. 'Worldwide Evidence of Cultural Similarity among Co-Religionists within and across Countries Using the World Values Survey'. PsyArXiv. https://psyarxiv.com/uetg6/.

Whiten, Andrew. 2019. 'Cultural Evolution in Animals'. Annual Review of Ecology, Evolution, and Systematics 50 (1): 27-48. https://doi.org/10.1146/annurev-ecolsys-110218-025040.

Wilmer, Henry H., Lauren E. Sherman, and Jason M. Chein. 2017. 'Smartphones and Cognition: A Review of Research Exploring the Links between Mobile Technology Habits and Cognitive Functioning'. Frontiers in Psychology 8: 605. https://doi.org/10/ggdfkw.

Wilson, David Sloan. 2019. This View of Life: Completing the Darwinian Revolution.

Wingo, A P, L M Almli, J S Stevens, T Jovanovic, T S Wingo, G Tharp, Y Li, et al. 2017. 'Genome-Wide Association Study of Positive Emotion Identifies a Genetic Variant and a 
Role for MicroRNAs'. Molecular Psychiatry 22 (5): 774-83. https://doi.org/10.1038/mp.2016.143.

Wolf, Yuri I., and Eugene V. Koonin. 2013. 'Genome Reduction as the Dominant Mode of Evolution: Prospects \& Overviews'. Bioessays 35 (9): 829-37. https://doi.org/10.1002/bies.201300037.

Wong, Patrick C. M., Xin Kang, Kay H. Y. Wong, Hon-Cheong So, Kwong Wai Choy, and Xiujuan Geng. 2020. 'ASPM-Lexical Tone Association in Speakers of a Tone Language: Direct Evidence for the Genetic-Biasing Hypothesis of Language Evolution'. Science Advances 6 (22): eaba5090. https://doi.org/10/ggxwrb.

Woodley, Michael A. 2012. 'A Life History Model of the Lynn-Flynn Effect'. Personality and Individual Differences 53 (2): 152-56. https://doi.org/10.1016/j.paid.2011.03.028.

'World Bank Group - International Development, Poverty, \& Sustainability'. 2020. World Bank. https://www.worldbank.org/.

Wrangham, Richard. 2017. 'Control of Fire in the Paleolithic: Evaluating the Cooking Hypothesis'. Current Anthropology 58 (S16): S303-13. https://doi.org/10.1086/692113.

Young, Alexander I, Stefania Benonisdottir, Molly Przeworski, and Augustine Kong. 2019. 'Deconstructing the Sources of Genotype-Phenotype Associations in Humans', 6.

Zanden, J van, Joerg Baten, Marco Mira d'Ercole, Auke Rijpma, and Marcel P. Timmer. 2014. How Was Life?: Global Well-Being since 1820. Paris: OECD Publishing. https://doi.org/10.1787/9789264214262-en.

Zeeuw, Eveline L. de, Eco J.C. de Geus, and Dorret I. Boomsma. 2015. 'Meta-Analysis of Twin Studies Highlights the Importance of Genetic Variation in Primary School Educational Achievement'. Trends in Neuroscience and Education 4 (3): 69-76. https://doi.org/10.1016/j.tine.2015.06.001.

Zhang, Xin, Xi Chen, and Xiaobo Zhang. 2018. 'The Impact of Exposure to Air Pollution on Cognitive Performance'. Proceedings of the National Academy of Sciences 115 (37): 9193-97. https://doi.org/10/ctc3.

Zietsch, B. P., R. Kuja-Halkola, H. Walum, and K. J. H. Verweij. 2014. 'Perfect Genetic Correlation between Number of Offspring and Grandoffspring in an Industrialized 
Human Population'. Proceedings of the National Academy of Sciences 111 (3): 1032-36. https://doi.org/10/f24xtm.

Zwir, Igor, Javier Arnedo, Coral Del-Val, Laura Pulkki-Råback, Bettina Konte, Sarah S. Yang, Rocio Romero-Zaliz, et al. 2018. 'Uncovering the Complex Genetics of Human Temperament'. Molecular Psychiatry. https://doi.org/10.1038/s41380-018-0264-5. 